Aus der Abteilung Pädiatrie III - Pädiatrische Kardiologie und Intensivmedizin (Prof. Dr. med. T. Paul)

im Zentrum Kinderheilkunde und Jugendmedizin

der Medizinischen Fakultät der Universität Göttingen

\title{
Biokompatibilitätsuntersuchung von Conduits für den Pulmonalklappenersatz
}

\author{
INAUGURAL-DISSERTATION \\ zur Erlangung des Doktorgrades \\ der Medizinischen Fakultät \\ der Georg-August-Universität zu Göttingen
}

vorgelegt von

Johanna Göbbert

aus

Nienburg/Weser

Göttingen 2010 


\section{Inhaltsverzeichnis}

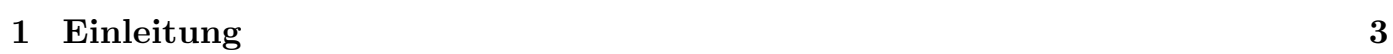

1.1 Angeborene Herzfehler . . . . . . . . . . . . . . . . . . . . . 3

1.2 Therapie bei Pulmonalstenose $\ldots \ldots \ldots \ldots$. . . . . . . . . . . . . . 3

1.3 Pulmonalklappenersatz . . . . . . . . . . . . . . . . . . . . . . . 4

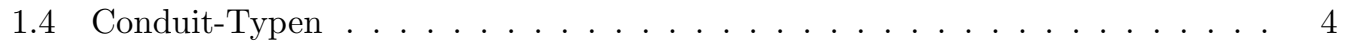

1.5 Biokompatibilität der Conduits $\ldots \ldots \ldots \ldots \ldots \ldots \ldots$

1.6 Zielsetzung der Arbeit $\ldots \ldots \ldots \ldots \ldots$

\begin{tabular}{|lll|}
\hline 2 & Material und Methoden & $\mathbf{7}$
\end{tabular}

2.1 Untersuchte Präparate . . . . . . . . . . . . . . . . . . . . . . . 7

2.2 Präparateaufarbeitung . . . . . . . . . . . . . . . . . . . . . . . . . . . 10

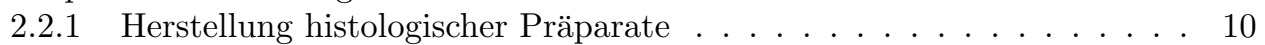

2.2 .2 Immunhistochemie . . . . . . . . . . . . . . . . . . . . . . . 11

2.3 Technische Ausrüstung . . . . . . . . . . . . . . . . . . . . 13

$\begin{array}{llr}3 & \text { Ergebnisse } & 14\end{array}$

$3.1 \quad$ Homografts $\ldots \ldots \ldots \ldots \ldots$. . . . . . . . . . . . . . . . . . . 14

3.1.1 Histopathologische Auswertung des Kontroll-Homografts . . . . . . . . 14

3.1.2 Histopathologische Auswertung der Homograft-Explantate. . . . . . . 15

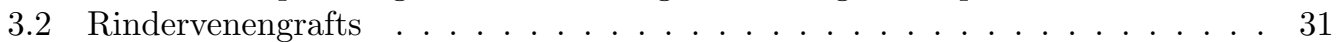

3.2.1 Histopathologische Auswertung der Rindervenengrafts . . . . . . . . . 31

3.3 PTFE-Conduits mit und ohne Schweineklappe . . . . . . . . . . . . . 41

3.3.1 Histopathologische Auswertung der PTFE-Conduits . . . . . . . . . . 41

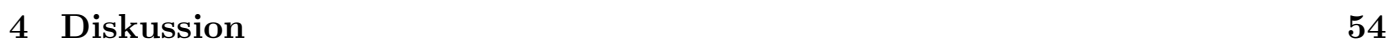

4.1 Methoden . . . . . . . . . . . . . . . . . . . . . . . . . . . 54

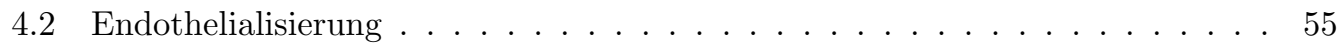

4.3 Pseudointima $\ldots \ldots \ldots \ldots \ldots \ldots$

$4.3 .1 \quad$ Muskelzell-Marker . . . . . . . . . . . . . . . . . . . . . . . . . 58

$4.3 .2 \quad$ Extrazellulärmatrix-Komponenten . . . . . . . . . . . . . . . . . 60

$4.3 .3 \quad$ Entstehungsmechanismen der Pseudointima . . . . . . . . . . . . . . . 60

$4.3 .4 \quad$ Chondroide Metaplasie . . . . . . . . . . . . . . . . . . . . . . . . 63

4.4 Neovaskularisierung und Lakunen . . . . . . . . . . . . . . . . . . . . . . . . 64

4.4 .1 Kleine Gefäße und Kapillaren . . . . . . . . . . . . . . . . . . . . . . 64

4.4 .2 Lakunen . . . . . . . . . . . . . . . . . . . . . . . . . . . . 66

4.5 Entzündungsreaktion . . . . . . . . . . . . . . . . . . . . . . 67

4.5.1 Verwendente Antikörper gegen Oberflächen-Antigene von Entzündungs-

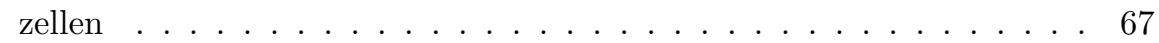

4.5.2 $\quad$ Ergebnisse der immunhistochemischen Charakterisierung der Entzündungs-

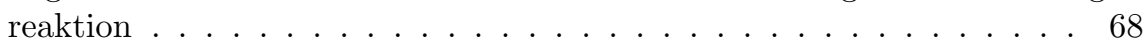

$4.5 .3 \quad$ Entzündungsreaktion bei Conduit-Endokarditis . . . . . . . . . . . 73

4.5.4 Pseudointima-Bildung mit Stenosierung als Manifestation einer Abstoßungsreaktion $\ldots \ldots \ldots \ldots \ldots \ldots \ldots \ldots \ldots \ldots \ldots$

4.6 Thrombenbildung . . . . . . . . . . . . . . . . . . . . . . . . . 74

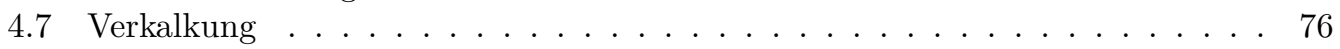


$4.7 .1 \quad$ Verkalkung der Homografts . . . . . . . . . . . . . . . . . . 77

4.7 .2 Verkalkung der Hancock-Conduits und des PTFE-Grafts. . . . . . . . 78

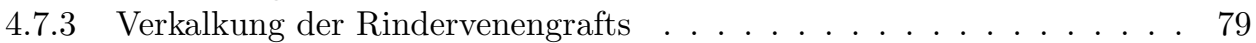

4.7 .4 Mit Verkalkung assoziierte Befunde. . . . . . . . . . . . . . . . . . . . 80

4.8 Taschenklappen $\ldots \ldots \ldots \ldots \ldots$. . . . . . . . . . . . . . . . 80

4.9 Media $\ldots \ldots \ldots \ldots \ldots$. . . . . . . . . . . . . . . . . . . . . 81

4.10 Adventitia . . . . . . . . . . . . . . . . . . . . . . . . . 82

\begin{tabular}{lll}
\hline 5 & Zusammenfassung & 84
\end{tabular}

$\begin{array}{lll}6 & \text { Abkürzungs- und Abbildungsverzeichnis } & 86\end{array}$

\begin{tabular}{lll}
\hline 7 & Literaturverzeichnis & 93
\end{tabular} 


\section{Kapitel 1}

\section{Einleitung}

\subsection{Angeborene Herzfehler}

Herzfehler gehören zu den häufigsten Fehlbildungen bei Neugeborenen. Ihre Inzidenz beträgt circa 8 von 1000 Lebendgeburten. Mindestens $10 \%$ der von kongenitalen Herzfehlern betroffenen Kinder weisen eine Pulmonalstenose auf. Diese kommt als isoliertes Vitium cordis, aber auch gemeinsam mit anderen Defekten vor. Grundsätzlich werden Pulmonalstenosen nach der Lokalisation der Verengung in drei Gruppen unterteilt: Bei der valvulären Pulmonalstenose sind die Klappensegel miteinander verwachsen und/oder verdickt. Des Weiteren kann die Verengung subvalvulär liegen und damit einer Obstruktion des rechtsventrikulären Ausflusstraktes (RVOT) im engeren Sinne entsprechen. Außerdem kann die Stenosierung supravalvulär lokalisiert sein und somit den Pulmonalishauptstamm oder die peripheren Pulmonalarterien betreffen.

Bei 5 bis $10 \%$ aller angeborenen Herzfehler handelt es sich um die Fallot'sche Tetralogie (TOF). Sie ist ein komplexes Vitium cordis und umfasst neben einer Pulmonalstenose einen Ventrikelseptumdefekt (VSD), eine ante- und dextroponierte Aortenwurzel sowie eine aus der Stenosierung resultierende Hypertrophie des rechten Ventrikels. Bei einer Pulmonalatresie besteht keine direkte Verbindung zwischen rechtem Ventrikel und Pulmonalarteriensystem. Das Spektrum der Fehlbildungen reicht von einer angelegten, jedoch vollständig membranös verschlossenen Pulmonalklappe bis zu einem Fehlen von rechtsventrikulärem Ausflusstrakt und Anteilen des Pulmonalarteriensystems.

\subsection{Therapie bei Pulmonalstenose}

Die interventionelle Ballondilatation (Ballonvalvuloplastie, BVP) ist eine Therapieoption bei angeborener valvulärer Pulmonalstenose (Schmaltz et al. 1989). Als Folge kann jedoch eine Pulmonalklappen-Insuffizienz entstehen. Proximal oder distal der Klappe gelegene Stenosen können ebenfalls mittels Ballondilatation behandelt werden (Ballonangioplastie, BAP). Falls eine Dilatation allein keine ausreichende Besserung bewirkt, kann bei diesen beiden Formen per Katheter zusätzlich ein Stent in die Arterie eingesetzt werden. Eine Limitation dieses Therapieverfahrens liegt darin, dass der maximale Stentdurchmesser durch die Größe des Patienten bzw. seiner Gefäße begrenzt ist. Auf Grund des Wachstums des Kindes reicht schließlich der Blutfluss durch den Stent nicht 
mehr aus. Wenn weder eine Dilatation noch eine Stentimplantation zu zufrieden stellenden Ergebnissen führen, müssen eine operative Rekonstruktion oder ein Pulmonalklappenersatz erfolgen.

Seit Ende der 1950er Jahre werden Rekonstruktionen des rechtsventrikulären Ausflusstraktes mit Hilfe von Patches durchgeführt (Lillehei et al. 1986). Da hierdurch in einigen Fällen keine ausreichende Funktion des rechten Herzens hergestellt werden konnte, wurde die Technik weiterentwickelt: Mitte der 1960er Jahre nahmen Rastelli und Kirklin die erste Implantation eines Conduits zwischen rechtem Ventrikel und Pulmonalarteriensystem vor (Rastelli et al. 1965). 1966 setzte Ross erstmals einen klappentragenden Conduit in den rechtsventrikulären Ausflusstrakt eines Menschen ein (Ross und Somerville 1966). In den heute gebräuchlichen Conduits in Pulmonalisposition befindet sich üblicherweise eine Klappe, die in den meisten Fällen aus biologischem Gewebe besteht. Es werden hingegen nur selten mechanische Klappen zum Pulmonalklappenersatz verwandt.

\subsection{Pulmonalklappenersatz}

Der Pulmonalklappenersatz mittels Conduit-Implantation in den rechtsventrikulären Ausflusstrakt ist - wie oben beschrieben - Methode der Wahl bei anders nicht behandelbarer kritischer Pulmonalstenose, aber auch bei Vorliegen einer signifikanten Pulmonalinsuffizienz mit Funktionsbeeinträchtigung des rechten Ventrikels sowie im Fall von Pulmonalatresien, bei denen auf Grund der Ventrikelgröße eine biventrikuläre Korrektur möglich ist. Ein Pulmonalklappenersatz ist allerdings nicht nur indiziert bei Problemen, die primär den rechtsventrikulären Ausflusstrakt, die Pulmonalklappe oder die Pulmonalarterie betreffen. Auch bei folgenden weiteren angeborenen Herzfehlern bestehen Therapieoptionen, die die Implantation eines Conduits in Pulmonalisposition einschließen: Bei einem Truncus arteriosus communis (TAC) münden beide Ventrikel in eine einzige große Arterie. Bei der Korrekturoperation wird die ursprüngliche Truncusklappe zur neuen Aortenklappe, während durch einen Conduit ein neuer rechtsventrikulärer Ausflusstrakt geschaffen wird. Eine therapeutische Möglichkeit bei angeborenen hochgradigen Aortenstenosen ist die sogenannte Ross-Operation. Bei dieser wird die Pulmonalklappe des Patienten nach Resektion der Aortenklappe als Auto-Transplantat in die Aortenposition umgesetzt, der Pulmonalklappenersatz erfolgt durch einen Conduit. Bei einer Transposition der großen Arterien (TGA) mit Ventrikelseptumdefekt und Pulmonalstenose kann eine Rastelli-Operation durchgeführt werden. Hierbei wird der septale Defekt so mit einem Patch verschlossen, dass das Blut fortan aus dem linken Ventrikel in die Aorta fließt. Der Pulmonalarterienstamm wird verschlossen und der rechte Ventrikel über einen Conduit mit dem Pulmonalarteriensystem verbunden.

\subsection{Conduit-Typen}

In der vorliegenden Arbeit wurden drei Typen von RVOT-Conduits untersucht: Bei Homografts handelt es sich um menschliche Aorten- oder Pulmonalisgrafts 
und damit also um Allografts. Sie werden antibiotisch vorbehandelt und kryokonserviert. Des Weiteren können bovine Jugularvenen als Conduit verwendet werden, die unter dem Handelsnamen VenProß) (VenPro Corporation, Irvine, California, USA) bzw. mittlerweile Contegra $\AA$ (Medtronic Incorporation, Minneapolis, Minnesota, USA) vertrieben werden.
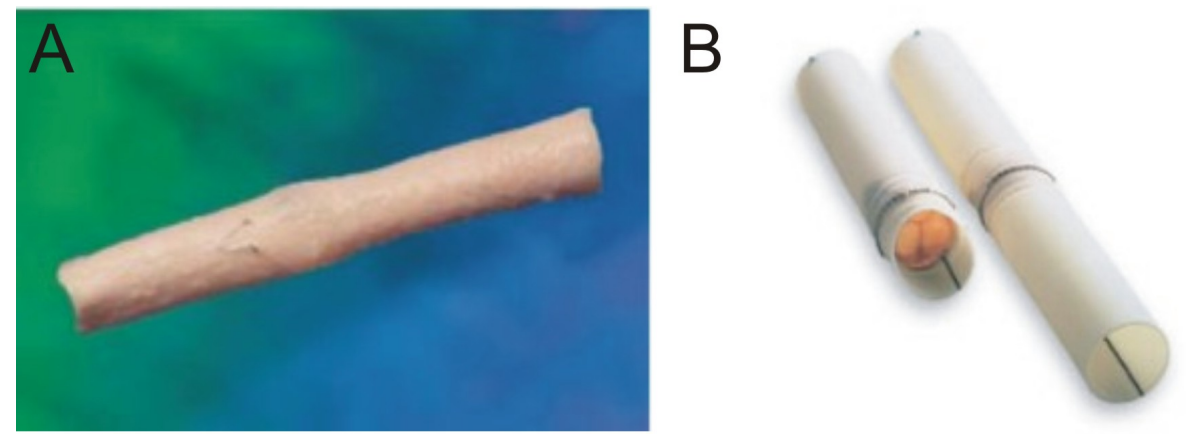

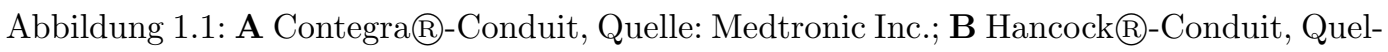
le: Medtronic Inc.

Sie enthalten eine Rinder-Venenklappe als Ersatz für die Pulmonalklappe des Patienten. Diese bovinen Xenografts werden üblicherweise mit Glutaraldehyd vorbehandelt und dadurch avitalisiert und mechanisch stabilisiert. Als weiteres Xenograft kommen porcine Bioprothesen in Frage, die die Bezeichnung Hancock $\AA$ (Medtronic Incorporation, Minneapolis, Minnesota, USA) tragen. Sie bestehen aus einem Schlauch aus Polytetrafluorethylen (PTFE, Handelsname Gore-Tex( $)$, der eine Schweine-Aortenklappe enthält. Deren Klappensegel sind über ein zylinderförmiges Segment aus Schweinegewebe in den PTFE-Schlauch eingenäht. Von außen ist die porcine Klappe zusätzlich durch einen Metallring verstärkt. Auch das Schweine-Gewebe wird vor Implantation durch Glutaraldehyd avitalisiert und stabilisiert. Im Gegensatz zu mechanischen Herzklappen erfordern die beschriebenen Bioprothesen keine lebenslange Antikoagulation.

\subsection{Biokompatibilität der Conduits}

Die derzeit zur Verfügung stehenden Conduits werden durchschnittlich nach zehn bis 15 Jahren ausgetauscht (Brown et al. 2005, Caldarone et al. 2000, Dearani et al. 2003, Lange et al. 2001 und Tweddell et al. 2000). Häufigste Indikation zum Conduit-Wechsel ist eine Conduit-Stenose (Cleveland et al. 1992, Homann et al. 2000 und Stark 1998). Auch eine Insuffizienz der Bioklappen macht oft einen Conduit-Austausch erforderlich.

Im Gegensatz zu klinischen Daten liegen nur wenige histopathologische Informationen zu Gewebereaktionen auf implantierte Conduits vor. Noch seltener sind bisher immunhistochemische Untersuchungen veröffentlicht worden, die sich mit einzelnen Aspekten der Biokompatibilität dieser Implantate befassen (Goffin 1997 und Koolbergen et al. 2002a). 


\subsection{Zielsetzung der Arbeit}

Ziel der vorliegenden Arbeit war es daher, an einer humanen Präparate-Serie nach einem einheitlichen Protokoll die zelluläre und extrazelluläre Gewebereaktion auf die drei beschriebenen Implantattypen zu untersuchen. $\mathrm{Zu}$ diesem Zweck wurden die Conduits zunächst makroskopisch und nach entsprechender Präparation lichtmikroskopisch ausgewertet. Die histopathologische Untersuchung erfolgte sowohl unter Einsatz von konventionellen als auch immunhistochemischen Färbungen. Dabei wurden bei der feingeweblichen Analyse des neugebildeten Gewebes sowie des Conduit-Materials Schwerpunkte insbesondere auf folgende Aspekte gelegt:

- Endothelialisierung

- Muskelzell-Marker

- Extrazellulärmatrix-Komponenten

- Entzündungsreaktion

- Verkalkung

- Thrombenbildung.

Die Resultate der Untersuchungen wurden miteinander wie auch zu geeigneten Daten der Literatur in Beziehung gesetzt. 


\section{Kapitel 2}

\section{Material und Methoden}

\subsection{Untersuchte Präparate}

Im Forschungslabor der Abteilung für pädiatrische Kardiologie und Intensivmedizin der Universitätsmedizin Göttingen werden explantierte kardiovaskuläre Implantate nach einem standardisierten Protokoll histopathologisch aufgearbeitet und auf ihre Biokompatibilität hin untersucht (Sigler et al. 2005). Die Implantate werden im Rahmen von Korrekturoperationen entnommen und zur Analyse eingesandt.

Im Rahmen der vorliegenden Arbeit wurden 24 explantierte Conduits von 22 Patienten untersucht, die jeweils in Pulmonalis-Position implantiert gewesen waren. Es lagen folgende drei Gruppen zur Untersuchung vor: Homografts $(\mathrm{n}=$ 10), Rindervenen-Grafts $(\mathrm{n}=3)$ sowie PTFE-Conduits mit $(\mathrm{n}=10)$ und ohne $(\mathrm{n}=1)$ Schweine-Klappe. Die Conduits wurden im Zeitraum von 1987 bis 2005 implantiert, wobei die Patienten zwischen sieben Tagen und 29 Jahren alt waren. Die Conduits verblieben zwischen sechs Monaten und 19 Jahren und sechs Monaten in ihrer Position im rechtsventrikulären Ausflusstrakt, entsprechend erfolgten die Explantationen in den Jahren 2001 bis 2007. Zusammengefasst in Tabelle 1 finden sich die Diagnosen und vorausgegangenen Interventionen der Patienten, der Implantattyp, das Patientenalter bei Implantation, die Implantationszeiten (Zeitintervalle zwischen Implantation und Explantation) sowie die Gründe für die Explantation. 


\begin{tabular}{|c|c|c|c|c|}
\hline Nr.: & $\begin{array}{l}\text { Diagnosen/ } \\
\text { Inter- } \\
\text { ventionen }\end{array}$ & $\begin{array}{l}\text { Implantat- } \\
\text { typ }\end{array}$ & $\begin{array}{l}\text { Alter bei } \\
\text { Implantation } \\
\text { Implantations- } \\
\text { zeit }\end{array}$ & $\begin{array}{l}\text { Grund } \\
\text { der } \\
\text { Explan- } \\
\text { tation }\end{array}$ \\
\hline 1 & $\begin{array}{l}\text { Subvalv., valv. und supravalv. AoSt; } \\
\text { Z. n. Ross-OP }\end{array}$ & $\begin{array}{l}\text { Ao-HG } \\
19 \mathrm{~mm}\end{array}$ & $\begin{array}{l}\text { 7J } 11 \mathrm{M} \\
8 J\end{array}$ & CSt \\
\hline 2 & $\begin{array}{l}\text { Krit. valv. AoSt; Z. n. Ross-OP; } \\
\text { BAP des HG 8M vor Expl. }\end{array}$ & $\begin{array}{l}\text { Pulm-HG } \\
22 \mathrm{~mm}\end{array}$ & $\begin{array}{l}4 \mathrm{~J} 11 \mathrm{M} \\
10 \mathrm{~J} 8 \mathrm{M}\end{array}$ & CSt \\
\hline 3 & $\begin{array}{l}\text { Krit. valv. AoSt; Z. n. Ross-OP; } \\
\text { Z. n. HG-Austausch; BAP des zweiten } \\
\text { HG 14M vor Expl. }\end{array}$ & $\begin{array}{l}\text { Pulm-HG } \\
24 \mathrm{~mm}\end{array}$ & $\begin{array}{l}15 \mathrm{~J} 7 \mathrm{M} \\
1 J 7 M\end{array}$ & CSt \\
\hline 4 & $\begin{array}{l}\text { TOF; Z. n. Korrektur-OP; } \\
\text { Z. n. Conduit-Austausch } \\
\text { (Hancock gegen Pulm-HG) }\end{array}$ & $\begin{array}{l}\text { Pulm-HG } \\
14 \mathrm{~mm}\end{array}$ & $\begin{array}{l}2 \mathrm{~J} \\
6 M\end{array}$ & CSt \\
\hline 5 & $\begin{array}{l}\text { Noonan-Syndrom mit subvalv. PulmSt; } \\
\text { Z. n. HG-Impl. }\end{array}$ & $\begin{array}{l}\text { Ao-HG } \\
22 \mathrm{~mm}\end{array}$ & $\begin{array}{l}8 \mathrm{~J} 5 \mathrm{M} \\
9 \mathrm{~J} 8 \mathrm{M}\end{array}$ & CSt \\
\hline 6 & $\begin{array}{l}\text { D-TGA, VSD, PulmSt; Z. n. Rastelli- } \\
\text { OP; Z. n. Conduit-Austausch } \\
\text { (Hancock gegen Ao-HG); } \\
\text { rez. bakt. Endokarditiden }\end{array}$ & $\begin{array}{l}\text { Ao-HG } \\
26 \mathrm{~mm}\end{array}$ & $\begin{array}{l}13 \mathrm{~J} 6 \mathrm{M} \\
19 J 6 M\end{array}$ & CSt \\
\hline 7 & $\begin{array}{l}\text { PulmAtresie; Z. n. HG-Impl.; } \\
\text { Stentimpl. 2J 10M vor Expl. } \\
\text { (Jo-Stent } 12 \times 28 \mathrm{~mm} \text { ) }\end{array}$ & $\begin{array}{l}\mathrm{HG} 18 \mathrm{~mm} \\
\text { mit } \\
\text { Stent }\end{array}$ & $\begin{array}{l}11 \mathrm{M} \\
6 J 7 M\end{array}$ & $\begin{array}{l}\text { CSt } \\
\text { CIns }\end{array}$ \\
\hline 8 & $\begin{array}{l}\text { D-TGA, VSD, valv. und subvalv. } \\
\text { PulmSt; Z. n. Rastelli-OP; } \\
\text { BVP 3J 4M vor Expl.; } \\
\text { Stentimpl. 2J 5M vor Expl. } \\
\text { (Jo-Stent } 16 \times 28 \mathrm{~mm} \text { ) }\end{array}$ & $\begin{array}{l}\text { Ao-HG } \\
\text { mit } \\
\text { Stent }\end{array}$ & $\begin{array}{l}5 \mathrm{M} \\
10 J 4 M\end{array}$ & $\begin{array}{l}\text { CSt } \\
\text { CIns }\end{array}$ \\
\hline 9 & $\begin{array}{l}\text { PulmAtresie, VSD; Z. n. HG-Impl.; } \\
\text { Stentimpl. 5J bzw. 2J 2M vor Expl. } \\
\text { (Jo-Stent und covered Jo-Stent } 28 \mathrm{~mm} \text { ) }\end{array}$ & $\begin{array}{l}\text { HG mit } \\
2 \text { Stents }\end{array}$ & $\begin{array}{l}1 \mathrm{~J} 9 \mathrm{M} \\
6 J 2 M\end{array}$ & CSt \\
\hline 10 & Krit. valv. AoSt; Z. n. Ross-OP & $\begin{array}{l}\text { Ao-HG } \\
20 \mathrm{~mm}\end{array}$ & $\begin{array}{l}4 \mathrm{~J} 9 \mathrm{M} \\
10 J 11 M\end{array}$ & CSt \\
\hline 11 & $\begin{array}{l}\text { TAC Typ I; Z. n. Korrektur-OP; } \\
\text { Z.n. Conduit-Austausch (Hancock } \\
\text { gegen RVG); BAP } 1 \mathrm{M} \text { vor Expl. }\end{array}$ & $\begin{array}{l}\text { VenPro } \\
20 \mathrm{~mm}\end{array}$ & $\begin{array}{l}5 \mathrm{~J} 11 \mathrm{M} \\
2 J 2 M\end{array}$ & CSt \\
\hline 12 & $\begin{array}{l}\text { PulmAtresie, VSD; } \\
\text { Z. n. Korrektur-OP }\end{array}$ & $\begin{array}{l}\text { VenPro } \\
12 \mathrm{~mm}\end{array}$ & $\begin{array}{l}1 \mathrm{~W} \\
1 J 8 M 6\end{array}$ & CIns \\
\hline 13 & TOF; Z. n. Korrektur-OP & $\begin{array}{l}\text { VenPro } \\
14 \mathrm{~mm}\end{array}$ & $\begin{array}{l}3 \mathrm{M} 2 \mathrm{~W} \\
5 J 6 M\end{array}$ & $\begin{array}{l}\text { CSt } \\
\text { CIns }\end{array}$ \\
\hline 14 & $\begin{array}{l}\text { TOF; Z. n. Korrektur-OP; } \\
\text { bakt. Endokarditis; } \\
\text { BAP } 1 \mathrm{~W} \text { vor Expl. }\end{array}$ & $\begin{array}{l}\text { Hancock } \\
14 \mathrm{~mm}\end{array}$ & $\begin{array}{l}9 \mathrm{M} 2 \mathrm{~W} \\
1 J 2 M\end{array}$ & $\begin{array}{l}\text { CSt } \\
\text { CIns } \\
\text { bakt. E }\end{array}$ \\
\hline 15 & $\begin{array}{l}\text { PulmAtresie, VSD, } \\
\text { multifokale Lungenperf.; } \\
\text { Z. n. Korrektur-OP }\end{array}$ & $\begin{array}{l}\text { klappenl. } \\
\text { PTFE-C. } \\
15 \mathrm{~mm}\end{array}$ & $\begin{array}{l}3 \mathrm{~J} 3 \mathrm{M} \\
15 \mathrm{~J} 8 \mathrm{M}\end{array}$ & CSt \\
\hline
\end{tabular}




\begin{tabular}{|c|c|c|c|c|}
\hline Nr.: & $\begin{array}{l}\text { Diagnosen/ } \\
\text { Inter- } \\
\text { ventionen }\end{array}$ & $\begin{array}{l}\text { Implantat- } \\
\text { typ }\end{array}$ & $\begin{array}{l}\text { Alter bei } \\
\text { Implantation } \\
\text { Implantations- } \\
\text { zeit }\end{array}$ & $\begin{array}{l}\text { Grund } \\
\text { der } \\
\text { Explan- } \\
\text { tation }\end{array}$ \\
\hline \multirow[t]{2}{*}{16} & TAC Typ I; & Hancock & $5 \mathrm{~W}$ & \multirow[t]{2}{*}{ CSt } \\
\hline & Z. n. Korrektur-OP & $14 \mathrm{~mm}$ & $5 J 6 M$ & \\
\hline \multirow[t]{2}{*}{17} & PulmAtresie, VSD; & Hancock & $1 \mathrm{~J} 5 \mathrm{M}$ & \multirow[t]{2}{*}{ CSt } \\
\hline & Z. n. Korrektur-OP & $16 \mathrm{~mm}$ & $10 J 4 M$ & \\
\hline \multirow[t]{2}{*}{18} & PulmAtresie, VSD, multifokale & \multirow[t]{2}{*}{ Hancock } & $2 \mathrm{~J} 9 \mathrm{M}$ & CSt \\
\hline & Lungenperf.; Z. n. Korrektur-OP & & $\eta J 7 M$ & CIns \\
\hline \multirow[t]{2}{*}{19} & TAC Typ II; & Hancock & $4 \mathrm{~W}$ & CSt \\
\hline & Z. n. Korrektur-OP & $12 \mathrm{~mm}$ & $5 J$ & CIns \\
\hline \multirow[t]{2}{*}{20} & \multirow[t]{2}{*}{ TOF; Z. n. Korrektur-OP } & Hancock & $5 \mathrm{~W}$ & CSt \\
\hline & & $12 \mathrm{~mm}$ & $10 \mathrm{M}$ & CIns \\
\hline \multirow[t]{2}{*}{21} & \multirow{2}{*}{$\begin{array}{l}\text { TOF; Z. n. Korrektur-OP; } \\
\text { BAP 7M vor Expl. }\end{array}$} & Hancock & 2J $1 \mathrm{M}$ & CSt \\
\hline & & $16 \mathrm{~mm}$ & $7 J$ & CIns \\
\hline \multirow[t]{2}{*}{22} & PulmAtresie, VSD, multifokale & Hancock & 5J $11 \mathrm{M}$ & \multirow[t]{2}{*}{ CSt } \\
\hline & $\begin{array}{l}\text { Lungenperf.; Z n. Korrektur-OP; } \\
\text { BAP 1J 9M vor Expl. }\end{array}$ & $20 \mathrm{~mm}$ & $9 J 8 M$ & \\
\hline \multirow[t]{2}{*}{23} & PulmAtresie, VSD, multifokale & Hancock & 2J $9 \mathrm{M}$ & \multirow[t]{2}{*}{ CIns } \\
\hline & Lungenperf.; Z. n. Korrektur-OP & $16 \mathrm{~mm}$ & $1 J 2 M$ & \\
\hline 24 & DORV, AV-Diskordanz, PulmSt; & $\begin{array}{l}\text { Hancock } \\
20 \mathrm{~mm}\end{array}$ & $\begin{array}{l}29 \mathrm{~J} \\
q J g M\end{array}$ & CSt \\
\hline
\end{tabular}

Tabelle 2.1: Übersicht der Präparate. (AoSt Aortenstenose, Ao-HG Aorten-Homograft, CSt Conduit-Stenose, Expl. Explantation, Pulm-HG Pulmonalis-Homograft, PulmSt Pulmonalstenose, Impl. Implantation, PulmAtresie Pulmonalatresie, CIns ConduitKlappeninsuffizienz, RVG Rindervenengraft, bakt. E bakterielle Endokarditis, Lungenperf. Lungenperfusion, klappenl. PTFE-C. klappenloser PTFE-Conduit, DORV Double Outlet Right Ventricle)

Bei den explantierten Conduits 2 und 3 handelte es sich um Homografts, die nacheinander bei demselben Patienten implantiert waren. Bei dem Patienten war eine Ross-Operation durchgeführt worden, die eine Conduit-Implantation im rechtsventrikulären Ausflusstrakt erforderte. Beide Homografts mussten wegen einer Stenose explantiert werden und wurden acht bzw. 14 Monate vorher per BAP dilatiert. Die Implantationszeit des ersten Conduits betrug zehn Jahre und acht Monate. Der zweite war für lediglich ein Jahr und sieben Monate implantiert. Auch von einem weiteren Patienten wurden zwei explantierte Conduits untersucht. Bei dem ersten handelte es sich um ein Hancock@-Graft (14), das nach 14 Monaten wegen Stenosierung, Insuffizienz und einer bakteriellen Endokarditis mit Verengung der distalen Conduit-Anastomose entfernt wurde. Eine Woche zuvor war der Versuch einer Ballonangioplastie unternommen worden. Das daraufhin implantierte Homograft (4) wurde nach nur sechs Monaten wegen Stenosierung wieder explantiert.

Bei einem anderen Patienten wurde im Rahmen eines Aortenklappen-Ersatzes bei darüber hinaus vorliegender Homograft-Stenose im RVOT das stenosierende Material, das im OP-Bericht als Kalk-Spange bezeichnet wurde, reseziert; es erfolgte kein kompletter Homograft-Wechsel. Das entfernte Material wurde als Präparat 5 nach den beschriebenen Kriterien untersucht. Dabei fanden sich auch Anteile der Conduit-Wand.

Das Homograft einer Patientin (6) wurde nach einer Implantationszeit von 19 
Jahren und sechs Monaten auf Grund einer Stenose explantiert. Zuvor waren rezidivierend bakterielle Endokarditiden aufgetreten, die zum Zeitpunkt der Explantation jedoch durch Antibiose klinisch erfolgreich behandelt waren.

Zwei der Homografts $(7,8)$ waren dilatiert und mit einem Stent versehen worden, in ein Homograft (9) waren zwei Stents eingesetzt worden. Unter den Rindervenengrafts war eines einen Monat vor der endgültigen Explantation per BAP aufgedehnt worden (11). Auch die Hancock-Conduits 21 und 22 waren sieben Monate bzw. ein Jahr und neun Monate vor ihrer Entfernung dilatiert worden.

In der dritten Gruppe untersuchter Conduits befand sich als Nummer 15 ein klappenloser PTFE-Conduit. Dieser war für 15 Jahre und acht Monate implantiert gewesen und schließlich wegen Stenosierung entfernt worden.

\subsection{Präparateaufarbeitung}

\subsubsection{Herstellung histologischer Präparate}

Die Conduits wurden nach ihrer chirurgischen Entfernung mit umgebendem Gewebe in Formalin (Phosphat-gepuffert, 4\%) mindestens 48 Stunden lang fixiert und dann zunächst makroskopisch untersucht. Die weitere Aufarbeitung der Conduits war in erster Linie abhängig davon, ob Metall im Präparat enthalten war. Die Präparate ohne Metallanteile wurden in Paraffin eingebettet. Hierfür wurden sie nach Auswaschen des Formalins zunächst in einer aufsteigenden Ethanol-Reihe entwässert, mit Xylol behandelt und in Paraffin eingegossen. Anschließend wurden mit einem Rotationsmikrotom (Leica RM 2165) Schnitte mit einer Stärke von etwa $5 \mu \mathrm{m}$ hergestellt. Bei denjenigen Präparaten mit metallischen Implantatanteilen oder ausgeprägter Verkalkung erfolgte nach Auswaschen des Formalins und Entwässerung in einer aufsteigenden Ethanol-Reihe die Einbettung in das Kunstharz Methylmethacrylat (Technovit 9100, Kulzer und Co., Wehrheim, Deutschland). Nach Aushärtung wurden die Präparatblöcke mit Silikonkleber (ELASTOSIL E41, Wacker Chemie GmbH, München, Deutschland) auf Glas-Objektträgern fixiert. Danach wurden mittels Diamantsäge (300CP, Exakt GmbH, Norderstedt, Deutschland) Scheiben mit einer Stärke von etwa 200 bis $300 \mu \mathrm{m}$ hergestellt. Diese wurden mit einem Mikro-Schleif-System (400CS, Exakt GmbH, Norderstedt, Deutschland) auf eine Dicke von 10 bis $30 \mu \mathrm{m}$ geschliffen.

Folgende konventionellen histologischen Färbungen wurden durchgeführt: Die Färbung nach Richardson wurde bei den Methylmethacrylat-Schliffen angewandt. Sie färbt basophile Strukturen blau und metachromatische Strukturen rotviolett. Die Standardfärbung mit Hämatoxylin-Eosin (HE) erfolgte an den Paraffin-Schnitten. Sie färbt basophile Strukturen blau und basische Strukturen rot. Die Elastica-van-Giesson-Färbung (EvG) färbt elastische Strukturen tiefrot und wurde an den Paraffin-Schnitten eingesetzt. Durch die PikrosiriusRot-Färbung werden in der Fluoreszenz-Mikroskopie die fibrillären Kollagene I und III differenziert. Kollagen-I-Fasern werden als orangefarbene und Kollagen-III-Fasern als grüne Strukturen sichtbar. Sie wurde an mehreren Paraffin-Schnitten durchgeführt. An einigen Präparaten erfolgte eine Färbung 
nach von Kossa, mit der Calcium-Phosphat-Kristalle schwarz angefärbt und somit Kalkablagerungen sichtbar gemacht werden können.

\subsubsection{Immunhistochemie}

Darüber hinaus erfolgten immunhistochemische Färbungen. Um eine Bindung der Primärantikörper an die Ziel-Antigene im histologischen Präparat zu ermöglichen, war das Herauslösen des Paraffins bzw. des Methylmethacrylats nötig. Die Entparaffinierung erfolgte mittels Xylol und einer absteigenden Alkoholreihe. Das Kunstharz wurde mit Xylol, Methoxyethylacetat und Aceton aus den Präparaten herausgelöst. Da hierbei auch Kunststoff-Objektträger angegriffen worden wären, waren an ihrer Stelle Glas-Träger verwendet worden. Um die Antigene freizulegen, wurden die Präparate in einem Dampfgarer entweder mit Citrat-Puffer (pH 6), Tris/EDTA-Puffer (pH 9) bzw. Target Retrieval Solution high pH (Dako Cytomation Denmark A/S, Glostrup, Dänemark) oder Proteinase $\mathrm{K}$ vorbehandelt. Unspezifische Anfärbung wurde vermieden, indem die endogene Peroxidase supprimiert wurde (mittels Peroxidase Blocking Reagent S001; Dako).

Verwendet wurden vier Primärantikörper gegen muskuläre Antigene: Smooth Muscle Actin (SMA) (Antikörper bezogen von Dako) und Smooth Muscle Myosin (SMMy) (Millipore GmbH, Schwalbach, Deutschland) sind kontraktile Proteine in glatten Muskelzellen und Antigene auch schon wenig ausdifferenzierter fibromuskulärer Zellen. Die Antigene Desmin (Progen Biotechnik GmbH, Heidelberg, Deutschland) und Caldesmon heavy chain (h-Caldesmon) (Dako) finden sich an höher differenzierten Muskelzellen. Desmin ist als Intermediärfilament-Protein Bestandteil des Zytoskeletts, Caldesmon beeinflusst als regulatorisches Protein die Kontraktion glatter Muskelzellen. Vimentin (Dako) ist ein Marker für Zellen mesenchymalen Ursprungs und ebenfalls ein Intermediärfilament-Protein. Der entsprechende Antikörper färbt neben fibromuskulären auch inflammatorische und Endothelzellen an. Durch die KollagenIII-Färbung (Acris Antibodies GmbH, Herford, Deutschland) wurden in den Paraffin-Präparaten Kollagen-III-Fasern der Extrazellulärmatrix (EZM) braun gefärbt. Das Protein Fibrin, als wichtige Substanz im Rahmen der Blutgerinnung, kam durch den Antikörper gegen die humane Fibrin-ß-Kette (American Diagnostica GmbH, Pfungstadt, Deutschland) zur Darstellung. Diese Färbung wurde jedoch erst im Verlauf der Untersuchungen zusätzlich angewendet, so dass Schnitte von nur sieben Präparaten einbezogen werden konnten. Mit dem Primärantikörper gegen von-Willebrand-Faktor (Dako) wurden Endothelzellen identifiziert.

Außerdem kamen Antikörper gegen Oberflächenantigene von Entzündungszellen zu deren Charakterisierung zur Anwendung: Markiert wurden CD3Antigene (Antikörper bezogen von Dako) von T-Lymphozyten, teilweise CD4Antigene der T-Helfer-Subpopulation (Biozol Diagnostica Vertrieb GmbH, Eching, Deutschland) bzw. CD8-Antigene der T-Killerzellen (Abcam plc, Cambridge, Großbritannien) sowie CD79-Antigene von B-Lymphozyten. Hierbei wurde anfänglich ein nicht-humanspezifischer Antikörper gegen CD79 (Dako) verwendet, der im Laufe der Untersuchungen zum Teil durch den humanspezifischen Antikörper CD79alpha (Dako) ergänzt wurde, sofern weitere Schnitte 
oder Schliffe der Präparate vorhanden waren. Darüber hinaus wurden in einigen Schnitten CD138-Antigene von Plasmazellen (Dako) sowie durchgehend CD68-Antigene von Monozyten bzw. Makrophagen (Dako) markiert.

Die Inkubation mit Primärantikörpern erfolgte über Nacht bei $4^{\circ} \mathrm{C}$, diejenige mit Sekundärantikörpern üblicherweise über eine Stunde bei Raumtemperatur. Nach Bindung der spezifischen, mit Peroxidase konjugierten Sekundärantikörper oder alternativ des EnVision+ Dual Link Systems HRP (Dako) (Gout Anti-Mouse and Anti-Rabbit Immunglobulin, signalverstärkt) erfolgte die Färbung der Antigen-Antikörper-Komplexe mittels Diaminobenzidin (DAB). Eine Gegenfärbung wurde in der Regel mit Hämalaun durchgeführt. Schließlich wurden die Schnitte bzw. Schliffe eingedeckt. Vorbehandlung und Verdünnung für die verschiedenen Antikörper sind in Tabelle 2 zusammengefasst.

\begin{tabular}{|c|c|c|}
\hline $\begin{array}{l}\text { Antigen- } \\
\text { Demaskierung }\end{array}$ & Primärantikörper & Sekundärantikörper \\
\hline $\begin{array}{l}\text { Citrat-Puffer ( } \mathrm{pH} 6) \text {, } \\
40 \text { Min im Dampfgarer }\end{array}$ & $\begin{array}{l}\text { Mouse Anti-Human Smooth } \\
\text { Muscle Actin Clone } 1 \mathrm{~A} 4 \\
\text { (Dako); verdünnt } 1: 300\end{array}$ & $\begin{array}{l}\text { Rabbit Anti-Mouse Ig (Dako); } \\
\text { verdünnt } 1: 100\end{array}$ \\
\hline $\begin{array}{l}\text { Citrat-Puffer ( } \mathrm{pH} 6) \\
40 \text { Min im Dampfgarer }\end{array}$ & $\begin{array}{l}\text { Mouse Anti-Smooth Muscle Myosin } \\
\text { heavy chain Clone ID 8 (Millipore); } \\
\text { MM verdünnt } 1: 100 \text {, P 1:500 }\end{array}$ & $\begin{array}{l}\text { Goat Anti-Mouse and Anti-Rabbit Ig, } \\
\text { signalverstärkt (System EnVision, Dako) }\end{array}$ \\
\hline $\begin{array}{l}\text { Tris/EDTA (pH 9) oder } \\
\text { Target Retrieval } \\
\text { Solution high pH, } \\
20 \text { Min im Dampfgarer }\end{array}$ & $\begin{array}{l}\text { Monoclonal Mouse Anti-Human Desmin } \\
\text { Clone D9 (Progen); } \\
\text { verdünnt } 1: 50\end{array}$ & $\begin{array}{l}\text { MM: Goat Anti-Mouse and Anti-Rabbit Ig, } \\
\text { signalverstärkt (System EnVision, Dako); } \\
\text { P: Rabbit Anti-Mouse Ig (Dako), } \\
\text { verdünnt } 1: 100\end{array}$ \\
\hline $\begin{array}{l}\text { Proteinase } \mathrm{K} \text { (Dako), } \\
\text { verdünnt } 1: 500, \\
5 \text { Min bei } 37^{\circ} \mathrm{C}, \\
\text { dann Citrat-Puffer ( } \mathrm{pH} 6) \\
40 \text { Min im Dampfgarer }\end{array}$ & $\begin{array}{l}\text { Monoclonal Mouse Anti-Human } \\
\text { Caldesmon Clone h-CD (Dako); } \\
\text { MM verdünnt } 1: 100 \text {, P } 1: 400\end{array}$ & $\begin{array}{l}\text { Goat Anti-Mouse and Anti-Rabbit Ig, } \\
\text { signalverstärkt (System EnVision, Dako) }\end{array}$ \\
\hline $\begin{array}{l}\text { Citrat-Puffer ( } \mathrm{pH} \mathrm{6} \text { ), } \\
40 \text { Min im Dampfgarer }\end{array}$ & $\begin{array}{l}\text { Monoclonal Mouse Anti-Vimentin } \\
\text { Clone V9 (Dako); } \\
\text { verdünnt } 1: 100\end{array}$ & $\begin{array}{l}\text { MM: Goat Anti-Mouse and Anti-Rabbit Ig, } \\
\text { signalverstärkt (System EnVision, Dako); } \\
\text { P: Rabbit Anti-Mouse Ig (Dako), } \\
\text { verdünnt } 1: 100\end{array}$ \\
\hline $\begin{array}{l}\text { Citrat-Puffer ( } \mathrm{pH} 6) \\
40 \text { Min im Dampfgarer }\end{array}$ & $\begin{array}{l}\text { Monoclonal Mouse Anti-Kollagen-III } \\
\text { (Acris); verdünnt 1: } 1000\end{array}$ & $\begin{array}{l}\text { Rabbit Anti-Mouse Ig (Dako); } \\
\text { verdünnt 1: } 100\end{array}$ \\
\hline $\begin{array}{l}\text { Citrat-Puffer ( } \mathrm{pH} 6) \\
40 \text { Min im Dampfgarer }\end{array}$ & $\begin{array}{l}\text { Monoclonal Mouse Anti-Human Fibrin } \\
\text { B-chain (Am. Diagn.); } \\
\text { verdünnt 1:500 }\end{array}$ & $\begin{array}{l}\text { Rabbit Anti-Mouse Ig (Dako); } \\
\text { verdünnt 1: } 100\end{array}$ \\
\hline $\begin{array}{l}\text { Citrat-Puffer ( } \mathrm{pH} \mathrm{6)} \text {, } \\
40 \text { Min im Dampfgarer }\end{array}$ & $\begin{array}{l}\text { Polyclonal Rabbit Anti-Human } \\
\text { von-Willebrand-Factor (Dako); } \\
\text { verdünnt } 1: 400\end{array}$ & $\begin{array}{l}\text { Swine Anti-Rabbit Ig (Dako); } \\
\text { verdünnt } 1: 100\end{array}$ \\
\hline $\begin{array}{l}\text { Citrat-Puffer ( } \mathrm{pH} 6) \\
\text { 40 Min im Dampfgarer }\end{array}$ & $\begin{array}{l}\text { Polyclonal Rabbit Anti-Human CD3 } \\
\text { (Dako); verdünnt } 1: 100\end{array}$ & $\begin{array}{l}\text { Goat Anti-Mouse and Anti-Rabbit Ig, } \\
\text { signalverstärkt (System EnVision, Dako) }\end{array}$ \\
\hline $\begin{array}{l}\text { Tris/EDTA (pH 9) oder } \\
\text { Target Retrieval } \\
\text { Solution high pH, } \\
20 \text { Min im Dampfgarer }\end{array}$ & $\begin{array}{l}\text { Mouse Anti Human CD4 IgG1 } \\
\text { (Biozol); verdünnt 1:50 }\end{array}$ & $\begin{array}{l}\text { Goat Anti-Mouse and Anti-Rabbit Ig, } \\
\text { signalverstärkt (System EnVision, Dako) }\end{array}$ \\
\hline $\begin{array}{l}\text { Tris/EDTA ( } \mathrm{pH} 9 \text { ) oder } \\
\text { Target Retrieval } \\
\text { Solution high pH, } \\
20 \text { Min im Dampfgarer }\end{array}$ & $\begin{array}{l}\text { Polyclonal Rabbit Anti CD8 } \\
\text { (Abcam); verdünnt 1:75 }\end{array}$ & $\begin{array}{l}\text { Goat Anti-Mouse and Anti-Rabbit Ig, } \\
\text { signalverstärkt (System EnVision, Dako) }\end{array}$ \\
\hline $\begin{array}{l}\text { Tris/EDTA (pH 9), } \\
20 \text { Min im Dampfgarer }\end{array}$ & $\begin{array}{l}\text { Monoclonal Mouse Anti-Human } \\
\text { CD68 Clone PG-M1 (Dako); } \\
\text { verdünt } 1: 200\end{array}$ & $\begin{array}{l}\text { Rabbit Anti-Mouse Immunoglobulin (Dako) } \\
\text { verdünnt } 1: 100\end{array}$ \\
\hline $\begin{array}{l}\text { Tris/EDTA ( } \mathrm{pH} 9 \text { ), } \\
20 \text { Min im Dampfgarer }\end{array}$ & $\begin{array}{l}\text { Monoclonal Mouse Anti-Human } \\
\text { CD79acy Clone HM57 (Dako); } \\
\text { verdünnt } 1: 100\end{array}$ & $\begin{array}{l}\text { Goat Anti-Mouse and Anti-Rabbit Ig, } \\
\text { signalverstärkt (System EnVision, Dako) }\end{array}$ \\
\hline $\begin{array}{l}\text { Tris/EDTA (pH 9), } \\
20 \text { Min im Dampfgarer }\end{array}$ & $\begin{array}{l}\text { Monoclonal Mouse Anti-Human } \\
\text { CD79alpha Clone JCB } 117 \text { (Dako); } \\
\text { verdünnt } 1: 100\end{array}$ & $\begin{array}{l}\text { Rabbit Anti-Mouse Ig (Dako); } \\
\text { verdünnt 1: } 100\end{array}$ \\
\hline $\begin{array}{l}\text { Tris/EDTA (pH 9), } \\
20 \text { Min im Dampfgarer }\end{array}$ & $\begin{array}{l}\text { Monoclonal Mouse Anti-Human } \\
\text { CD138 Clone MI15 (Dako); } \\
\text { verdünnt } 1: 100\end{array}$ & $\begin{array}{l}\text { Goat Anti-Mouse and Anti-Rabbit Ig, } \\
\text { signalverstärkt (System EnVision, Dako) }\end{array}$ \\
\hline
\end{tabular}

Tabelle 2.2: Übersicht der Antikörper. (Ig Immunglobulin, MM Methylmethacrylat, P Paraffin) 


\subsection{Technische Ausrüstung}

Die histologische Auswertung erfolgte mit dem Lichtmikroskop LEICA DM LB (Leica Microsystems GmbH, Wetzlar, Deutschland). Zur Bilddokumentation wurden die CCD-Farbkamera Color View II sowie die Software Analysis 3.2 der Firma Olympus (Olympus Europa Holding GmbH, Hamburg, Deutschland) verwendet. 


\section{Kapitel 3}

\section{Ergebnisse}

Der Ergebnis-Teil dieser Arbeit ist wie folgt strukturiert: Unterteilt nach Conduit-Typen werden die Schichten der Präparate von innen nach außen beschrieben. Angrenzend an das Lumen befand sich üblicherweise eine Lage neu gebildeten Gewebes, die in Anlehnung an die Nomenklatur nativer Gefäße als Pseudointima bezeichnet wurde. Als nächstes folgte das Graft-Gewebe, das hauptsächlich dem ursprünglich implantierten Conduit entsprach. Bei dem umgebenden Gewebe außerhalb der Gefäß-Media des Grafts bzw. des PTFEMaterials handelte es sich um die Adventitia. Außerdem werden - sofern vorhanden - die Klappensegel sowie die implantierten Stents mit umgebendem Gewebe beschrieben.

\section{$3.1 \quad$ Homografts}

Untersucht wurden drei Pulmonalis- und fünf Aorten-Homografts sowie zwei Homografts, deren native Position im Spender unbekannt war. Die Implantationszeiten lagen zwischen sechs Monaten und neunzehneinhalb Jahren. Bei allen Patienten bestand eine Stenosierung des Conduits sowie bei zweien zusätzlich eine Insuffizienz $\mathrm{II}^{\circ}$.

\subsubsection{Histopathologische Auswertung des Kontroll-Homografts}

Als Vergleichsmaterial für die explantierten Homografts diente ein Abschnitt eines Homografts ohne Klappe, das der gleichen Vorbehandlung unterzogen, anschließend jedoch nicht implantiert worden war. Die histopathologische Aufbereitung wurde entsprechend den explantierten Homografts durchgeführt. Das Kontroll-Homograft gliederte sich in drei Schichten. Die schmale lumenwärtige Intima enthielt dichte Fasern, die vielfach positiv für Kollagen III färbten (Abb. 3.1A). Zwischen den Fasern befanden sich längliche sowie stellenweise auch runde Zellen. Die länglichen Zellen färbten positiv für Vimentin, SMA, SMMy, h-Caldesmon und Desmin. Auf der Intima des Homografts zeigte sich lumenseitig eine durchgehende von-Willebrand-Faktor-positive dünne Endothelschicht (Abb. 3.1B). Charakteristisch war die Gefäß-Media, die aus stark gewellten elastischen Fasern (Abb 3.2) sowie Kollagen-III-Fasern bestand. Zwischen den Fasern fanden sich viele spindelförmige Zellen mit ovalem bis länglich-ovalem Zellkern. Sie färbten positiv für SMA, SMMy, Vimen- 
tin, h-Caldesmon und Desmin. Die Adventitia bestand aus lockeren gewellten Kollagen-III-Fasern sowie wenigen, feinen elastischen Fasern. Zwischen den Fasern lagen wenige spindelförmige Zellen, die teilweise Vimentin-positiv waren. Außerdem enthielt die Adventitia dünne Muskelfaser-Bündel, Lipidzellen sowie kleine Vasa vasorum. Die Wände dieser Kapillaren enthielten zum Teil elastische Fasern und ihre Zellen färbten positiv für SMA, Vimentin, SMMy und h-Caldesmon. Zum Lumen der kleinen Gefäße gewandt befand sich eine von-Willebrand-Faktor-positive Zelllage (Abb. 3.1B).
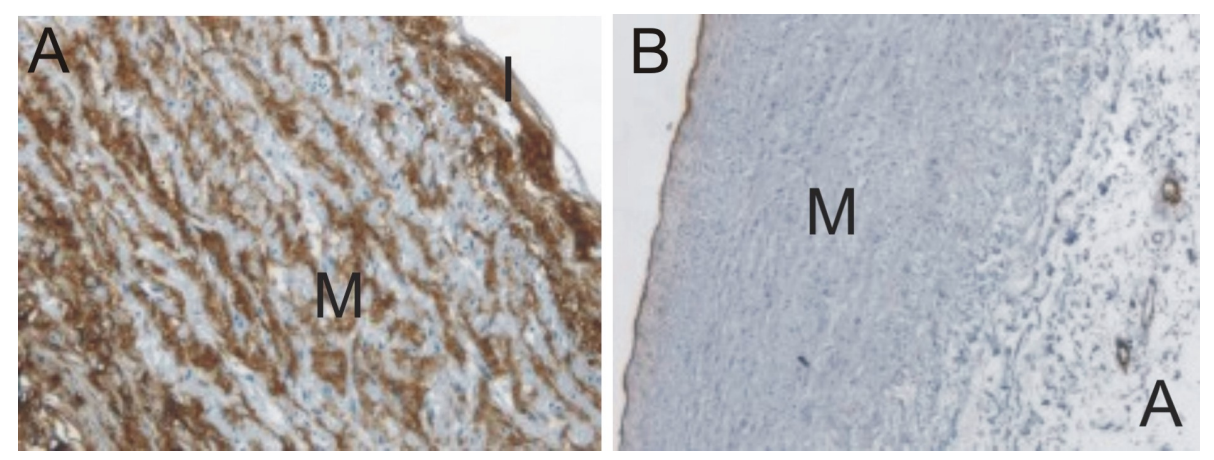

Abbildung 3.1: A positive Färbung (braun) für Kollagen-III-Fasern in Intima (I) und Media (M); Vergrößerung 200fach; Kontroll-Homograft; B von-Willebrand-Faktor-positive Zellen lumenseitig auf der Intima sowie in kleinen Gefäßen der Adventitia; Vergrößerung 100fach; Kontroll-Homograft

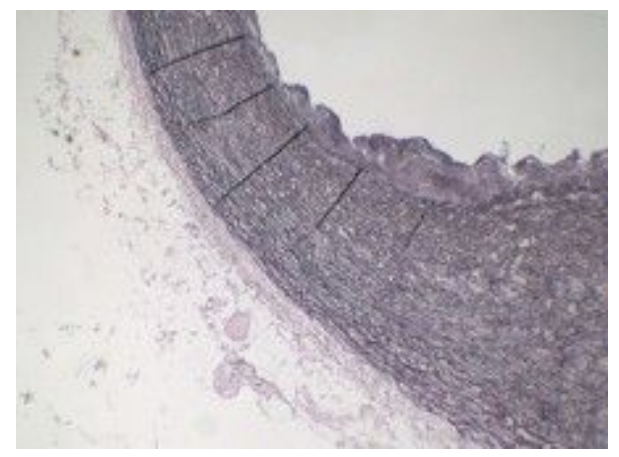

Abbildung 3.2: stark gewellte elastische Fasern der Media; EvG; Vergrößerung 400fach; Kontroll-Homograft

\subsubsection{Histopathologische Auswertung der Homograft-Explantate}

\section{Pseudointima und Endothel}

Alle untersuchten explantierten Homografts wiesen lumenseitig PseudointimaGewebe auf. Die Stärke der Pseudointima war sowohl zirkulär in einer Ebene als auch longitudinal im Verlauf der Conduits unterschiedlich. Histologisch war die Pseudointima in allen Präparaten geprägt durch parallele, häufig leicht gewellte, lange, spindelförmige Zellen, die damit in ihrer Form Fibroblasten ähnelten. Sie waren von Extrazellulärmatrix umgeben und färbten in den immunhistochemischen Färbungen meist positiv für SMA und SMMy (Abb. 
3.3A), vielfach auch für h-Caldesmon und Desmin (Abb. 3.3B) und hatten somit Muskelzell-Eigenschaften. Daher wurden sie in dieser Arbeit als fibromuskuläre Zellen bezeichnet. Die Zellen färbten zudem meist positiv für Vimentin, einen Marker für Zellen mesenchymalen Usrpungs.
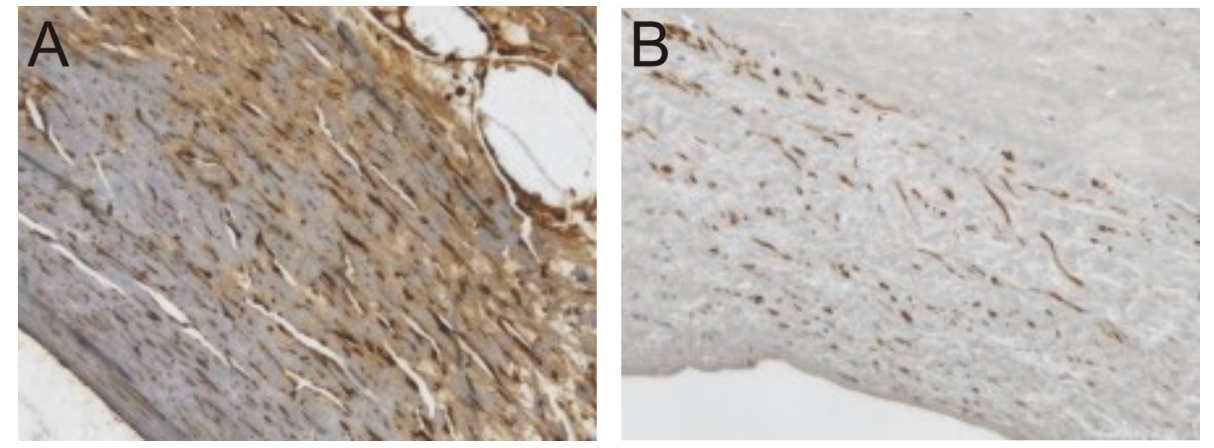

Abbildung 3.3: A SMMy-positive fibromuskuläre Zellen der Pseudointima; Vergrößerung 400fach; Präparat 6; B Desmin-positive fibromuskuläre Zellen der Pseudointima; Vergrößerung 200fach; Präparat 2

Die Extrazellulärmatrix der Pseudointima bestand aus feinen elastischen Fasern, Kollagenfasern und amorpher (zellkernloser) Substanz. Im Vergleich überwogen in der Pikrosirius-Rot-Färbung Kollagenfasern des Subtyps III gegenüber Kollagen-I-Fasern. In den gestenteten Conduits waren die MetallStreben der Stents vielfach von Pseudointima-Gewebe umgeben. Lumenseitig ließ sich in allen Präparaten eine einzellige Endothelschicht erkennen, die positiv für von-Willebrand-Faktor und meist auch für Vimentin färbte (Abb. 3.4) In einigen Präparaten war die Pseudointima-Schicht der Conduit-Wand nicht zirkulär durchgehend. Trotzdem zeigte sich auch hier lumenseitig eine von-Willebrand-Faktor-positive Zellschicht.

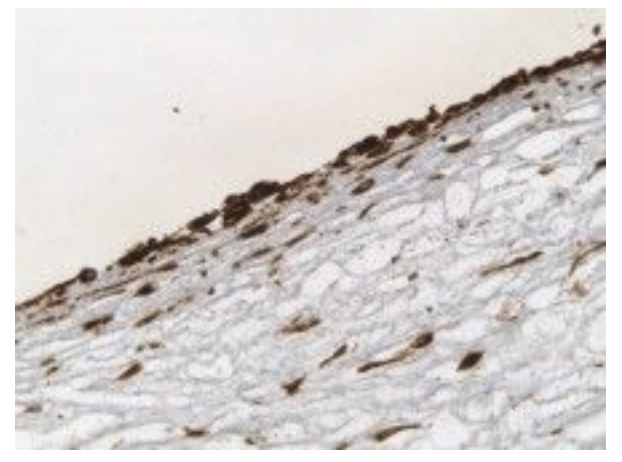

Abbildung 3.4: lumenseitig Vimentin-positive Endothelzellen; Vergrößerung 400fach; Präparat 4

In mehreren Präparaten bestand die Pseudointima stellenweise aus bindegewebigen Septen, zwischen denen sich Zellen befanden (Abb. 3.5). Im Präparat 4 konnten die Septen lumenseitig teilweise als Fibrinfäden identifiziert werden. Eingelagert in dieses Fibringeflecht fanden sich überwiegend Entzündungszellen wie Granulozyten. 

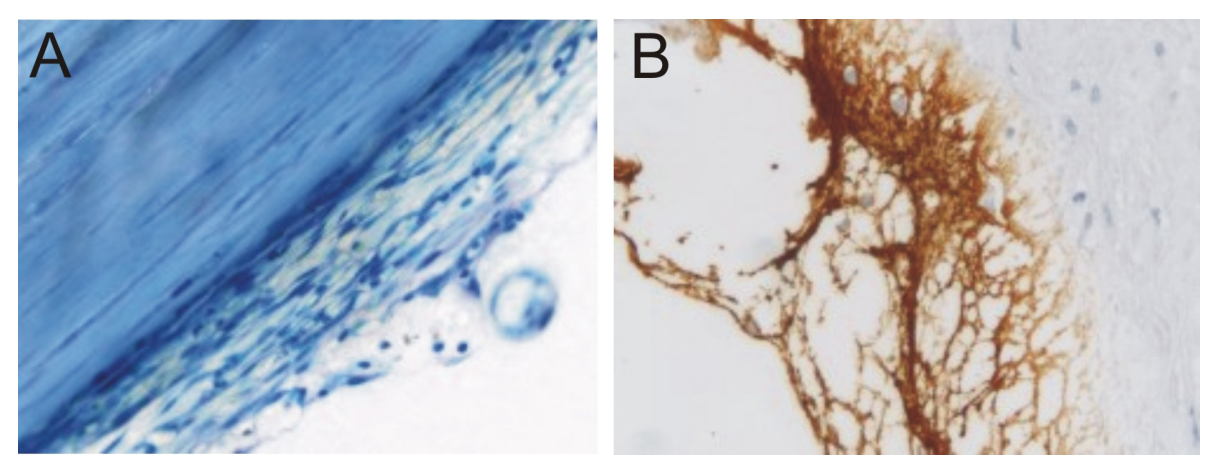

Abbildung 3.5: A septierte Pseudointima; Färbung nach Richardson; Vergrößerung 200fach; Präparat 8; B Entzündungszellen in den Fibrinfäden der Pseudointima; Fibrinfärbung Vergrößerung 400fach; Präparat 4

Nahe der Klappe befanden sich zirkulär in einer Ebene neben Bereichen mit ausgeprägter zellulär organisierter Pseudointima auch Abschnitte mit dünnen Septen (Abb. 3.6A). In den Winkeln zwischen zirkulärer Wand und Klappensegel war das septierte Gewebe sehr deutlich ausgeprägt und enthielt viele inflammatorische Zellen (Abb. 3.6B).
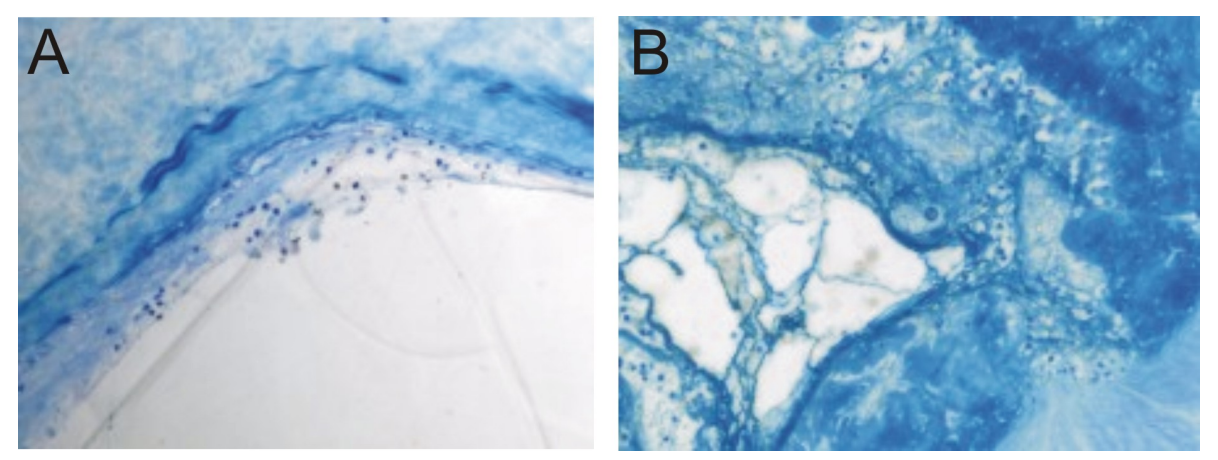

Abbildung 3.6: A Übergang eines bindegewebig organisierten (links unten im Bild) zu einem septierten Abschnitt der Pseudointima; Färbung nach Richardson; Vergrößerung 200fach; Präparat 4; B septierte Pseudointima im Klappen-Winkel; Färbung nach Richardson; Vergrößerung 200fach; Präparat 4

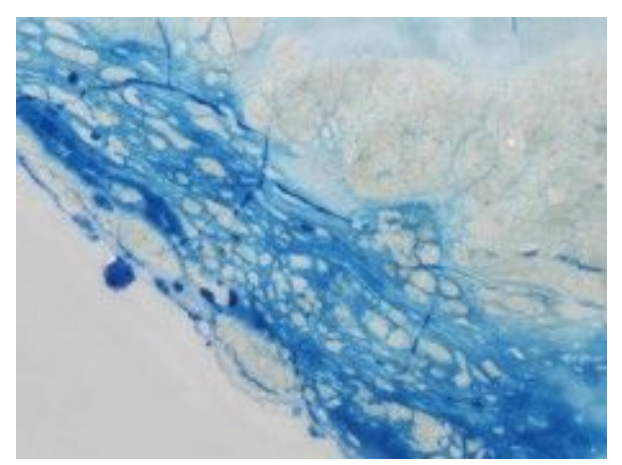

Abbildung 3.7: septierte Pseudointima an der Klappenbasis; Färbung nach Richardson; Vergrößerung 400fach; Präparat 3 
Im weiteren longitudinalen Verlauf dieses Conduits war dessen Wand nicht gleichmäßig zylindrisch konfiguriert, sondern sie war eingefaltet. Besonders in den Falten des Gefäß-Lumens bestand die Pseudointima wiederum zum Teil aus Fibrinsepten und enthielt stellenweise vermehrt Entzündungszellen. Auch das Präparat 3 wies an der Basis der Klappensegel Gewebe auf, das septiert war und zahlreiche Entzündungszellen sowie stellenweise Kapillaren und Erythrozyten enthielt (Abb. 3.7).

Generell ließen sich Kapillaren in der Pseudointima der Conduits erkennen. Sie wiesen lumenseitig meist eine dünne Lage von-Willebrand-Faktor-positiver Zellen auf. Der Endothelschicht nach außen direkt benachbart fanden sich unterschiedlich viele Schichten spindelförmiger Zellen. Diese zeigten sich zirkulär um Gefäß-Querschnitte bzw. im Längsverlauf um die Kapillaren, wenn sie longitudinal angeschnitten waren. Diese Gefäßwände färbten je nach Ausprägung und Erhalt der Schnitte und Schliffe positiv für SMA, SMMy und Vimentin und enthielten teilweise feine elastische Laminae.

Darüber hinaus waren in der Mehrzahl der untersuchten Präparate weitere Hohlräume zu sehen, die lumenwärts ebenfalls meist eine von-WillebrandFaktor-positive Zellschicht aufwiesen. Die umgebenden spindelförmigen Zellen fehlten jedoch. Die Durchmesser der Lumina lagen teilweise deutlich über denen der Kapillaren. Die beschriebenen Hohlräume wurden in dieser Arbeit als Lakunen bezeichnet. In der Pseudointima des Präparats 5 waren die Lakunen beispielsweies zahlreich vorhanden (Abb. 3.8). Sie wirkten zum Teil verzweigt. Kapillaren fanden sich hier dagegen nur sehr vereinzelt in der Nähe des Homograft-Gewebes.

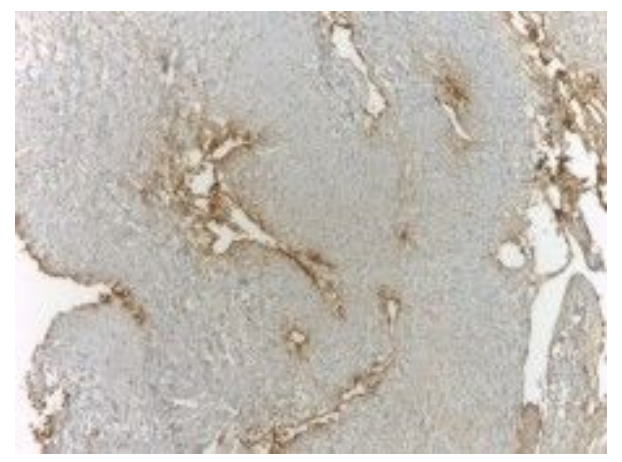

Abbildung 3.8: verzweigte Lakunen in der Pseudointima; von-Willebrand-Färbung; Vergrößerung 100fach; Präparat 5

Acht der zehn untersuchten Präparate zeigten Kalkeinschlüsse, deren Ausprägung sehr unterschiedlich war. Bei allen betroffenen Explantaten waren die Verkalkungen schon makroskopisch zu erkennen. Das Präparat mit der längsten Implantationszeit, Nummer 6, war nach 19 Jahren und sechs Monaten erheblich verkalkt und wies in der haptischen Untersuchung eine zirkulär porzellanartige Konsistenz auf (Abb. 3.9). Entsprechend dieses makroskopischen Befundes waren auch in der mikroskopischen Untersuchung Kalkherde in der Pseudointima und dem Homograft-Gewebe erkennbar (Abb. 3.10). 
Im Präparat 4 zeigten sich in der Pseudointima intrazelluläre Kalk-Einlagerungen. Bei dem Präparat 5 handelte es sich lediglich um die entfernte Kalk-Spange aus einer stenosierten Homograft-Anastomose. Bei der Entnahme dieses Explantates war das eigentliche Homograft in situ belassen worden. Die Pseudointima enthielt ein ausgedehntes Areal mit intra- und extrazellulärer Verkalkung; Zellen und Fasern waren noch erkennbar (Abb. 3.11).

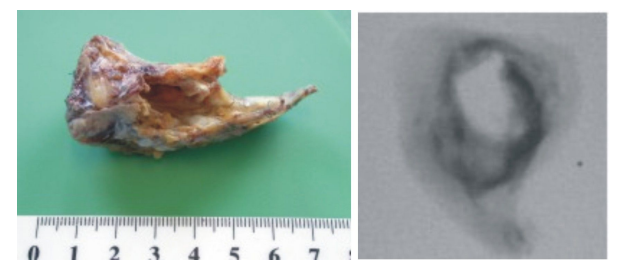

Abbildung 3.9: porzellanartige Verkalkung; anterograde Durchleuchtung des Präparats; Präparat 6

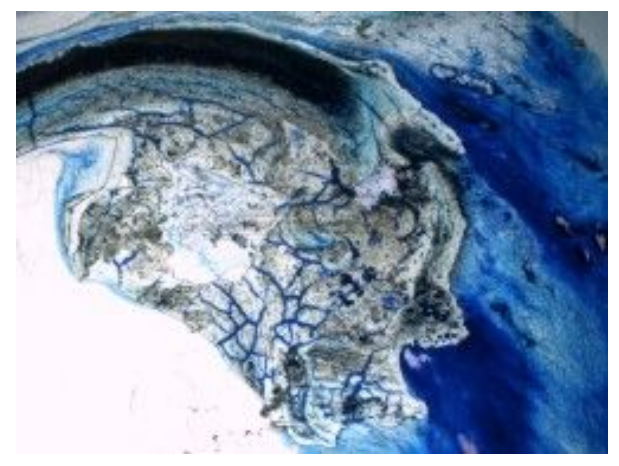

Abbildung 3.10: Kalkareal in der Pseudointima; Färbung nach Richardson; Vergrößerung 16fach; Präparat 6

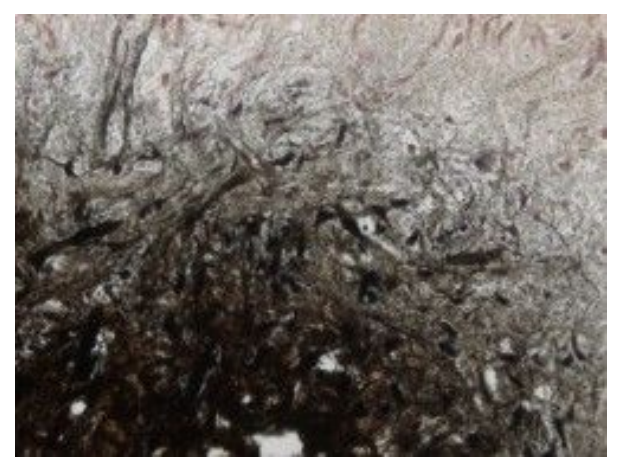

Abbildung 3.11: intra- und extrazelluläre Verkalkung (schwarz) in der Pseudointima; Färbung nach von Kossa; Vergrößerung 400fach; Präparat 5

In den Präparaten 1 (Implantationszeit 5J 8M) und 2 (Implantationszeit 4J 11M) ähnelte sich das histologische Bild: Es fanden sich ausgedehnte Kalkeinschlüsse in der Pseudointima, vor allem nahe der Klappenabgänge, die von fibromuskulären Zellen und Extrazellulärmatrix umgeben waren. Im Präparat 1 befand sich eines der Klappensegel direkt an einem Kalkareal und war gemeinsam mit diesem von Pseudointima-Gewebe bedeckt (Abb. 3.12A). In dem 
Verkalkungsherd fanden sich Mikro-Einblutungen in Form von ErythrozytenAnsammlungen sowie Infiltrate von Entzündungszellen (Abb. 3.12B).
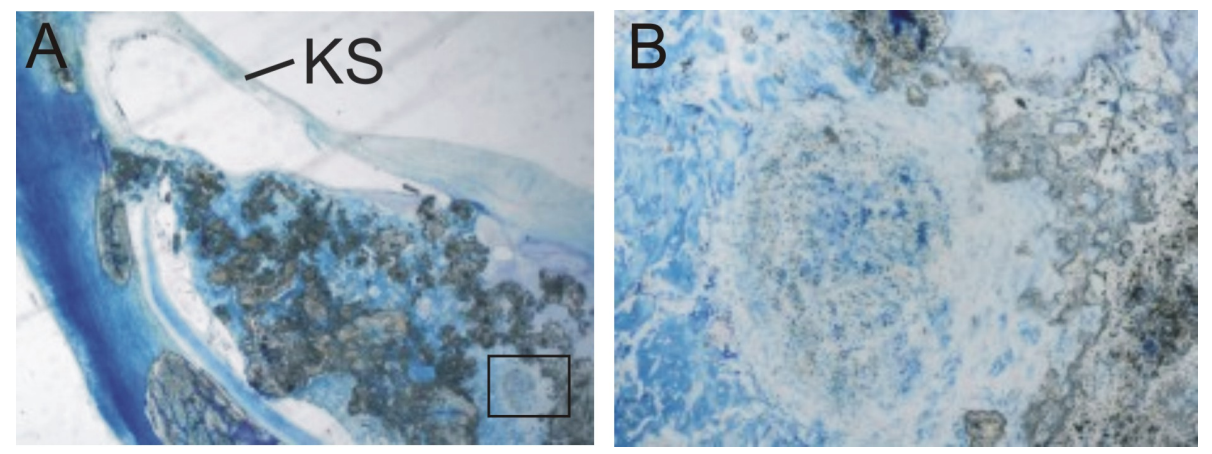

Abbildung 3.12: A Klappensegel (KS) an dem Kalkareal in der Pseudointima; Färbung nach Richardson; Vergrößerung 16fach; Präparat 1; B Entzündungszellen und Erythrozyten innerhalb des Kalkareals am Klappensegel; Ausschnitt aus A; Färbung nach Richardson; Vergrößerung 100fach; Präparat 1

In den Präparaten 1 und 2 befanden sich in der Färbung nach Richardson in der Pseudointima umgeben von amorpher Matrix wabig angeordnete Zellen mit runden Zellkernen (Abb. 3.13). Somit bot sich das Bild einer chondroiden Metaplasie.
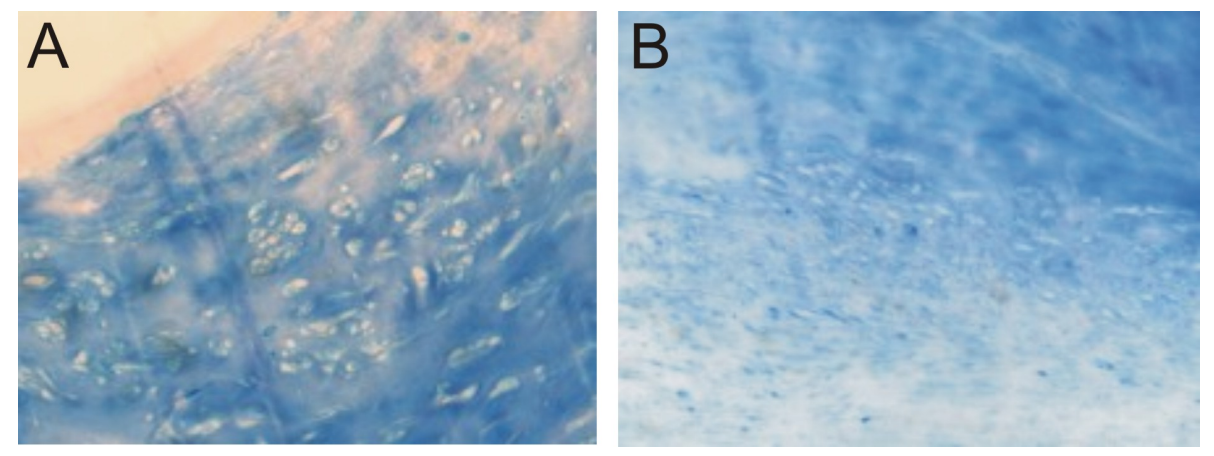

Abbildung 3.13: A chondroide Metaplasie in der Pseudointima; Färbung nach Richardson; Vergrößerung 400fach; Präparat 1; B chondroide Metaplasie in der Pseudointima; Färbung nach Richardson; Vergrößerung 200fach; Präparat 2

In allen untersuchten Homografts enthielt die Pseudointima Entzündungszellen wie Granulozyten, Lymphozyten und Makrophagen. Mit einer immunhistochemischen Färbung mit Antikörpern gegen CD3 ließen sich in allen Präparaten T-Lymphozyten in der Pseudointima nachweisen. Sie lagen einzeln. In den Präparaten 2, 3, 7 und 10 waren sie vergleichsweise zahlreich. Ein Teil dieser Lymphozyten war CD8-positiv. In der Pseudointima aller Präparate befanden sich CD68-positive Makrophagen. Auch diese lagen einzeln. Relativ viele Histiozyten enthielt die Pseudointima in den Präparaten 3 und 6, wo sie vor allem lumennah zu finden waren. Mit dem Antikörper gegen CD79alpha ließen sich im Präparat 4 einige einzeln liegende B-Lymphozyten in der Pseudointima und am Übergang zum Homograft-Gewebe nachweisen. In der Pseu- 
dointima von vier weiteren Präparaten zeigten sich einzelne CD79-positive BLymphozyten bei Färbung mit dem nicht-human-spezifischen CD79-Antikörper. CD138-positive Plasma-Zellen waren in der Pseudointima mit dem verwandten Antikörper nicht nachweisbar.

In den Falten des Gefäßlumens im Präparat 4 enthielt die dort septierte Pseudointima neben einigen CD79alpha-positiven B-Zellen auch CD3- und einige CD8-positive T-Lymphozyten sowie CD68-positive Makrophagen, die wiederum jeweils einzeln lagen (Abb. 3.14). Die T-Zellen befanden sich innerhalb der Pseudointima in der Regel lumenseitig. Auch im septierten Material in den Winkeln am Klappenabgang zeigten sich viele Granulozyten und Lymphozyten.

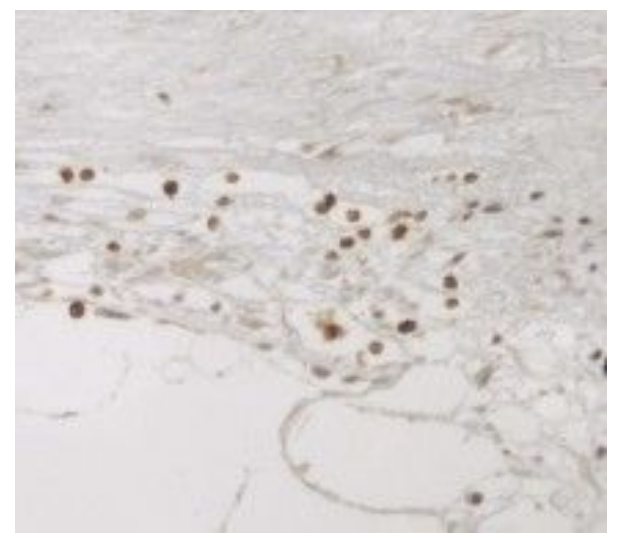

Abbildung 3.14: CD3-positive T-Zellen in der septierten Pseudointima in den Falten des Conduits; Vergrößerung 400fach; Präparat 4

\section{Graft-Gewebe}

Wie auch unter 3.1.1 beschrieben, bestand das eigentliche Graft-Gewebe vornehmlich aus deutlich gewellten elastischen Fasern sowie Kollagen-III- und weniger Kollagen-I-Fasern (Abb. 3.15A). Zwischen den Fasern zeigten sich spindelförmige Zellen, die positiv für SMA, Vimentin, meist auch SMMy und in einem Präparat zusätzlich positiv für h-Caldesmon und Desmin färbten (Abb. $3.15 \mathrm{~B})$.

Die Abgrenzung zwischen Pseudointima- und Graft-Gewebe war auf Grund der großen morphologischen Ähnlichkeit der beiden Schichten häufig schwierig. In der Mehrzahl der untersuchten Präparate fanden sich Kapillaren im GraftGewebe. Deren Wände färbten zum Teil positiv für SMA und SMMy, die innere Zellschicht war teilweise von-Willebrand- und Vimentin-positiv.

Wie in der Pseudointima waren auch im ursprünglichen Media-Gewebe der Homografts mehrfach Verkalkungsherde enthalten. In dem als Kalk-Spange bezeichneten Präparat 5 zeigte sich eine sehr ausgedehnte, schichtübergreifende Verkalkung. Auch die drei gestenteten Conduits wiesen Kalkareale im GraftGewebe auf, die sich zum Teil bis in die Pseudointima fortsetzten und ins Lumen vorwölbten. 

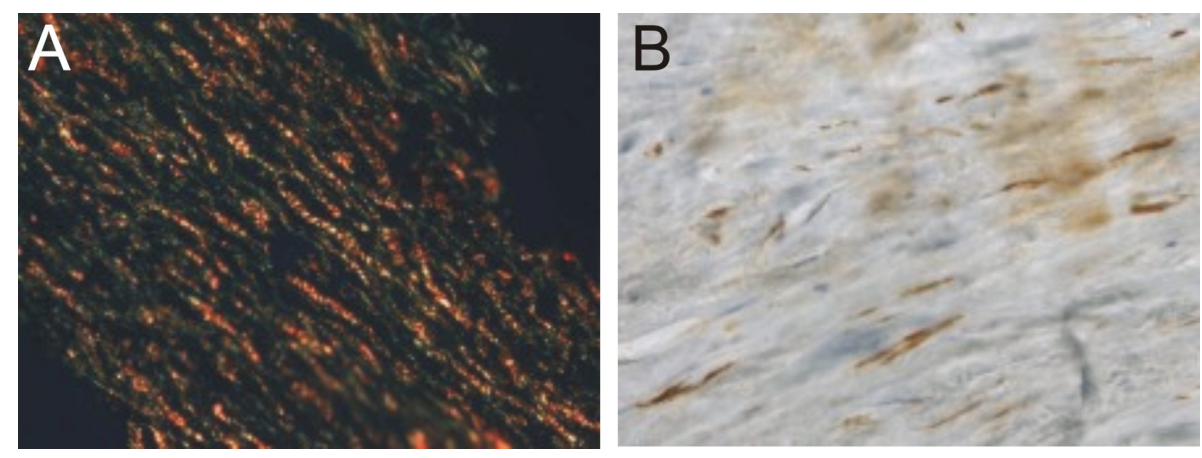

Abbildung 3.15: A Kollagenfasern der Subtypen I (orange) und III (grün) im HomograftGewebe; Pikrosirius-Rot-Färbung; Vergrößerung 200fach; Präparat 5; B SMA-positive fibromuskuläre Zellen im Homograft-Gewebe; Vergrößerung 400fach; Präparat 3

In mehreren Präparaten zeigte sich eine histomorphologische Zweiteilung des Homograft-Gewebes: Lumenseitig demarkierten sich die elastischen Fasern deutlich. In der Nähe der Adventitia ließ sich jedoch in der Färbung nach Richardson nur ein relativ homogen dunkel-blau bis -violett anfärbender Bereich erkennen, der vor allem aus Extrazellulärmatrix mit einer undeutlichen Faser-Anordnung und wenigen, schmalen Zellen bestand. Im stark verkalkten Präparat 5 zeigte sich ebenfalls ein Unterschied in der Morphologie des Grafts: Ein schmaler zur Pseudointima gewandter Abschnitt enthielt zwischen den Zellen wenig Extrazellulärmatrix. Deren Fasern waren verkalkt, ließen aber ihre gewellte Form noch erkennen. Der äußere Bereich des Graft-Gewebes jedoch wies mehr EZM-Einlagerungen auf, bei denen es sich zum Teil um KollagenIII-Fasern handelte. Dieser Abschnitt zeigte flächige Verkalkung, durch die in der von-Kossa-Färbung keine Gewebe-Strukturen mehr abgrenzbar waren. Im Gegensatz hierzu waren im Kalkareal der Pseudointima des Präparats 5 trotz intra- und extrazellulärer Verkalkung die Zellen und Fasern noch erkennbar. Das Graft-Gewebe in den Homografts mit Stent wies keine Zweiteilung auf. In der ursprünglichen Media waren im Gegensatz zu den anderen Schichten meist nur wenige inflammatorische Zellen nachweisbar. Im Homograft-Gewebe einiger Präparate zeigten sich Fäden der Anastomosen-Nähte. In deren Umgebung waren in der Färbung nach Richardson Entzündungszellen zu erkennen (Abb. 3.16).

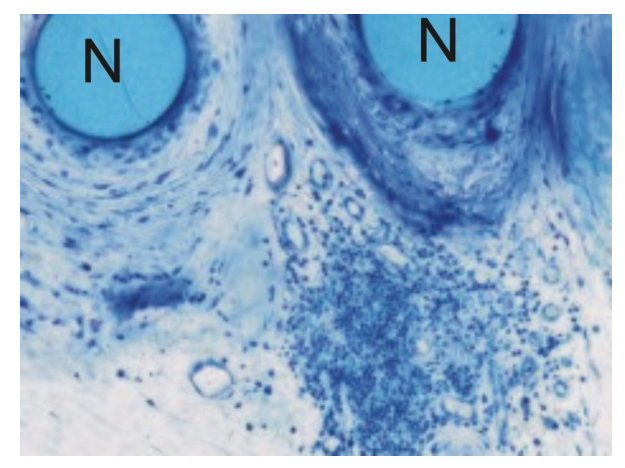

Abbildung 3.16: Entzündungszellen um Nahtmaterial (N) im Homograft-Gewebe; Färbung nach Richardson; Vergrößerung 100fach; Präparat 8 
In den meisten Präparaten ließen sich wenige, einzeln liegende CD68-positive Makrophagen zwischen den Graft-Fasern nachweisen. Im Graft-Gewebe der Präparate 3, 6 und 8 waren vergleichsweise viele CD68-positive Histiozyten enthalten. In Nummer 8 zeigten sich außerdem CD68-positive mehrkernige Riesenzellen. In der Mehrzahl der Präparate befanden sich CD3-positive TLymphozyten. Sie kamen einzeln und insgesamt in meist geringer Zahl vor. In Nummer 8 waren sie vor allem perivaskulär lokalisiert. Im Präparat 4 zeigten sich deutliche Infiltrate CD3-positiver T-Zellen am Übergang zur Adventitia. In einigen Präparaten konnte zusätzlich eine weitere Differenzierung in CD4positive T-Helferzellen und CD8-positive T-Killerzellen vorgenommen werden: In drei Präparaten färbten einzeln liegende Lymphozyten positiv für CD8 und in einem zeigten sich vereinzelte CD4-positive T-Zellen (Abb. 3.17A). Des Weiteren ließen sich im Graft-Gewebe dreier Präparate CD79-positive BLymphozyten anfärben. Sie kamen einzeln sowie zum Teil gruppiert liegend vor (Abb. 3.17B).
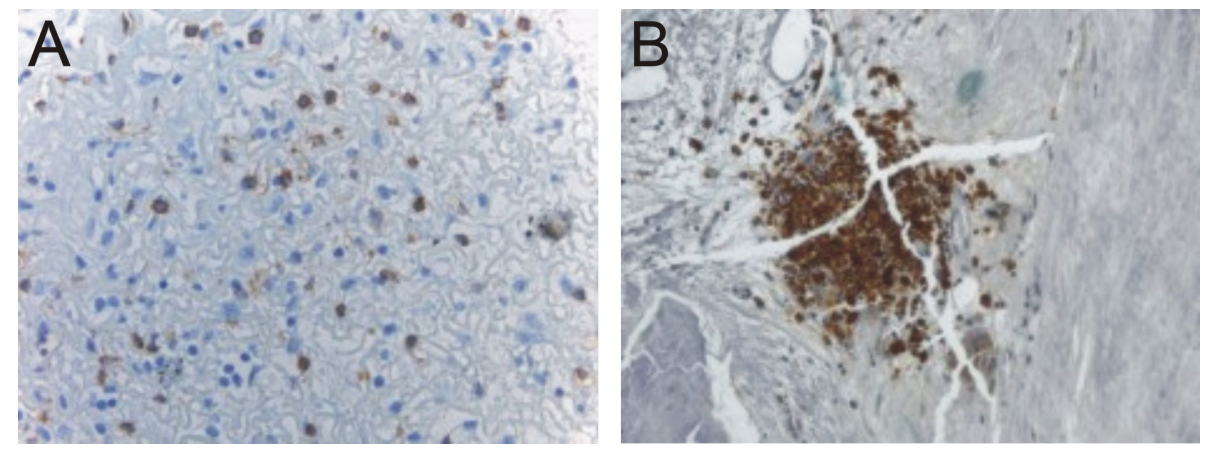

Abbildung 3.17: A CD8-positive T-Killerzellen zwischen den Fasern des Homograft-Gewebes; Vergrößerung 400fach; Präparat 4; B Infiltrat CD79-positiver B-Lymphozyten im GraftGewebe; Vergrößerung 200fach; Präparat 9

\section{Adventitia}

Die Adventitia der untersuchten Präparate zeigte das Bild einer typischen Gefäß-Adventitia. Es fand sich Bindegewebe mit gewellten Fasern. In der Pikrosirius-Rot-Färbung war vor allem Kollagen III und weniger ausgeprägt Kollagen I nachweisbar. Zwischen den Fasern lagen relativ wenige spindelförmige Zellen, die positiv für Vimentin und in zwei Präparaten zusätzlich stellenweise positiv für SMA färbten. Außerdem zeigten sich Fettzellen, MuskelfaserBündel sowie zahlreiche Kapillaren (Abb. 3.18). Die Zellen dieser Gefäßwände färbten in den meisten Präparaten positiv für SMA, seltener auch für SMMy, h-Caldesmon und Desmin (Abb. 3.19). Die innere Zellschicht der Kapillaren war von-Willebrand- und Vimentin-positiv. Teilweise befanden sich im Gewebe Ansammlungen von Erythrozyten. Mehrfach zeigten sich Fäden der Anastomosen-Nähte im Adventitia-Gewebe. Im Präparat 4 befanden sich in der Adventitia große Verkalkungsherde, die auch makroskopisch erkennbar waren. Dagegen beschränkte sich die Verkalkung in der Pseudointima dieses Präparats auf einzelne Zellen. In Nummer 10 setzte sich die ausgeprägte Verkalkung des Homograft-Gewebes auch in die Adventitia hinein fort. 

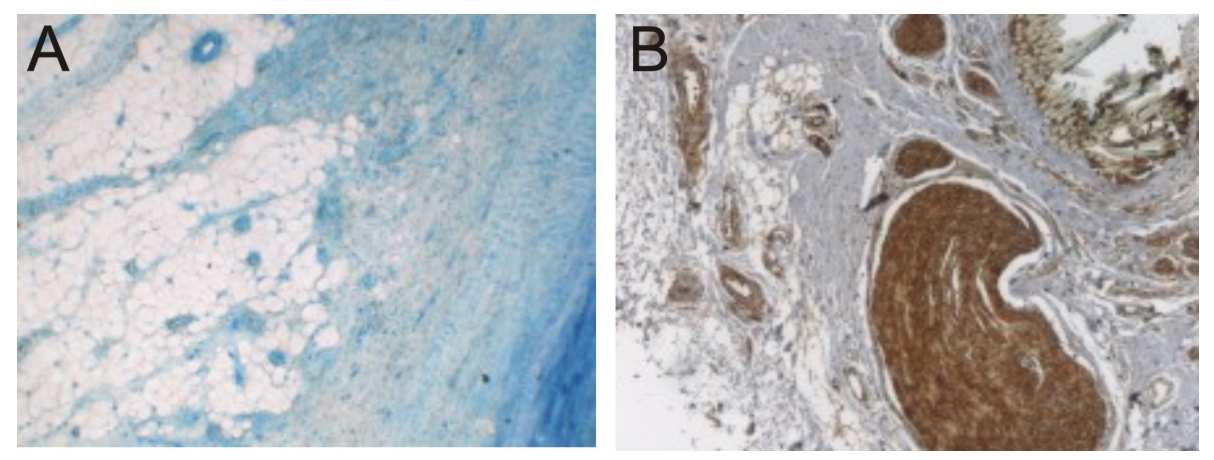

Abbildung 3.18: A Fettzellen in der Adventitia (nach rechts Übergang zum HomograftGewebe); Färbung nach Richardson; Vergrößerung 40fach; Präparat 3; B Vimentin-positive Zellen in Muskelfaser-Bündeln der Adventitia; Vergrößerung 100fach; Präparat 2

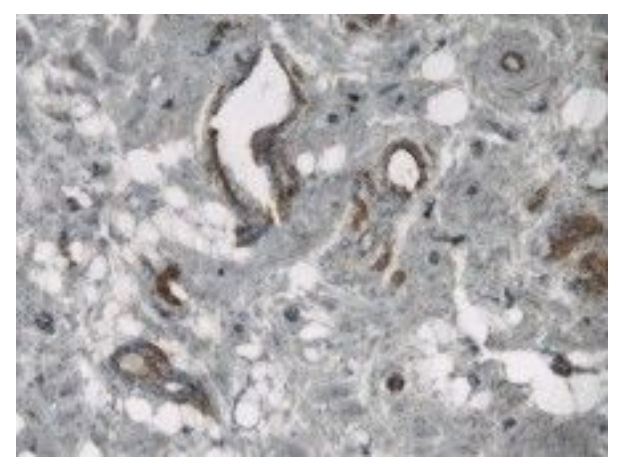

Abbildung 3.19: SMA-positive Zellen der Vasa vasorum in der Adventitia; Vergrößerung 100fach; Präparat 9

In allen untersuchten Präparaten waren in der Adventitia inflammatorische Zellen nachweisbar. Im Vergleich mit den anderen Schichten der Homografts war die Adventitia der am stärksten von Entzündung betroffene Bereich. So zeigten sich schon in der histomorphologischen Untersuchung in konventionellen Färbungen Lymphozyten und Granulozyten. In der Adventitia aller Präparate waren CD3-positive T-Lymphozyten nachweisbar. Sie kamen insgesamt meist in großer Zahl vor und fanden sich grundsätzlich einzeln liegend sowie häufig zusätzlich in Form von Lymphfollikeln (Abb. 3.20).

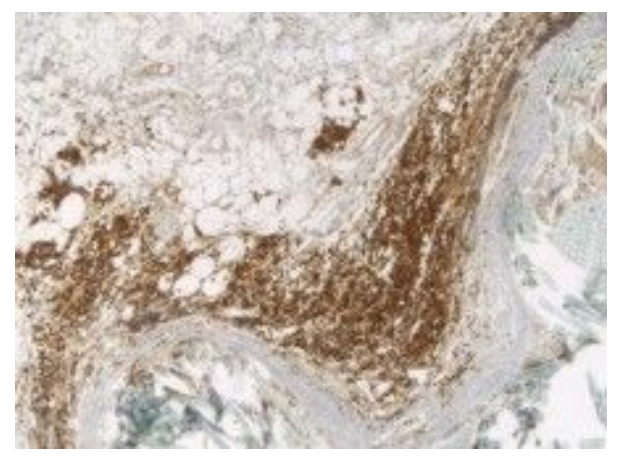

Abbildung 3.20: Ansammlung CD3-positiver T-Lymphozyten in der Adventitia; Vergrößerung 100fach; Präparat 2 
In den Präparaten 3, 7 und 8 waren die Infiltrate CD3-positiver Lymphozyten perivaskulär lokalisiert. In einigen Präparaten waren einzelne CD8-positive Lymphozyten in der Adventitia nachweisbar, in einem kamen vereinzelt CD4positive Zellen vor. Auch CD68-positive histiozytäre Zellen fanden sich in der Adventitia aller untersuchten Präparate. Häufig waren sie sehr zahlreich nachweisbar. Sie lagen generell einzeln. In mehreren Präparaten kamen zusätzlich CD68-positive mehrkernige Riesenzellen vor, die sich teilweise in unmittelbarer Nähe von Nahtmaterial befanden (Abb. 3.21). In der Adventitia der meisten Präparate konnten CD79-positive B-Lymphozyten nachgewiesen werden. Sie kamen mehrfach nur vereinzelt vor. Dagegen fanden sich im Präparat 4 neben einzeln liegenden B-Zellen auch drei Lymphozyten-Infiltrate, deren Zellen positiv für CD79 färbten (Abb. 3.22). Zwei Conduits ohne Nachweis CD79-positiver Zellen bei Verwendung des nicht-human-spezifischen Antikörpers wurden zusätzlich mit dem Antikörper gegen CD79alpha inkubiert. Dabei fanden sich in beiden Präparaten sowohl einzeln liegende als auch gruppierte CD79-positive B-Lymphozyten in der Adventitia. Mit dem Antikörper gegen CD138 konnten im Präparat 4 Plasmazellen als gereifte und ausdifferenzierte Vertreter der B-Zell-Reihe nachgewiesen werden. Sie fanden sich einzeln liegend sowohl verteilt in der Adventitia als auch im Übergangsbereich zum Graft-Gewebe (Abb. 3.23).

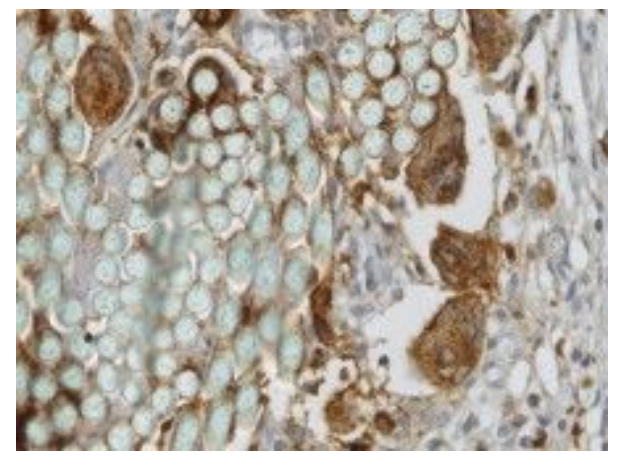

Abbildung 3.21: CD68-positive FKRZ um gewobene Fäden in der Adventitia; Vergrößerung 400fach; Präparat 2
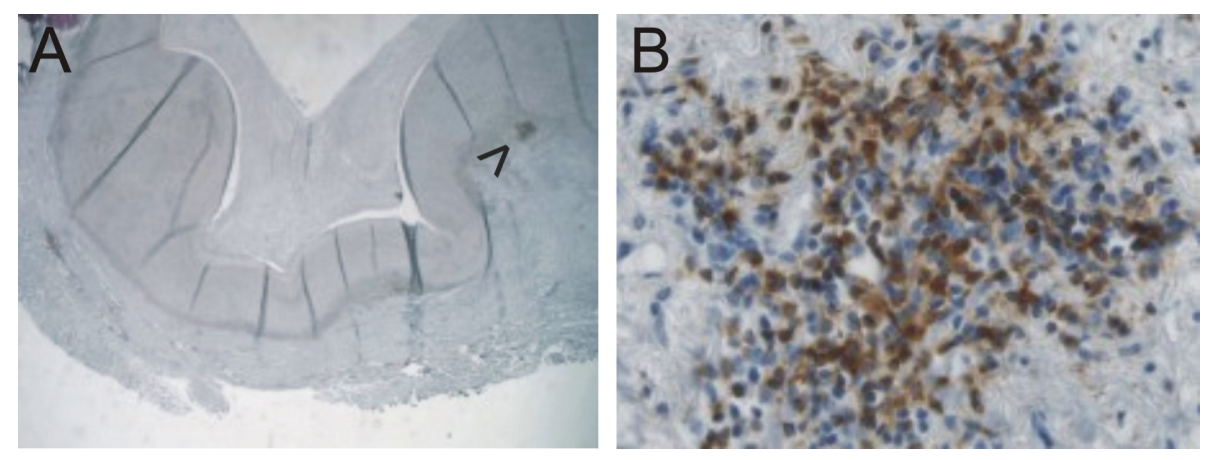

Abbildung 3.22: B-Zell-Infiltrat im Homograft-Gewebe an der Grenze zur Adventitia; CD79alpha; Vergrößerung 16fach bzw. 400fach; Präparat 4 


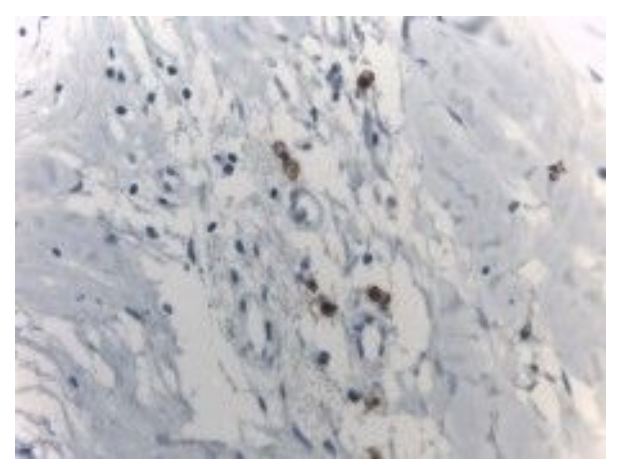

Abbildung 3.23: Plasmazellen im Homograft-Gewebe am Übergang zur Adventitia; CD138; Präparat 4

\section{Klappen}

In fünf Homografts konnten die Taschenklappen untersucht werden. Das Bindegewebe der Grafts setzte sich von der Conduit-Wand in die Klappen hinein fort (Abb. 3.24A). In der ungeordnet wirkenden Extrazellulärmatrix ließen sich elastische Fasern sowie wenige Zellen mit schmalem Zellkern erkennen (Abb. 3.24B). Immunhistochemisch waren keine Muskelzell-typischen Antigene nachweisbar.
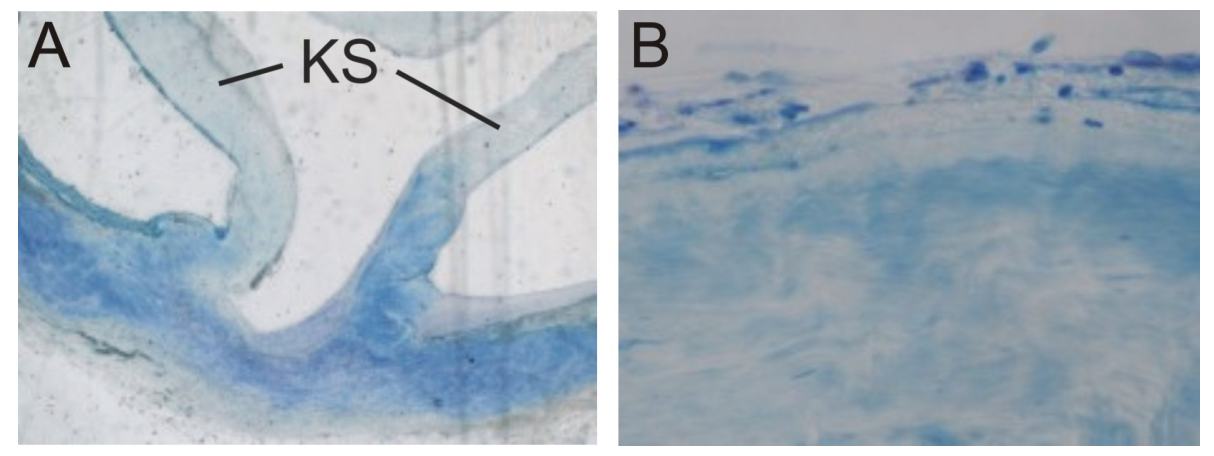

Abbildung 3.24: A Ansatz zweier Klappensegel (KS) an der Conduit-Wand; Färbung nach Richardson; Vergrößerung 16fach; Präparat 4; B zahlreiche Zellen im Pseudointima-Gewebe der Klappe, während das Graft-Gewebe der Klappe zellarm ist; Färbung nach Richardson; Vergrößerung 400fach; Präparat 1

Auch auf den Klappensegeln befand sich Pseudointima-Gewebe. Im Präparat 3 war dieses septiert und wies stellenweise viele inflammatorische Zellen auf (Abb. 3.25). Zudem fanden sich hier Kapillaren mit Erythrozyten sowie Einblutungen in Form großer extravasaler Erythrozyten-Ansammlungen, die dem makroskopischen Befund rötlich-brauner Flecken entsprachen (Abb. 3.26)

Im Präparat 4 befand sich in den Winkeln zwischen Conduit-Wand und Klappenabgängen, wie oben dargestellt, sehr ausgeprägt septiertes Material mit zahlreichen Entzündungszellen (Vgl. Abb. 3.5). In allen untersuchten Präparaten zeigte sich lumenseitig auf den Klappensegeln eine dünne Endothelschicht, die positiv für von-Willebrand-Faktor färbte. 

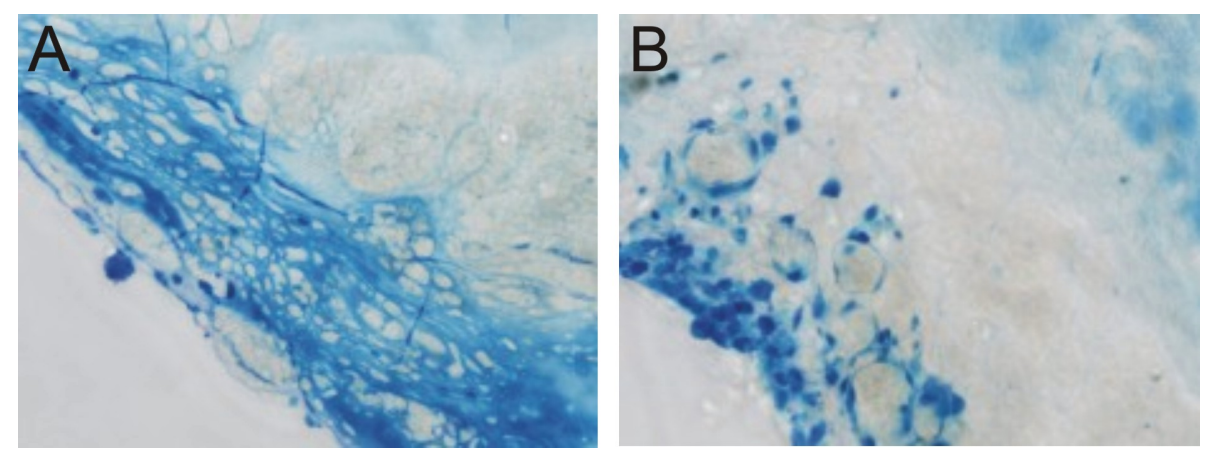

Abbildung 3.25: A septiertes Pseudointima-Gewebe an der Klappe; Färbung nach Richardson; Vergrößerung 400fach; Präparat 3; B Pseudointima-Gewebe an der Klappe mit Kapillaren und extravasalen Erythrozyten-Ansammlungen; Färbung nach Richardson; Vergrößerung 40fach; Präparat 3

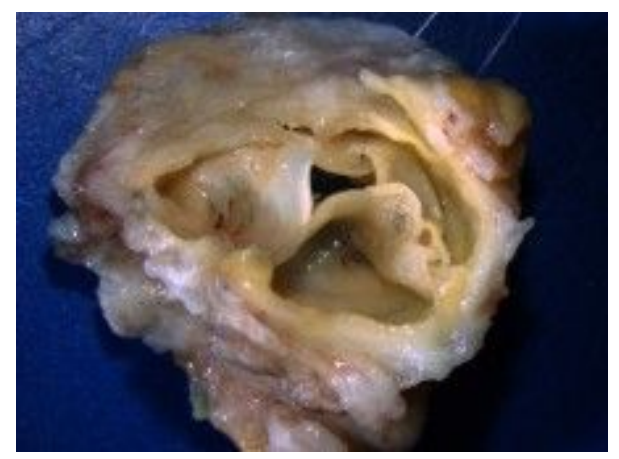

Abbildung 3.26: Einblutungen an den Klappensegeln; Präparat 3

Im Präparat 10 wies auch die Klappe Verkalkungsareale auf. Zudem zeigten sich in einem der Klappensinus thrombotisches Material mit vielen Erythrozyten und einigen Entzündungszellen sowie außerdem ein großer Kalkherd, der schon makroskopisch deutlich zu erkennen war (Abb. 3.27).
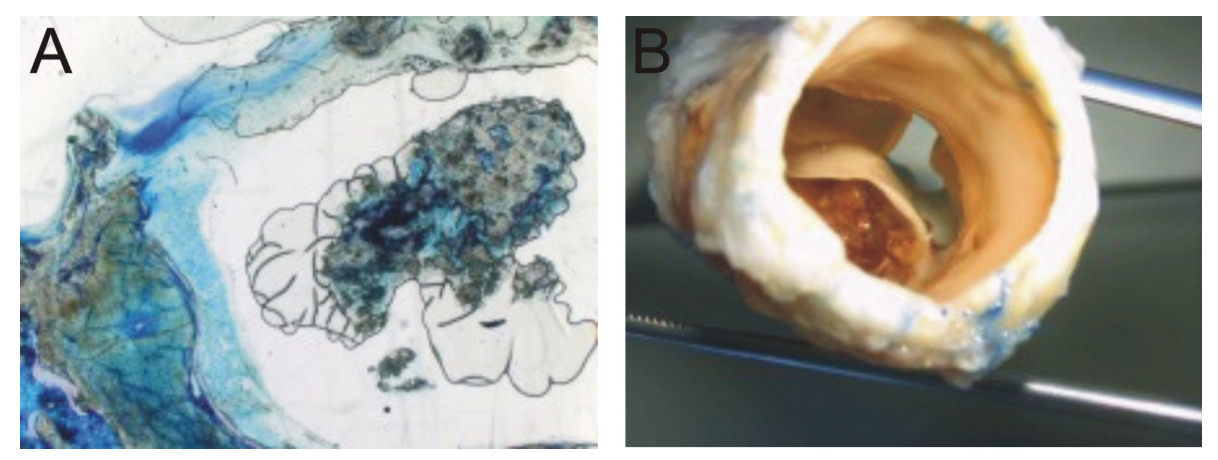

Abbildung 3.27: A verkalktes Klappensegel und Kalkherd im Klappensinus; Färbung nach Richardson; Vergrößerung 16fach; Präparat 10; B Präparat 10 nach Zuschnitt 
Unter den inflammatorischen Zellen in den Homograft-Klappen ließen sich einzeln liegende CD68-positive Makrophagen anfärben (Abb. 3.28). Auch vereinzelte CD3-positive T-Lymphozyten waren nachweisbar. Im septierten Material an den Klappenursprüngen des Präparats 4 ließen sich nach histomorphologischen Kriterien Lymphozyten und Granulozyten identifizieren.
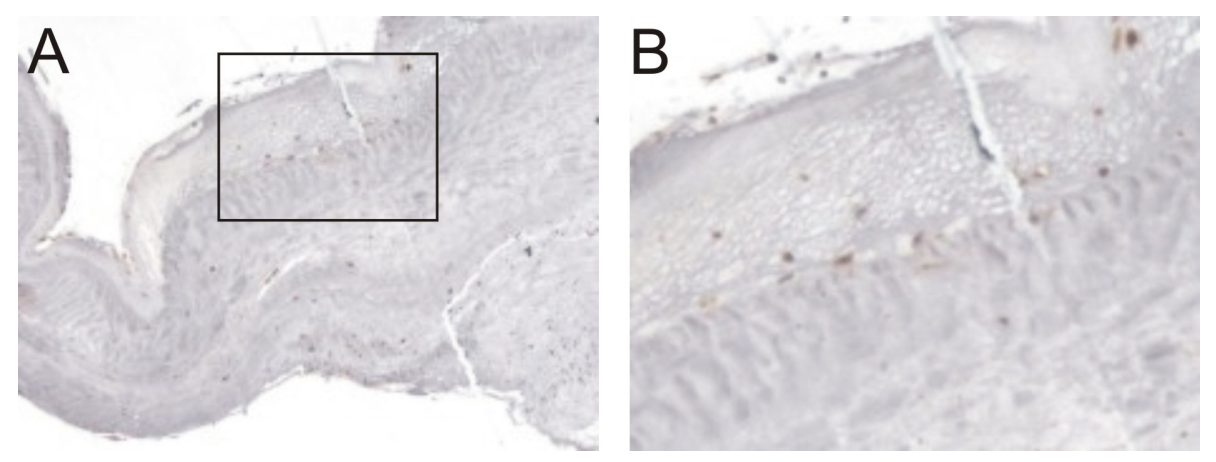

Abbildung 3.28: CD68-positive Histiozyten in der Klappe; Vergrößerung 40fach bzw. ca 200fach; Präparat 3

\section{Stents}

Die Metallstreben der Stents in den drei betreffenden Präparaten zeigten eine glatte Oberfläche ohne Anhalt für korrosionsbedingte Veränderungen. Zum Teil ragten sie frei in das Conduit-Lumen vor, meist aber waren sie von Gewebe der Pseudointima umgeben. Diese wies in der makroskopischen Untersuchung eine weißlich-glatte Oberfläche auf (Abb. 3.29). Die feingewebliche Analyse zeigte, dass sie in Stent-Nähe, ebenso wie in den anderen Bereichen auch, vornehmlich aus Fasern und fibromuskulären Zellen bestand.

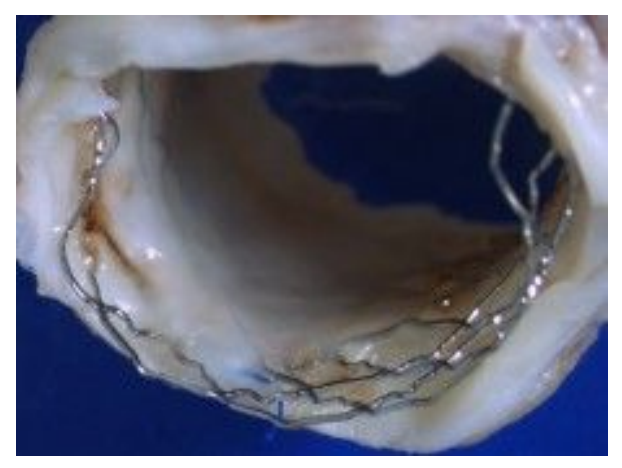

Abbildung 3.29: makroskopisch unauffällige Oberfläche der Pseudointima mit überwachsenen Stentstreben; Präparat 8

In das Homograft 9 waren zwei Stents implantiert worden. Die Implantationszeit des ersten betrug fünf Jahre. Bei dem zweiten handelte es sich um ein Stent-Graft mit PTFE-Membran, das für zwei Jahre und zwei Monate implantiert gewesen war. In den untersuchten Präparaten war der zuletzt implantierte Stent zu erkennen. Es zeigte sich ausgedehntes Pseudointima-Gewebe sowohl zwischen Homograft und der PTFE-Membran als auch lumenseitig des Stent- 
Grafts (Abb. 3.30A). Beide Pseudointima-Lagen waren faserreich und enthielten spindelförmige Zellen, die positiv für SMA, SMMy und Vimentin färbten (Abb. 3.30B).
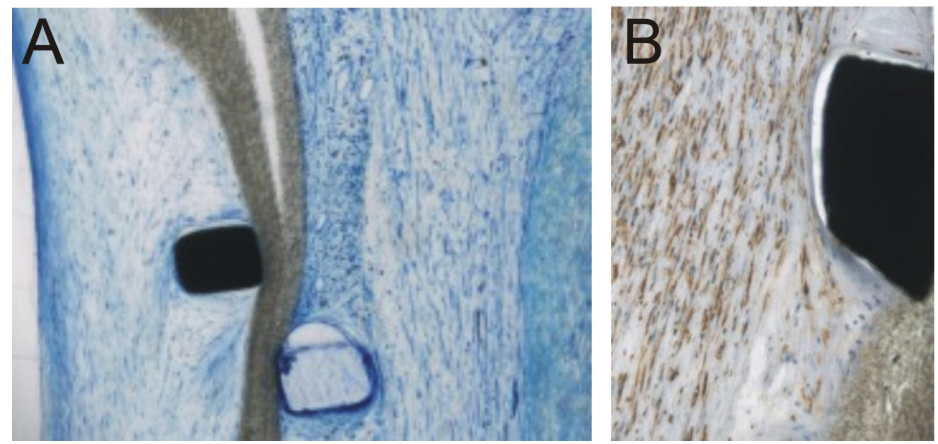

Abbildung 3.30: A unterschiedliche Bereiche der Pseudointima (links im Bild Lumen); Färbung nach Richardson; Vergrößerung 100fach; Präparat 9; B SMA-positive Zellen im lumenseitig (links im Bild) gelegenen Bereich der Pseudointima; Vergrößerung 200fach; Präparat 9

Im äußeren, dem Homograft zugewandten Teil der Pseudointima fanden sich in der Nähe des Stents viele Lakunen, einige Kapillaren und stellenweise Erythrozyten. Außerdem ließen sich in diesem Bereich in der Färbung nach Richardson nach histomorphologischen Kriterien deutlich mehr Entzündungszellen als in der lumenseitigen Pseudointima-Schicht erkennen. Mit den Antikörpern gegen CD3 und CD79 waren auf beiden Seiten der PTFE-Membran T-Lymphozyten und sehr wenige B-Lymphozyten nachweisbar, die jeweils einzeln in der Pseudointima lagen. Außerdem ließen sich CD68-positive Makrophagen anfärben: In der Pseudointima-Lage zwischen PTFE-Membran und Homograft zeigten sich zahlreiche Histiozyten, in der lumenseitigen Pseudointima-Schicht nur wenige, kleinere Histiozyten. In der Nähe der Stent-Streben befanden sich zu beiden Seiten der PTFE-Membran einige CD68-positive mehrkernige Riesenzellen (Abb. 3.31).

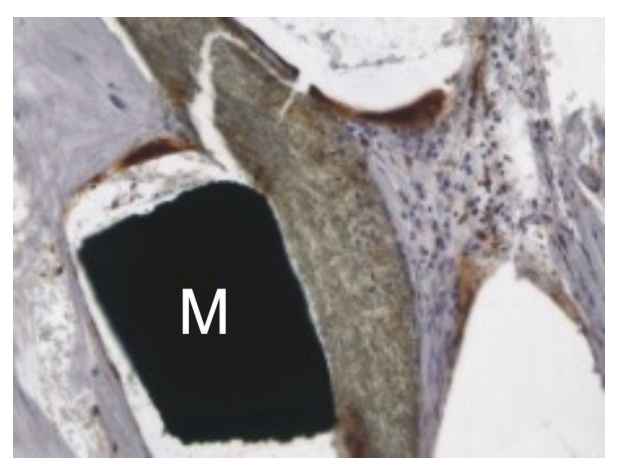

Abbildung 3.31: Histiozyten und Fremdkörperriesenzellen; Metallstreben (M) des Stents teilweise herausgelöst (nach links Lumen); CD68; Vergrößerung 200fach; Präparat 9

Die Implantationszeit des Stents im Präparat 8 betrug zwei Jahre und fünf Monate. Insgesamt war die Pseudointima des Präparates vergleichsweise kräftig ausgeprägt. Sie bestand aus Kollagenfasern, wobei in der Pikrosirius- 
Rot-Färbung fast ausschließlich der Subtyp I nachweisbar war (Abb. 3.32A). Zwischen den Fasern lagen lange Zellen, die positiv für SMA, SMMy, Vimentin, h-Caldesmon und Desmin färbten (Abb. 3.32B).
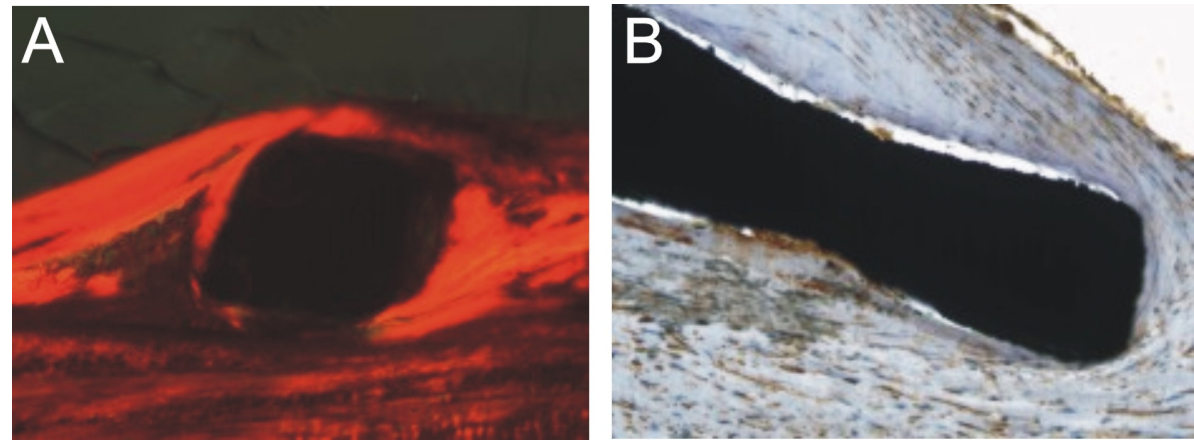

Abbildung 3.32: A Kollagen I (orange) in der Pseudointima um die Stent-Streben; Pikrosirius-Rot-Färbung; Vergrößerung 200fach; Präparat 8; B Vimentin-positive fibromuskuläre Zellen in der Pseudointima um den Stent; Vergrößerung 200fach; Präparat 8

Den Stent-Streben benachbart zeigte sich die Pseudointima in der Färbung nach Richardson stellenweise amorph mit nur undeutlicher Gewebe-Struktur. Die Kalkeinlagerungen der Pseudointima reichten zum Teil bis in die Nähe des Stent-Materials. Den Streben benachbart fanden sich vereinzelte CD3-positive T-Lymphozyten.

Der Stent im Homograft Nummer 7 war für zwei Jahre und zehn Monate implantiert gewesen. Auch lumenseits seiner Streben befand sich zum Teil Gewebe der Pseudointima. Deren Verkalkungen setzten sich stellenweise bis zum Metall fort, einige Stent-Streben waren zirkulär von Kalk umgeben (Abb. 3.33).

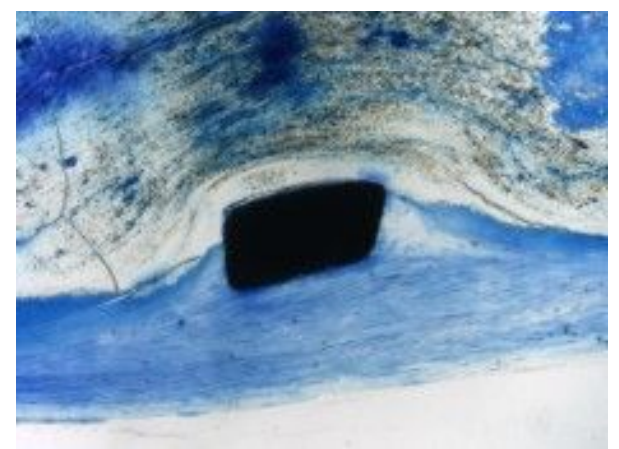

Abbildung 3.33: Verkalkungsarelae in der Pseudointima (unten Lumen); Färbung nach Richardson; Vergrößerung 100fach; Präparat 7 


\subsection{Rindervenengrafts}

Die Implantationszeiten der untersuchten bovinen Xenografts lagen zwischen einem Jahr und acht Monaten und fünfeinhalb Jahren.

\subsubsection{Histopathologische Auswertung der Rindervenengrafts}

\section{Pseudointima und Endothel}

Die Gefäßwände der drei untersuchten Conduits wiesen in der makroskopischen Untersuchung lumenseitig eine weißlich-glatte Oberfläche auf (Abb. 3.34A).
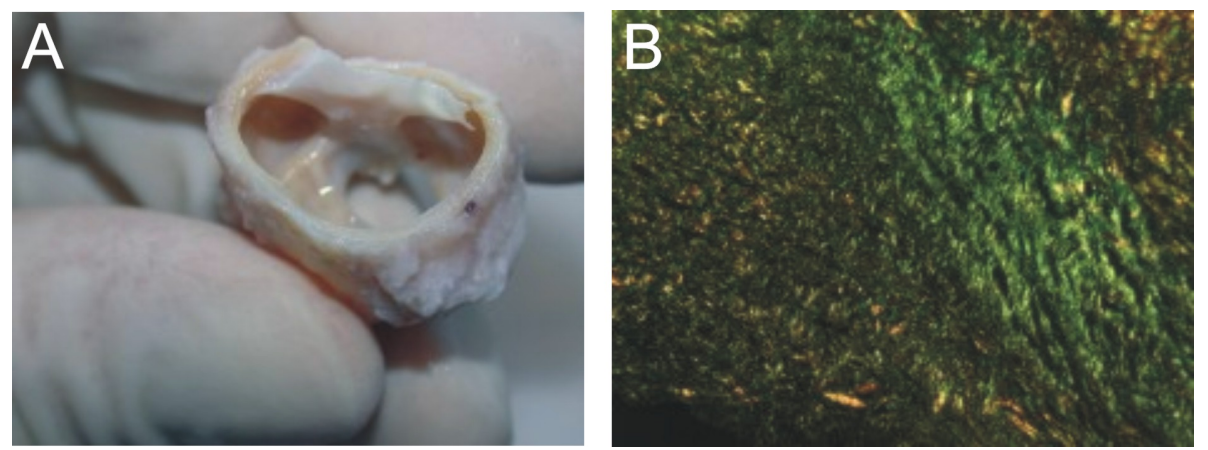

Abbildung 3.34: A Präparat 13 nach Zuschnitt; B Kollagen-III-Fasern in der Pseudointima (unten links Lumen); Pikrosirius-Rot-Färbung; Vergrößerung 200fach; Präparat 13

Auch mikroskopisch fand sich als innere Schicht eine unterschiedlich ausgeprägte bindegewebige Pseudointima. Sie setzte sich zusammen aus gewellten Fasern mit eingelagerten spindelförmigen Zellen. Teilweise enthielt sie zudem Entzündungszellen.

Die Pseudointima des Präparats 13 bestand aus elastischen und feinen, diffusen Kollagenfasern, wobei in der Pikrosirius-Rot-Färbung der Fasertyp III gegenüber Typ I überwog (Abb. 3.34B). Lumenseitig ließen sich wenige Fibrinfäden anfärben.

Zwischen den Fasern befanden sich lange, dünne Zellen mit ovalem bis länglich-ovalem Zellkern. Sie zeigten eine positive Färbung für SMA, SMMy, Vimentin, h-Caldesmon und Desmin. Abschnittsweise waren zahlreiche Kapillaren enthalten. Deren lumenseitige Zellschicht färbte positiv für vonWillebrand-Faktor. Die Zellen der Kapillarwand waren SMA- und Vimentinpositiv. In der Pseudointima waren besonders perivaskulär kleine Gruppen von Entzündungszellen nachweisbar. Diese färbten positiv für die Antikörper gegen CD3, CD79alpha und CD138 (Abb. 3.35).

CD3-, CD4- und CD8-positive T-Lymphozyten zeigten sich zudem auch einzeln und insgesamt in vergleichsweise geringer Zahl. Darüber hinaus befanden sich wenige CD68-positive Makrophagen in der Pseudointima. Auch die schmale Pseudointima im Präparat 12 bestand aus feinen elastischen und Kollagen-III-Fasern, die dicht aneinander gelagert waren. Die unterschiedlich zahlreich enthaltenen spindelförmigen Zellen färbten positiv für SMA, Vimentin und zum Teil auch für h-Caldesmon. Stellenweise waren histomorphologisch 


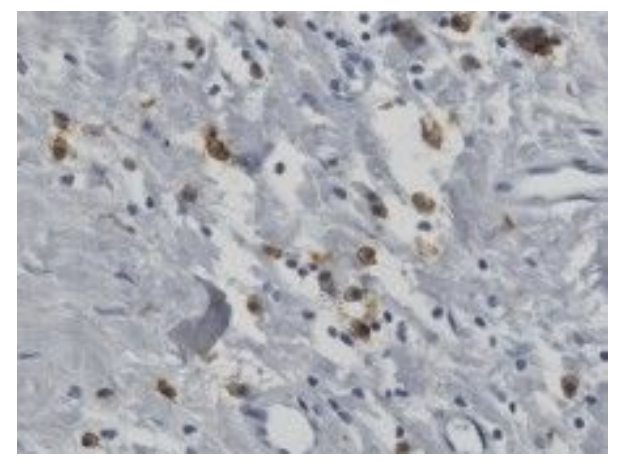

Abbildung 3.35: CD138-positive Plasmazellen perivaskulär in der Pseudointima; Vergrößerung 400fach; Präparat 13

einige Entzündungszellen zu erkennen. Mit den verwandten Antikörpern gegen CD3, CD68, CD79, CD79alpha und CD138 war jedoch keine spezifische Anfärbung zu erkennen.

Im Conduit Nummer 11 zeigte sich die Pseudointima unterschiedlich stark ausgeprägt und wölbte sich stellenweise in das Lumen hinein vor. Sie bestand aus elastischen Fasern sowie Kollagen-I- und Kollagen-III-Fasern in überwiegend ausgeglichenem Verhältnis. Es zeigte sich deutlich eine positive Färbung für Fibrin. Außerdem kamen wenige längliche SMA-positive Zellen und wenige Entzündungszellen vor. Unter den inflammatorischen Zellen färbten einige positiv für die Antikörper gegen CD3, CD4 und CD8 sowie CD68. Lumenseitig befand sich an der bindegewebig organisierten Pseudointima-Schicht stellenweise zusätzlich eine schmale Lage thrombotischer Pseudointima. Diese bestand aus amorphem Material und Fibrinsepten, zwischen denen sich zum Teil längliche Zellen fanden. In einem Abschnitt thrombotischer Pseudointima zwischen den Klappenabgängen färbten spindelförmige Zellen positiv für h-Caldesmon und Desmin. In der Pikrosirius-Rot-Färbung zeigten sich auch hier Kollagen-III-Fasern. Außerdem fanden sich wiederum Entzündungszellen: Es waren CD3-positive T-Lymphozyten nachweisbar, die überwiegend einzeln vorkamen (Abb. 3.36).

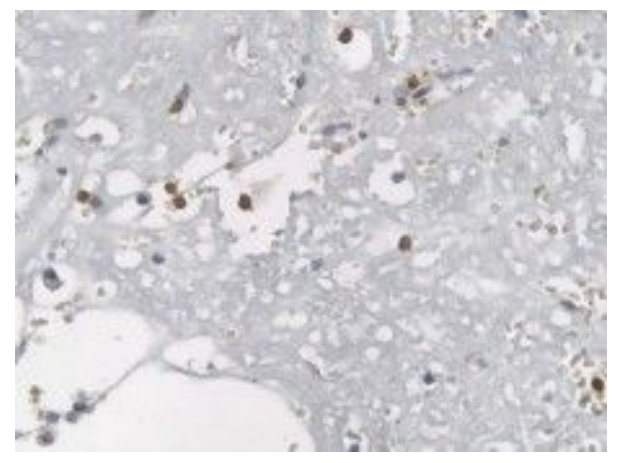

Abbildung 3.36: CD3-positive T-Lymphozyten in der Pseudointima zwischen den Klappenabgängen; Vergrößerung 400fach; Präparat 11 
Der Antikörper gegen CD79alpha färbte gruppierte B-Lymphozyten. An der gleichen Stelle waren auch mehrere CD138-positive Plasmazellen zu finden. In der thrombotischen Pseudointima waren kleine Kapillaren zu erkennen. Deren lumenseitige Zellschicht war von-Willebrand-Faktor-positiv. Die Zellen der Kapillarwände färbten positiv für SMA, SMMy, Vimentin, h-Caldesmon und Desmin. Die Pseudointima des Präparats 11 enthielt ausgeprägte Verkalkungen, die auch makroskopisch erkennbar waren. Bei diesen handelte es sich teilweise um größere, flächige Einschlüsse. Es zeigten sich in der von-Kossa-Färbung aber auch fein verteilte verkalkte Faserstrukturen. Außerdem fand sich im Conduit Nummer 11 als einzigem Präparat ein großfächiger wandständiger Thrombus (Abb. 3.37).
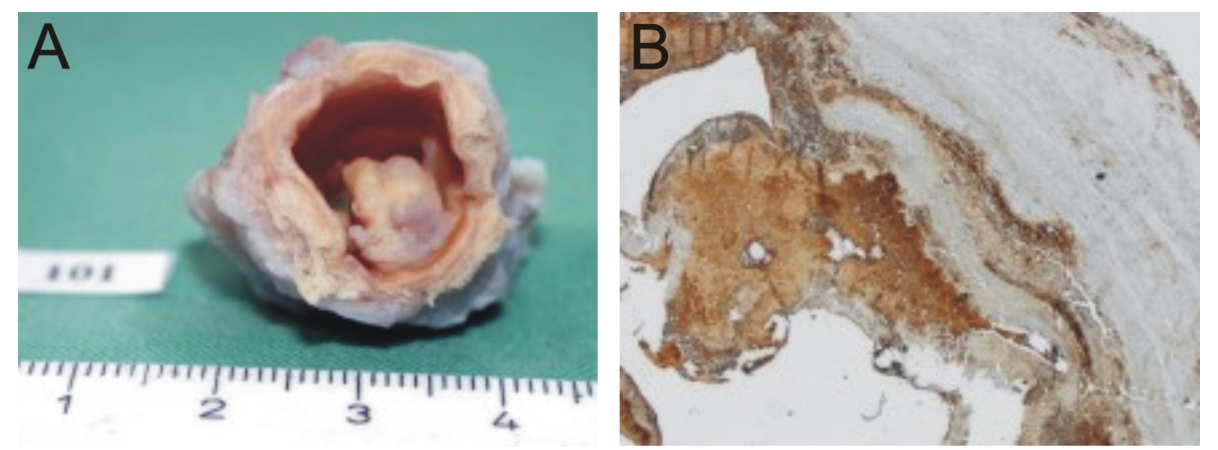

Abbildung 3.37: A makroskopisch erkennbarer wandständiger Thrombus im Präparat 11 nach Zuschnitt; Präparat 11; B wandständiger Thrombus; Fibrinfärbung; Vergrößerung 16 fach

Dieser bestand im Inneren aus amorphem Material mit aufgelockerter Struktur sowie Erythrozyten und wenigen Entzündungszellen. Das amorphe Material färbte zu großen Teilen positiv für Fibrin (Abb. 3.37B). In der von-KossaFärbung zeigten sich flächige Kalkeinschlüsse im Zentrum des Thrombus (Abb. 3.38). Es waren keine Kollagenfasern enthalten.

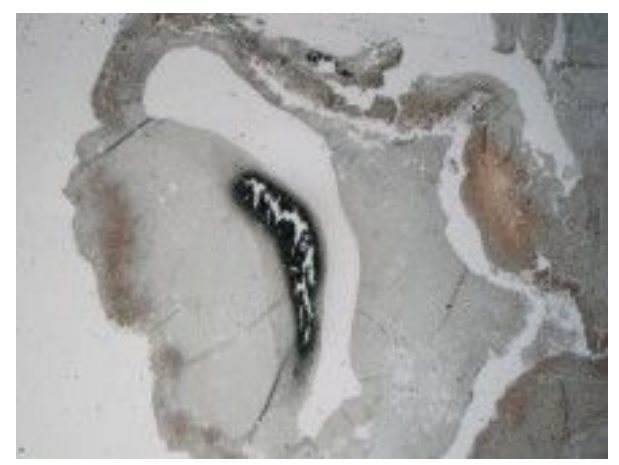

Abbildung 3.38: Kalkeinschlüsse im Thrombus; Färbung nach von Kossa; Vergrößerung 16fach; Präparat 11

Der parietale Thrombus wies lumenseitig eine schmale Lage bindegewebig organisierten Pseudointima-Gewebes auf (Abb. 3.39). Unter den Entzündungszellen im thrombotischen Material ließen sich immunhistochemisch vor allem einzelne CD68-positive Histiozyten und auch Fremdkörperriesenzellen identi- 

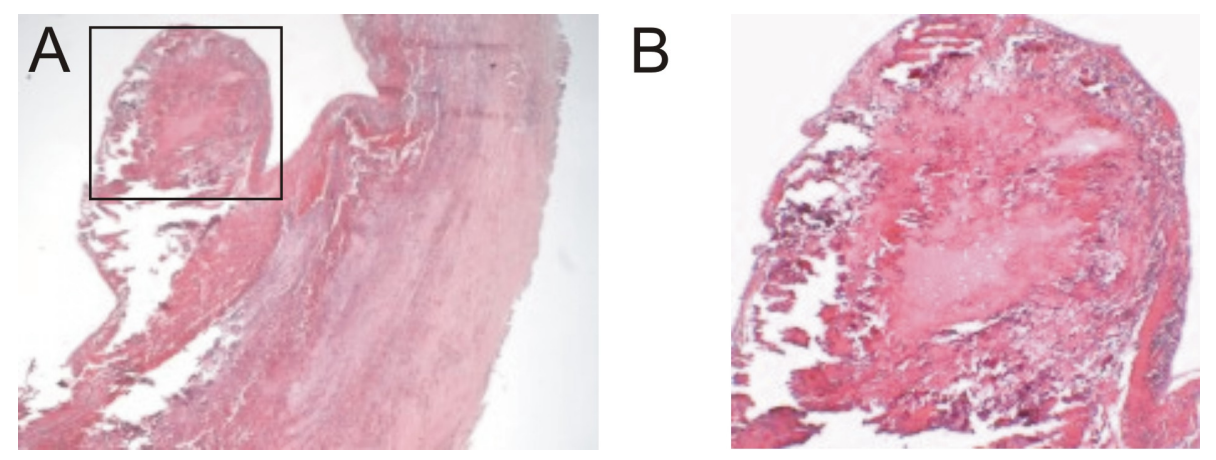

Abbildung 3.39: wandständiger Thrombus mit organisierten und amorphen Bereichen sowie Entzündungszellen; HE; Vergrößerung 16fach bzw. 40fach; Präparat 11

fizieren (Abb. 3.40A). In den Präparaten 13 und 11 zeigte sich lumenseitig sowohl an der Conduit-Wand als auch - im Präparat 11 - an der Klappe und dem parietalen Thrombus eine dünne von-Willebrand-Faktor-positive Endothelzelllage (Abb. 3.40B).
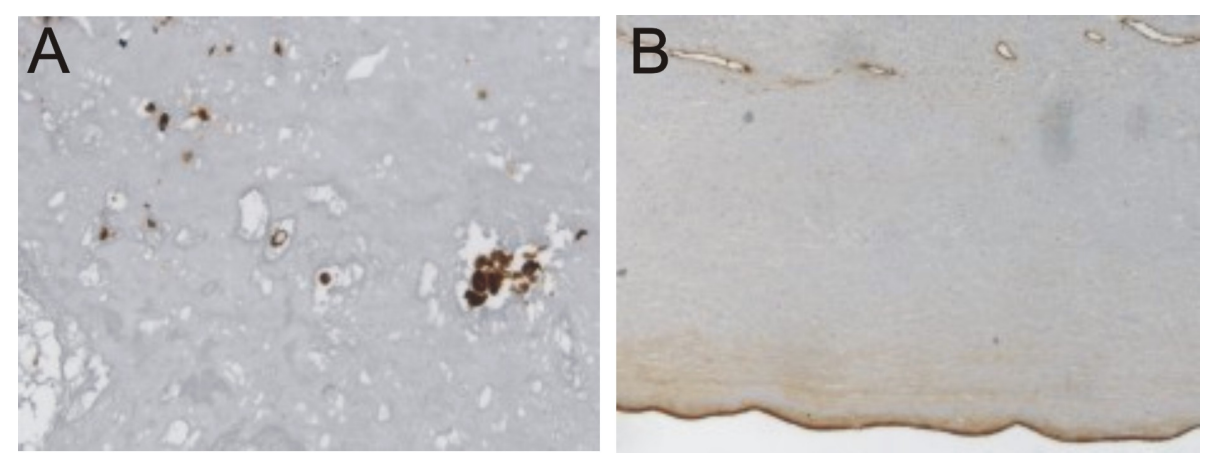

Abbildung 3.40: A Makrophagen in der amorphen Substanz des Thrombus; CD68; Vergrößerung 200fach; Präparat 11; B Endothel lumenseitig an der Pseudointima und in den Kapillaren (unten Lumen); von-Willebrand-Färbung; Vergrößerung 100fach; Präparat 13

\section{Graft-Gewebe}

Das avitalisierte Rindervenen-Gewebe war in allen drei vorliegenden Conduits erhalten. Histologisch zeigte sich besonders in den Präparaten 11 und 13 eine Zweiteilung des fibrösen Gewebes in einen Abschnitt mit stark gewellten Fasern und einen mit wesentlich weniger stark gewellten Fasern (Abb. 3.41). Der Pseudointima benachbart fanden sich deutlich gewellte Fasern, bei denen es sich unter anderem um grobe elastische Fasern handelte. Zwischen ihnen waren spindelförmige Zellen erkennbar. Im Präparat 11 ließen sich in den Bindegewebs-Färbungen relativ wenige Kollagenfasern nachweisen, wobei die Subtypen I und III gleichmäßig vorkamen. Die Fasern waren von stellenweise viel amorpher Substanz umgeben. Die spindelförmigen Zellen färbten positiv für SMA. Zudem waren zahlreiche inflammatorische Zellen und einige Kapillaren enthalten. 


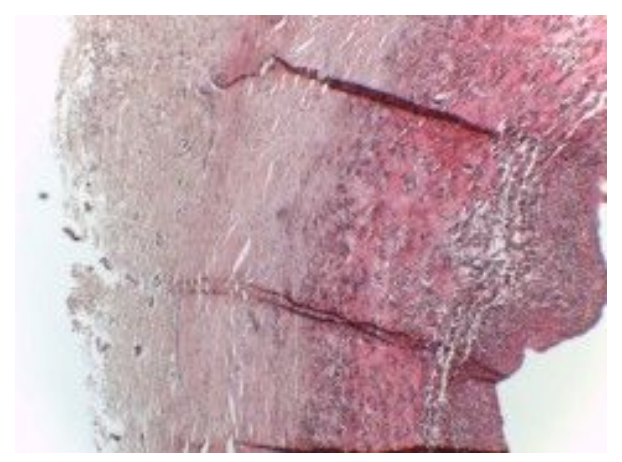

Abbildung 3.41: Schichtung der Wand, im Bild rechts (unten rechts Lumen) dichte gewellte Fasern der Pseudointima, angrenzend bindegewebiges Graft-Gewebe; HE; Vergrößerung 40fach; Präparat 11

In der von-Kossa-Färbung zeigten sich verkalkte faserige Strukturen im Graft-Gewebe an der Basis des wandständigen Thrombus. Im übrigen lumenseitigen Bereich des Grafts waren diffuse Kalkeinschlüsse erkennbar.

Im Präparat 13 fanden sich im gewellten Graft-Abschnitt stellenweise KollagenIII-Fasern. Die spindelförmigen Zellen färbten positiv für Vimentin und SMA (Abb. 3.42). Außerdem waren einige Entzündungszellen sowie Kapillaren enthalten. Im äußeren Graft-Bereich mit kaum gewellten Fasern ließen sich in den Bindegewebs-Färbungen ausschließlich Fasern des Kollagen-Subtyps III anfärben, während Kollagen I nicht nachweisbar war. Zwischen den Fasern befanden sich hier gleichmäßig einzeln liegende, lange Vimentin-positive Zellen. Es waren keine Kapillaren erkennbar.
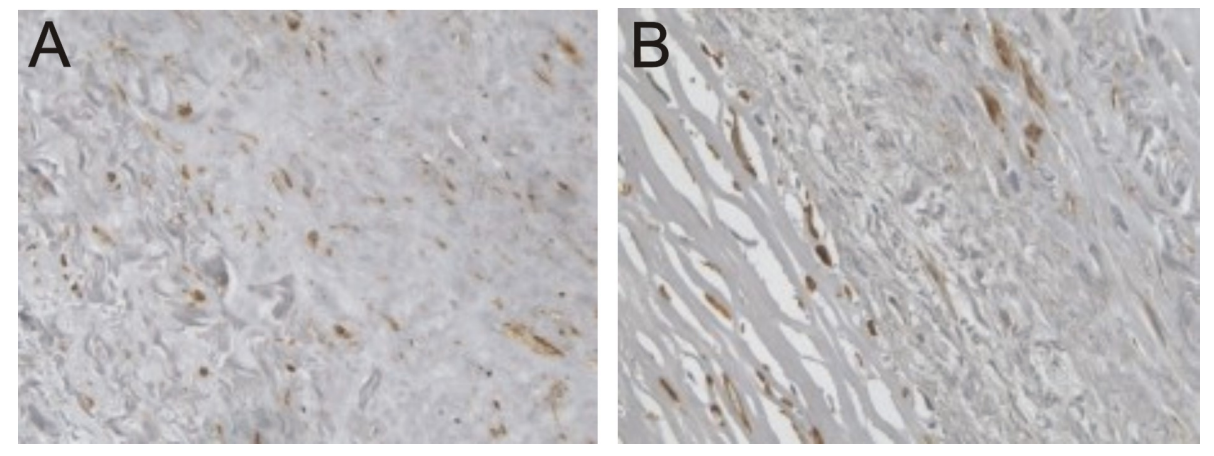

Abbildung 3.42: A Vimentin-positive Zellen im Graft-Gewebe; rechts im Bild gerade Fasern, links gewellte Fasern; Vergrößerung 200fach; Präparat 13; B SMA-positive fibromuskuläre Zellen und Fasern unterschiedlicher Struktur im Graft-Gewebe (links im Bild zum Lumen weisender Anteil des Gewebes); Vergrößerung 400fach; Präparat 13

Auch im Conduit 12 unterschieden sich die Fasern des Grafts. Hier fand sich jedoch lumenseitig eine Schicht parallel verlaufender, kaum gewellter Fasern. Peripher lagen die Fasern hingegen dichter, sie waren stärker gewellt und weniger parallel angeordnet. In den Bindegewebs-Färbungen zeigten sich feine gewellte Fasern des Kollagen-Typs III, aber keine elastischen und keine Kollagen-I-Fasern. Zwischen den Fasern befanden sich Zellen, die positiv für SMA, Vimentin und h-Caldesmon färbten. Es waren sehr wenige inflam- 
matorische Zellen und einige Kapillaren zu erkennen. Insgesamt enthielt das Graft-Gewebe des Präparats 13 im Vergleich mit den anderen Schichten die wenigsten Entzündungszellen. Es kamen wenige CD3-positive T-Zellen und CD68-positive Makrophagen im gewellten Graft-Abschnitt vor, im Teil mit kaum gewellten Fasern zeigten sich besonders wenige Entzündungszellen.

Im Präparat 11 waren im zum Lumen gewandten Abschnitt des Grafts mit den stark gewellten Fasern im Vergleich die meisten inflammatorischen Zellen zu finden. CD3-positive T-Lymphozyten lagen einzeln und an der Klappenbasis als kleines Infiltrat. Einzelne Lymphozyten färbten positiv für CD4 und CD8. Außerdem waren viele CD68-positive Makrophagen enthalten (Abb. 3.43A).
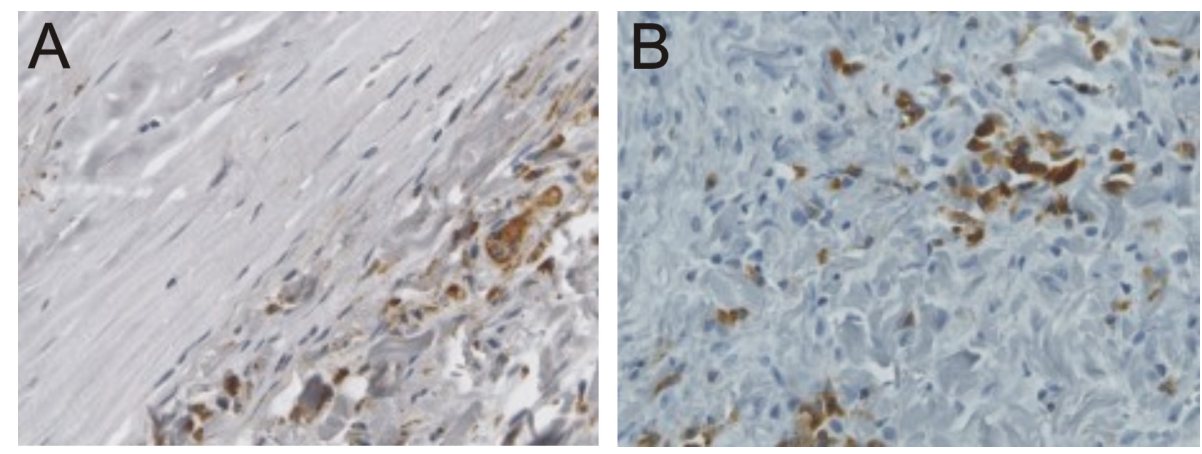

Abbildung 3.43: A Fremdkörperriesenzellen und Histiozyten zwischen den stark gewellten Fasern des Grafts; nur wenige, kleine Histiozyten in der Schicht mit weniger stark gewellten Fasern; CD68; Vergrößerung 400fach; Präparat 11; B gruppierte B-Lymphozyten im gewellten Graft-Bereich in Klappen-Nähe; CD79alpha; Vergrößerung 400fach; Präparat 11

Der Antikörper gegen CD79alpha färbte im gewellten Graft-Teil deutlich einzeln liegende B-Lymphozyten sowie eine Gruppe von B-Zellen an der Klappenbasis an (Abb. 3.43B). An der gleichen Stelle zeigten sich auch gruppiert CD138-positive Plasmazellen, die ansonsten in sehr geringer Zahl einzeln vorkamen. Im äußeren Bereich des Grafts mit kaum gewellten Fasern ließen sich dagegen insgesamt nur wenige Entzündungszellen nachweisen. Die CD79positiven B-Lymphozyten waren kleiner als in den anderen Bereichen.

\section{Adventitia}

In allen drei Präparaten war das Graft-Gewebe außen von einer Adventitia umgeben. Diese war reich an gewellten Fasern, zu denen immer auch feine elastische Fasern zählten, und enthielt zudem spindelförmige Zellen, inflammatorische Zellen, viele Kapillaren mit umgebenden feinen elastischen Fasern sowie unterschiedlich ausgeprägt Fettzellen und Muskelfasern (Abb. 3.44, 3.45).

Der Conduit Nummer 11 zeigte sich bei der makroskopischen Untersuchung außen von wenig unauffälligem Muskel- und Bindegewebe umgeben. Auch mikroskopisch fanden sich in der Adventitia des Präparats dünne Muskelbündel, deren Zellen positiv für h-Caldesmon und Desmin färbten. Neben elastischen Fasern waren in der Adventitia lange Kollagen-I- und -III-Fasern nachweisbar. Die beiden Subtypen waren größtenteils in ausgeglichenem Verhältnis vorhanden. Histomorphologisch zeigten sich Lymphozyten und wenige Granulozyten. Die Färbungen mit Antikörpern gegen CD3-, CD4-und CD8- so- 

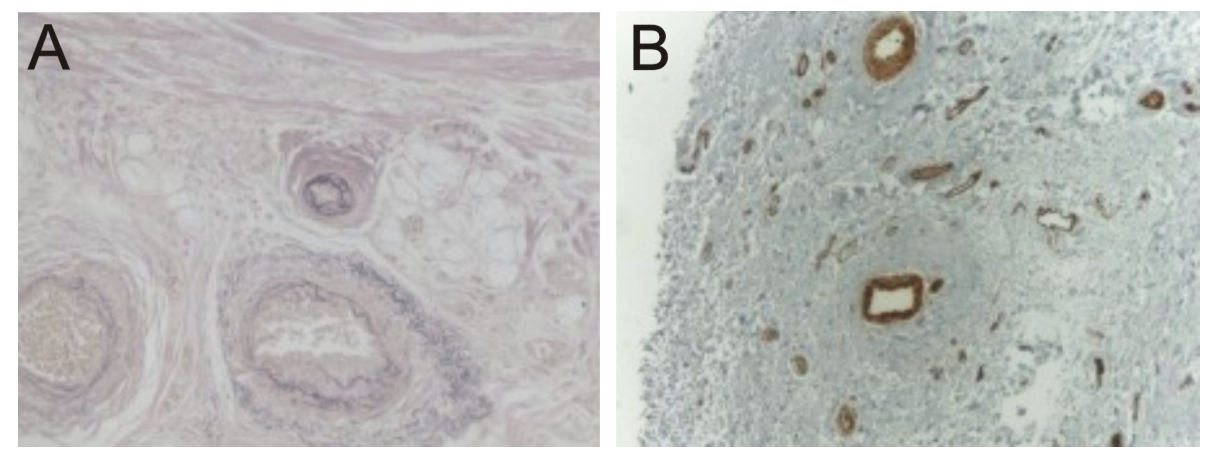

Abbildung 3.44: A feine elastische Fasern um die Kapillaren der Adventitia; EvG; Vergrößerung 200fach; Präparat 12; B Kapillaren in der Adventitia; SMA; Vergrößerung 100fach; Präparat 11

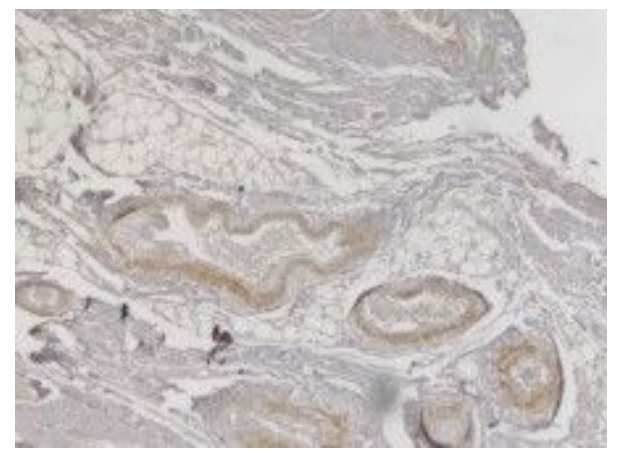

Abbildung 3.45: h-Caldesmon-positive Zellen in den Kapillarwänden der Adventitia; Vergrößerung 100fach; Präparat 12

wie CD68-Antigene wiesen T-Zellen bzw. Makrophagen nach. Es waren keine CD79-positiven B-Zellen oder CD138-positiven Plasmazellen in der Adventitia identifizierbar.

In der Adventitia des Präparats 13 überwogen die kräftigen Kollagen-IIIFasern gegenüber dem Subtyp I. Die Zellen zwischen den Fasern färbten hier positiv für Vimentin, lokalisiert zeigte sich eine positive Färbung für SMA. Die Adventitia enthielt viele inflammatorische Zellen, die ein perivaskuläres Infiltrat bildeten (Abb. 3.46).

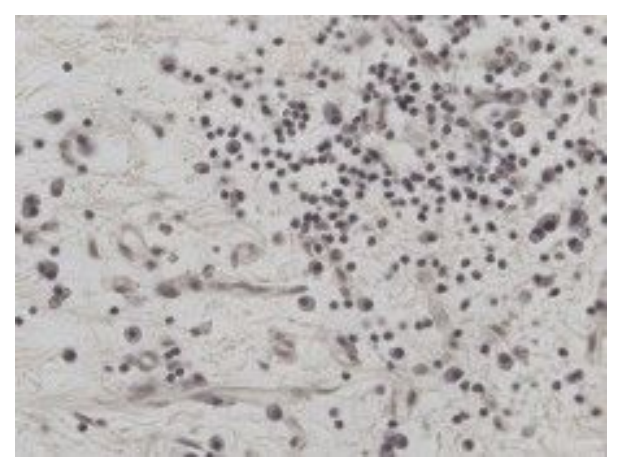

Abbildung 3.46: Lymphozyten-Infiltrat in der Adventitia; HE; Vergrößerung 400fach; Präparat 13 
CD3-positive T-Lymphozyten waren relativ gleichmäßig zwischen den Fasern verteilt. In dem perivaskulären Entzündungszell-Infiltrat fanden sie sich außerdem gruppiert. Unter den T-Lymphozyten konnten weiterhin CD8-positive Killerzellen und vergleichsweise zahlreich CD4-positive Helferzellen differenziert werden. Im Vergleich mit den anderen Schichten des Präparats zeigten sich in der Adventitia die meisten CD68-positiven Makrophagen. Darüber hinaus waren wenige CD79-positive B-Lymphozyten und außerdem CD138positive Plasmazellen nachweisbar.

Auch die Adventitia des Präparats 12 bestand aus dichten, gewellten elastischen und Kollagenfasern. Unter den kräftigen Kollagenfasern waren beide Subtypen vertreten. Insgesamt überwog Typ III. Die spindelförmigen Zellen zeigten eine positive Färbung für Vimentin, SMMy und h-Caldesmon. Die Adventitia enthielt viele Kapillaren und kleine Gefäße, große Ansammlungen von Erythrozyten sowie zahlreiche Fettzellen. Auch inflammatorische Zellen kamen vor und waren besonders perivaskulär lokalisiert (Abb. 3.47). Nach morphologischen Kriterien handelte es sich um Lymphozyten und Granulozyten. In den Färbungen mit den verwandten Antikörpern gegen die CD-Antigene 3, 68, 79 und 138 ließen sich keine spezifischen Zelltypen identifizieren.

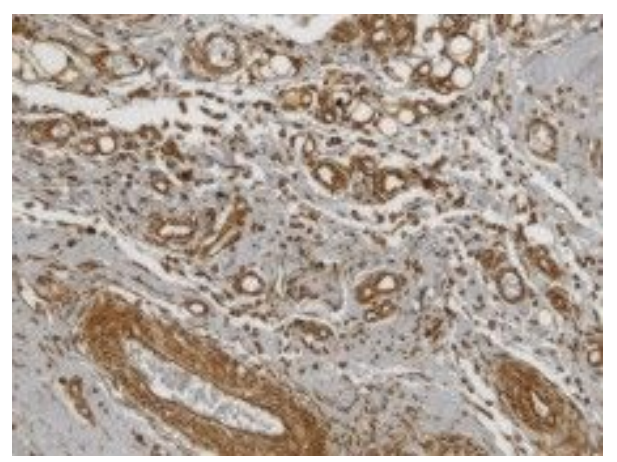

Abbildung 3.47: zahlreiche Kapillaren und Lymphozyten in der Adventitia; Vimentin; Vergrößerung 200fach; Präparat 12

\section{Klappen}

Im Präparat 11 war eines der ursprünglich drei Klappensegel der Venenklappe erhalten, von den beiden anderen waren makroskopisch nur noch fibröse Ansatzlinien in der Conduit-Wand sichtbar. Der erhaltene Klappenanteil war in der haptischen Untersuchung von derb-brüchiger Konsistenz und wies eine ungleichmäßige weißlich-gelbliche Oberfläche auf. Histomorphologisch hatte die Klappe als Grundgerüst die gewellten Fasern des Grafts. Sie lagen dicht aneinander. In der Pikrosirius-Rot-Färbung zeigten sich die Kollagen-Subtypen I und III (Abb. 3.48A). Das Klappensegel enthielt viel Fibrin. In der EvGFärbung ließen sich keine elastischen Fasern nachweisen. 

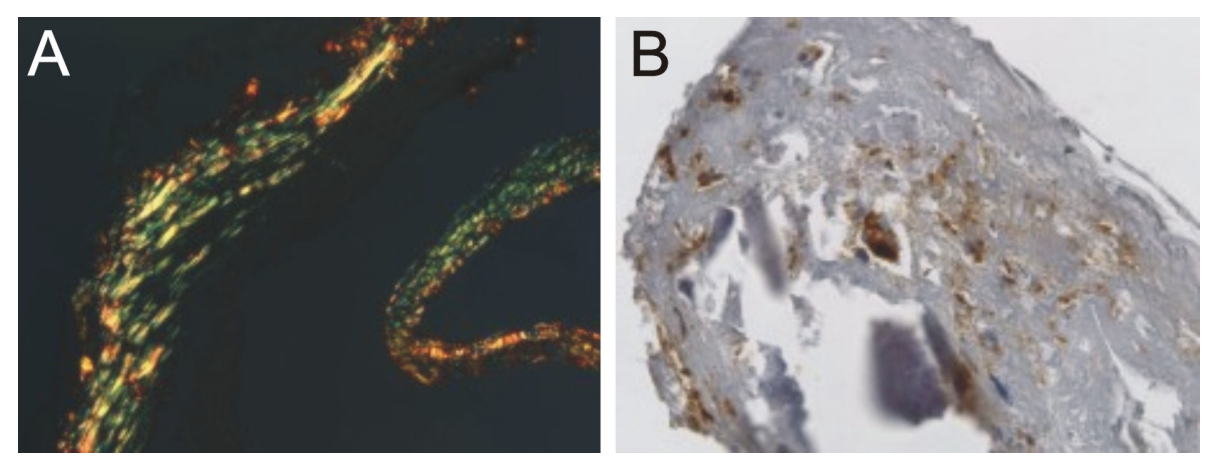

Abbildung 3.48: A Kollagenfasern im erhaltenen Klappensegel; Pikrosirius-Rot-Färbung; Vergrößerung 100fach; Präparat 11; B CD68-positive Fremdkörperriesenzellen im Klappensegel; Vergrößerung 400fach; Präparat 11

Zwischen den Fasern befanden sich zahlreiche runde und ovale Zellen mit unterschiedlich geformten Zellkernen. Es zeigten sich inflammatorische Zellen. Hierbei handelte es sich um CD68-positive einzelne Histiozyten und mehrkernige Riesenzellen (Abb. 3.48B). Dagegen fanden sich sehr wenige CD3-positive T-Lymphozyten und es kamen nur äußerst wenige CD79-positive B-Zellen vor.

Auf dem Klappensegel befand sich fibrinreiches thrombotisches Pseudointimagewebe mit aufgelockerter Struktur und amorphen Anteilen. An mehreren Stellen fanden sich Kalkeinschlüsse sowohl in der Pseudointima als auch dem Graft-Gewebe der Klappe (Abb. 3.49).

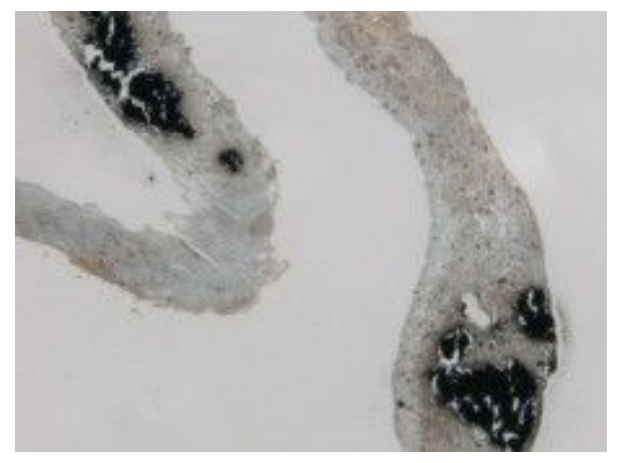

Abbildung 3.49: Kalkherde im Klappensegel; Färbung nach von Kossa; Vergrößerung 100fach; Präparat 11

Auf dem Klappensegel zeigte sich beidseits eine dünne von-WillebrandFaktor-positive Zelllage.

Ein Teil der fibrösen Grundstruktur des Klappen-Halteapparates war in der Conduit-Wand zwischen den Klappenabgängen erkennbar (Abb. 3.50A). Er enthielt unter anderem gewellte elastische Fasern (Abb. 3.50B). 
Die beiden einzelnen erhaltenen Klappensegel des Präparates 12 bestanden ebenfalls aus dichten gewellten Fasern, unter denen im Gegensatz zu Präparat 11 in der EvG-Färbung auch kurze elastische Fasern identifizierbar waren (Abb. 3.51). Es waren relativ wenige Zellen enthalten. Diese waren klein und länglich-oval bis länglich geformt.
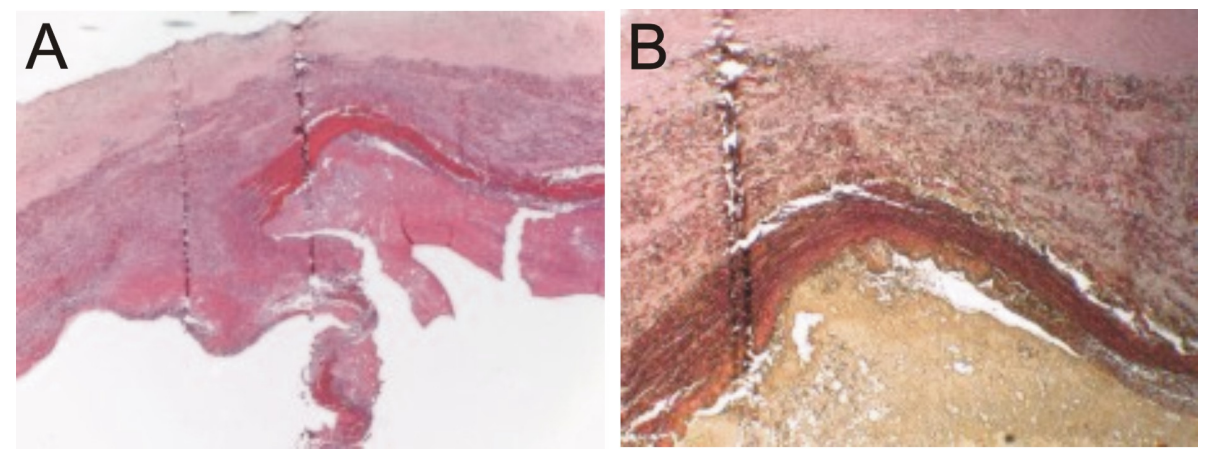

Abbildung 3.50: A fibröses Klappengerüst in der Conduit-Wand und Klappenursprung; HE; Vergrößerung 16fach; Präparat 11; B elastische Fasern im Klappengerüst; lumenseitig amorphe Substanz der thrombotischen Pseudointima zwischen den Klappenursprüngen; EvG; Vergrößerung 400fach; Präparat 11

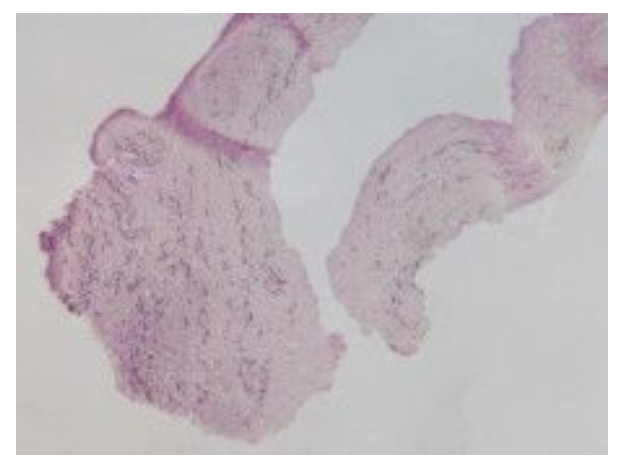

Abbildung 3.51: kurze elastische Fasern in den Klappensegeln; EvG; Vergrößerung 100fach; Präparat 12 


\subsection{PTFE-Conduits mit und ohne Schweineklappe}

In der Gruppe der Conduits aus synthetischem Material wurden zehn HancockConduits und ein klappenloser PTFE-Conduit untersucht. Ihre Implantationszeiten lagen zwischen zehn Monaten und 15 Jahren und acht Monaten. Der Grund für die Explantation war bei vier Patienten eine Stenosierung des Conduits, einmal bestand eine isolierte Insuffizienz und in sechs Fällen lagen sowohl eine Stenose als auch eine Insuffizienz vor.

\subsubsection{Histopathologische Auswertung der PTFE-Conduits}

\section{Pseudointima und Endothel}

In allen untersuchten Präparaten befand sich lumenseitig entlang der ConduitWand und an den Klappensegeln Pseudointima-Gewebe. Dieses war meist sowohl zirkulär als auch im longitudinalen Verlauf der Conduits unterschiedlich ausgeprägt. Mehrfach zeigte sich auch im Verlauf der Conduits (außerhalb der Klappenebene) eine verdickte Pseudointima, die in einem Präparat bis zu 3,8 mm dick war (Abb. 3.52).
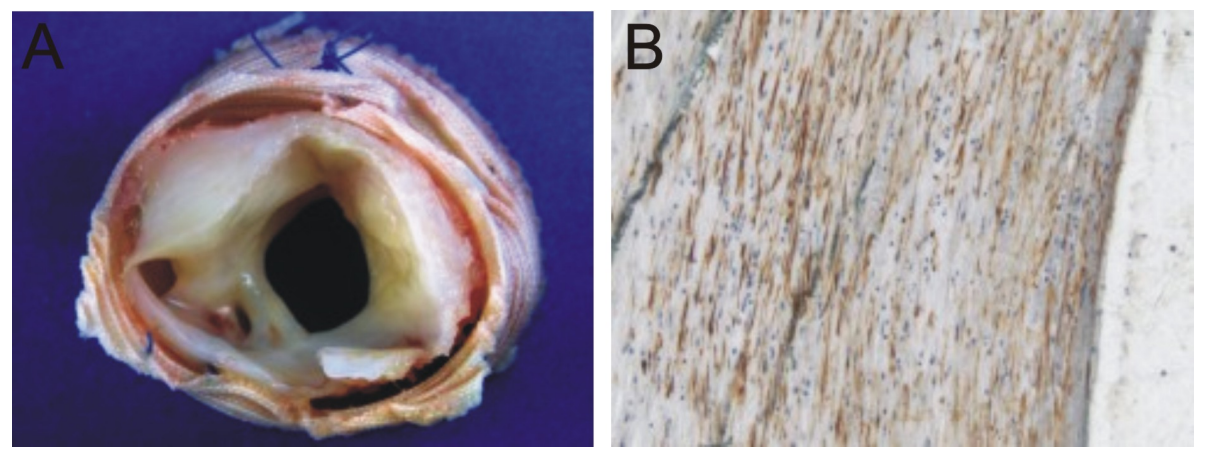

Abbildung 3.52: A verdickte Pseudointima außerhalb der Klappenebene; Präparat 24 nach Zuschnitt; B Pseudointima; SMA; Vergrößerung 200fach; Präparat 23

In der histologischen Untersuchung fanden sich in allen Conduits Abschnitte der Pseudointima, in denen sie bindegewebig organisiert war und aus dichten Fasern und spindelförmigen Zellen bestand. Diese Zellen färbten positiv für SMA und Vimentin sowie meist auch für SMMy (Abb. 3.52B). In jeweils vier Präparaten befanden sich zudem h-Caldesmon- und Desmin-positive Zellen in der Pseudointima.

Der klappenlose Conduit Nummer 15, der für 15 Jahre und acht Monate implantiert gewesen war, wies im Vergleich zu den übrigen eine relativ gleichmäßige Verteilung der Pseudointima auf (Abb. 3.53A). Die spindelförmigen Zellen färbten hier positiv für SMA, SMMy, h-Caldesmon, Desmin und Vimentin (Abb. 3.53B).

Die Pikrosirius-Rot-Färbung des klappenlosen PTFE-Conduits zeigte dichte Kollagenfasern sowohl des Subtyps I als auch III. In diesem und zwei weiteren Präparaten konnten feine elastische Fasern nachgewiesen werden, die sich vor allem in Lumen-Nähe befanden. In diesen fibrösen Arealen der Pseudointima war kein Fibrin enthalten. 

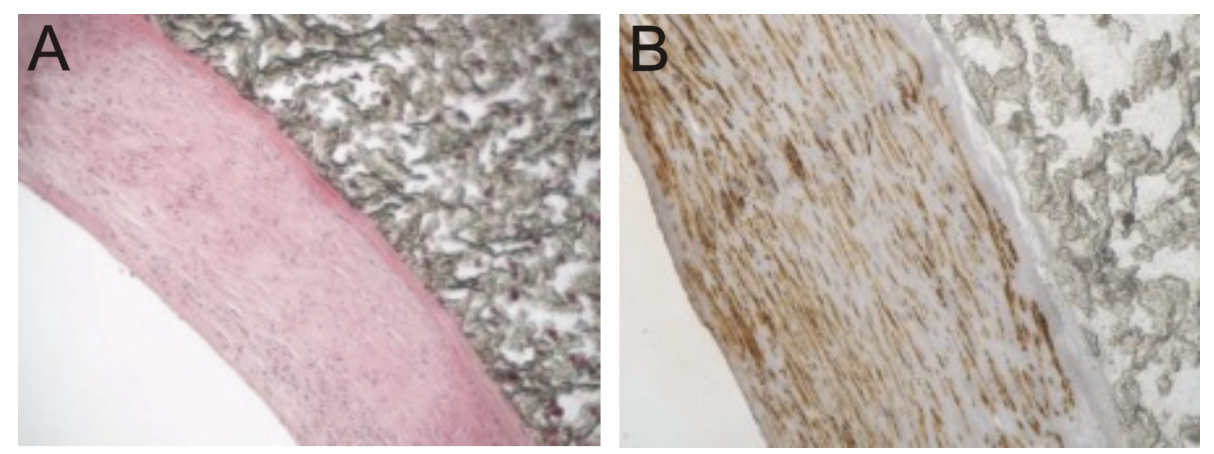

Abbildung 3.53: A Pseudointima und PTFE; HE; Vergrößerung 100fach; Präparat 15; B Pseudointima und PTFE; SMA; Vergrößerung 200fach; Präparat 15

Neben den beschriebenen zellulär organisierten Abschnitten wies die Pseudointima in allen Conduits auch septierte Bereiche auf (Abb. 3.54A). Zwischen den Septen fanden sich in unterschiedlicher Zahl Entzündungszellen und Erythrozyten.
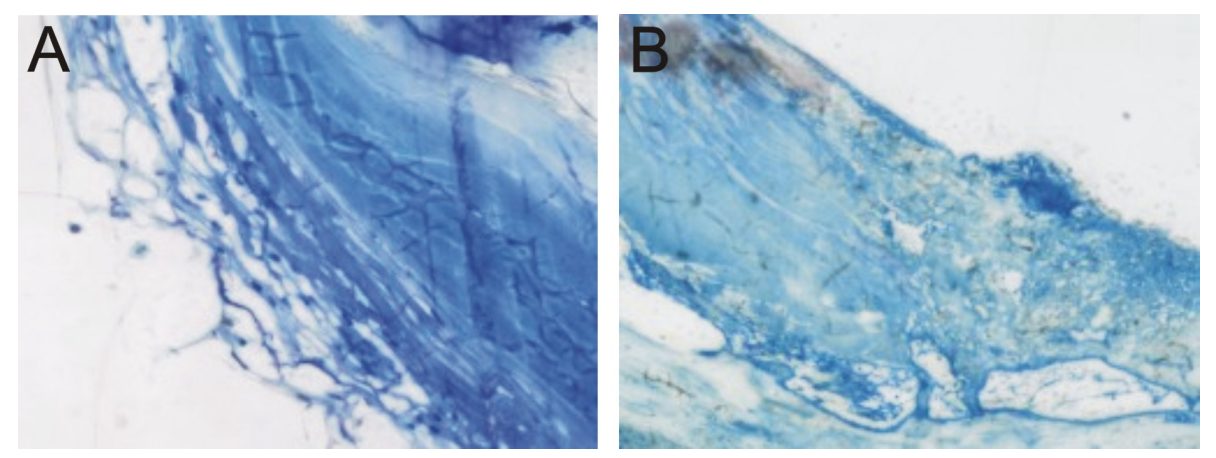

Abbildung 3.54: A septierte Pseudointima; Färbung nach Richardson; Vergrößerung 400fach; Präparat 17; B thrombotische Abschnitte der Pseudointima; Färbung nach Richardson; Vergrößerung 100fach; Präparat 18

An einigen Stellen war zudem amorphe (zellkernlose) Matrix enthalten. Die Pseudointima wies dort also eine thrombotische Struktur auf (Abb. 3.54B). Die organisierten Abschnitte der Pseudointima befanden sich vor allem entlang der zirkulären Conduit-Wand. Sehr ausgeprägt zeigte sich das septierte und thrombotische Gewebe in den Klappensinus, in den Winkeln zwischen Klappe und Conduit oder in Einfaltungen der Wand (Abb. 3.55A). Es kamen hier zum Teil zahlreich Granulozyten, Lymphozyten und Erythrozyten vor (Abb. 3.55B).

Darüber hinaus fand sich thrombotische Pseudointima vielfach in der Nähe des Graft-Gewebes bzw. zwischen der PTFE-Membran und dem SchweineGewebe im klappentragenden Segment (Abb. 3.56A). 

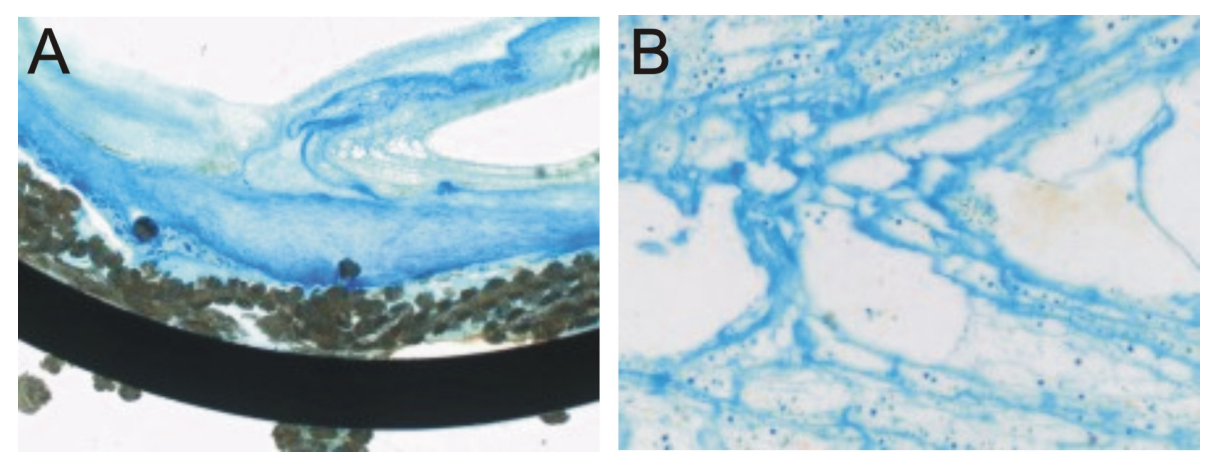

Abbildung 3.55: A septierte Pseudointima im Winkel zwischen Klappe und Conduit-Wand; Färbung nach Richardson; Vergrößerung 16fach; Präparat 23; B Vergrößerung aus A: Entzündungszellen und Erythrozyten zwischen den Septen; Färbung nach Richardson; Vergrößerung 200fach; Präparat 23
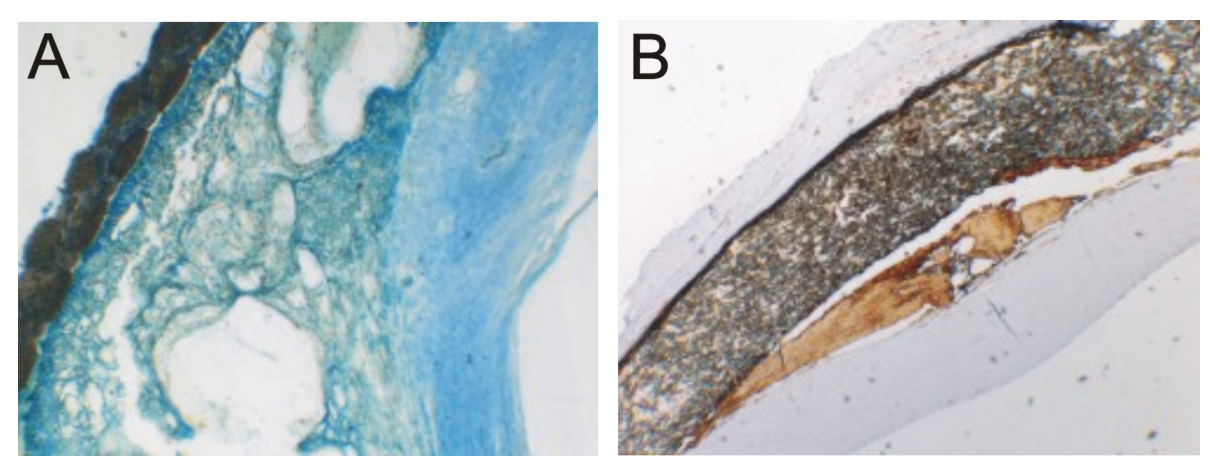

Abbildung 3.56: A thrombotisches Pseudointima-Gewebe nahe der PTFE-Membran; Färbung nach Richardson; Vergrößerung 40fach; Präparat 19; B fibrinreiche thrombotische Pseudointima; Fibrinfärbung; Vergrößerung 40fach; Präparat 15

Der klappenlose PTFE-Conduit (15) enthielt kein Fibrin in der bindegewebig organisierten Pseudointima. Dagegen färbte ein abgegrenztes Areal thrombotischer Pseudointima zwischen organisierter Pseudontima und den PTFEFasern deutlich positiv für Fibrin (Abb. 3.56B).

Lumenseitig ließ sich in den Conduits eine einlagige Endothelzellschicht erkennen, die positiv für von-Willebrand-Faktor und für Vimentin färbte (Abb. $3.57 \mathrm{~A})$.

In allen Hancock-Conduits enthielt das Pseudointima-Gewebe Kapillaren. Deren innere Zelllage war üblicherweise von-Willebrand-Faktor-positiv. Die Zellen der Kapillarwände färbten häufig positiv für SMA sowie gelegentlich auch für SMMy, h-Caldesmon und Vimentin. In mehreren Präparaten waren die Kapillaren gehäuft im äußeren, dem Graft benachbarten Teil der Pseudointima lokalisiert (Abb. 3.57B). Auch im Pseudointima-Gewebe zwischen PTFE und porcinem Segment zeigten sich Kapillaren. Außer Gefäßen waren in der Pseudointima einzelner Präparate Lakunen in einem ähnlichen Muster wie in den untersuchten Homografts zu finden. Im Gegensatz zu den HancockConduits waren in dem klappenlosen PTFE-Conduit keine Kapillaren enthalten. 

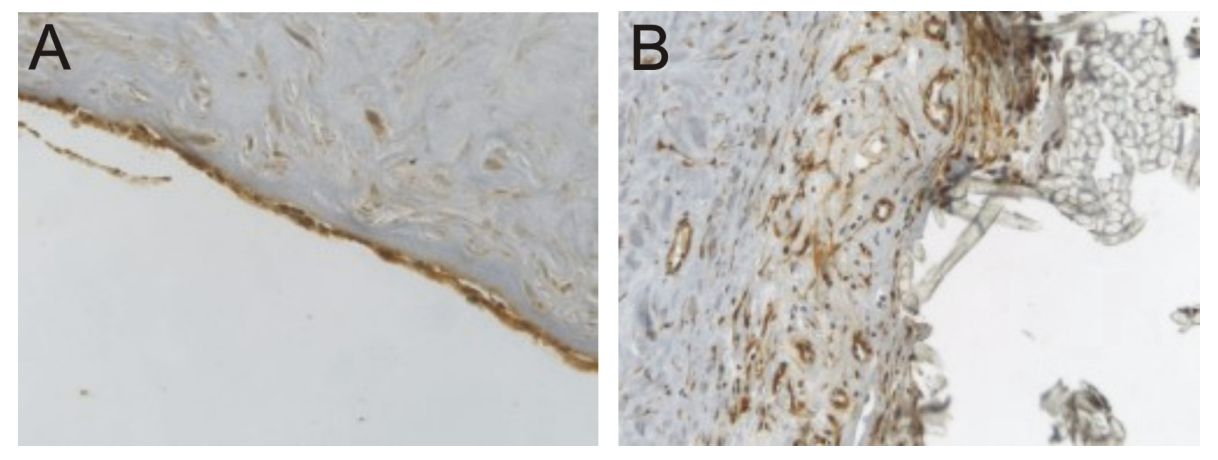

Abbildung 3.57: A lumenseitiges Endothel; von-Willebrand-Färbung; Vergrößerung 400fach; Präparat 14; B Kapillaren im zum Graft gewandten Teil der Pseudointima (Gewebe präparationsbedingt im Bereich des PTFE ausgerissen); SMA; Vergrößerung 200fach; Präparat 14

Fünf der untersuchten Conduits, deren Implantationszeiten jeweils deutlich über fünf Jahren lagen, enthielten Kalk in der Pseudointima. Hierbei handelte es sich um versprenkte kleinere Kalkareale. In einem Präparat ragten die Kalkherde zum Teil in das Lumen hinein vor und waren von Endothel bedeckt. Im Vergleich zu diesen kleineren Arealen fanden sich in der Pseudointima des klappenlosen PTFE-Conduits neben diffuser und intrazellulärer Verkalkung auch mehrere große Kalkeinschlüsse (Abb. 3.58).
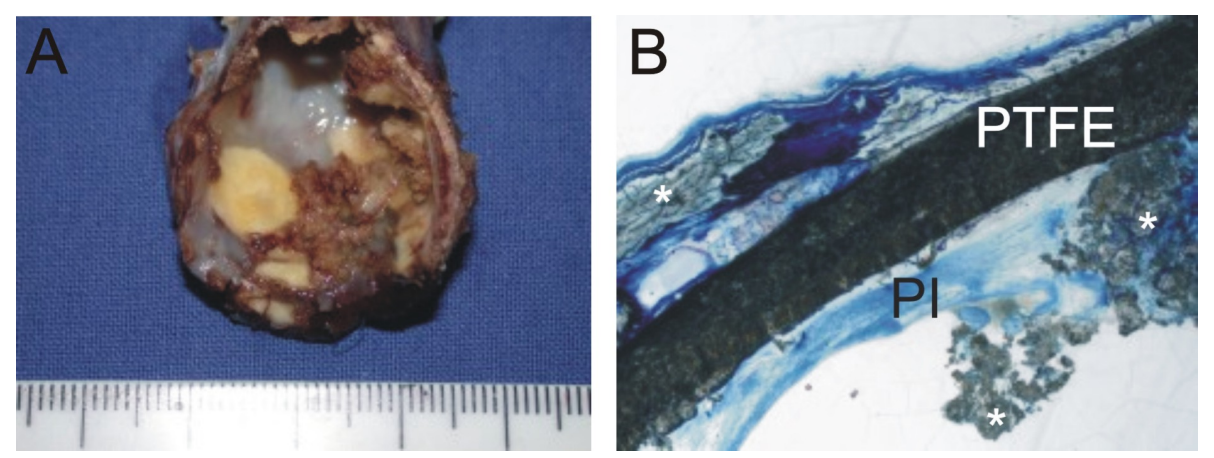

Abbildung 3.58: A typischer makroskopischer Aspekt flächiger Verkalkung; Präparat 15; B mikroskopischer Befund deutlicher, schichtübergreifender Verkalkung $\left(^{*}\right)$; Färbung nach Richardson; Vergrößerung 16fach; Präparat 15

Diese großen Kalkareale enthielten amorphe Substanz, Erythrozyten und wenige Entzündungszellen. Lumenwärts waren die Kalkherde von Pseudointima bedeckt, die sich aus spindelförmigen Zellen und Extrazellulärmatrix zusammensetzte.

In den untersuchten Hancock-Conduits zeigten sich in der Pseudointima schon in den konventionellen Färbungen große Ansammlungen von Lymphozyten, die in den meisten Fällen vor allem in Graft-Nähe lokalisiert waren. In einigen Präparaten fanden sich auch perivaskulär zahlreiche inflammatorische Zellen. Es konnten CD3-positive T-Lymphozyten und CD79-positive B-Lymphozyten identifiziert werden (Abb. 3.59 und 60). Beide Zellpopulationen kamen sowohl einzeln als auch gruppiert vor. Bei den T-Lymphozyten konnte zum Teil eine weitere Differenzierung in CD4-positive T-Helferzellen und CD8-positive 
T-Killerzellen vorgenommen werden. Dabei zeigten sich in zwei Präparaten vergleichsweise zahlreich einzeln liegende CD8-positive Zellen sowie in einem außerdem wenige CD4-positive T-Lymphozyten. Beide Zelltypen befanden sich vermehrt in der Nähe des Graft-Gewebes. Auch CD68-positive Makrophagen kamen vor und zeigten sich als einzelne Histiozyten sowie als mehrkernige Riesenzellen. Sie fanden sich ebenfalls gehäuft dem Graft-Gewebe benachbart.
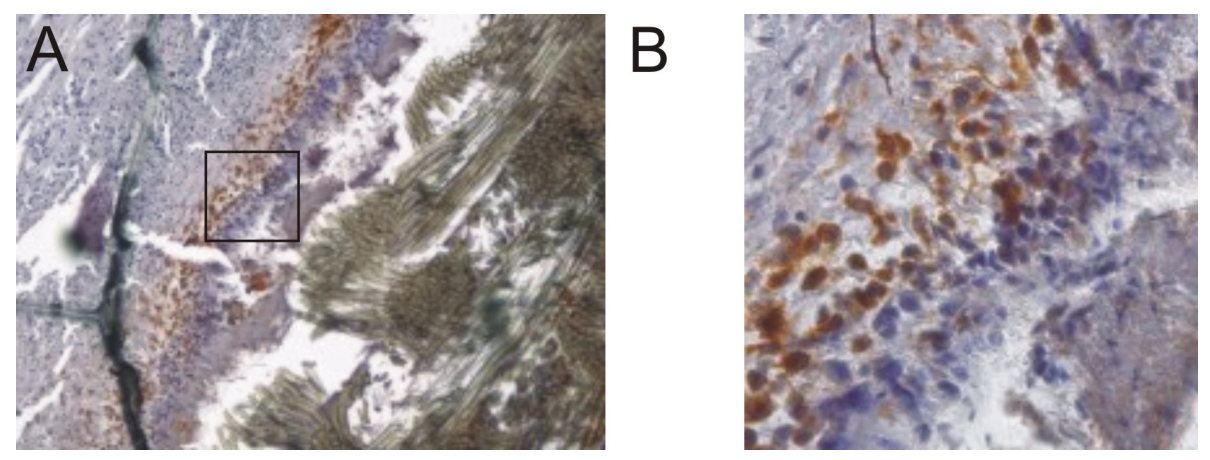

Abbildung 3.59: A ausgedehnte Lymphozyten-Infiltrate in PTFE-Nähe in der Pseudointima; Zellen positiv für CD79 (siehe Bild) sowie CD3 und CD8; CD79; Vergrößerung 100fach; Präparat 21; B B-Zellen aus A; CD79; Vergrößerung 400fach; Präparat 21

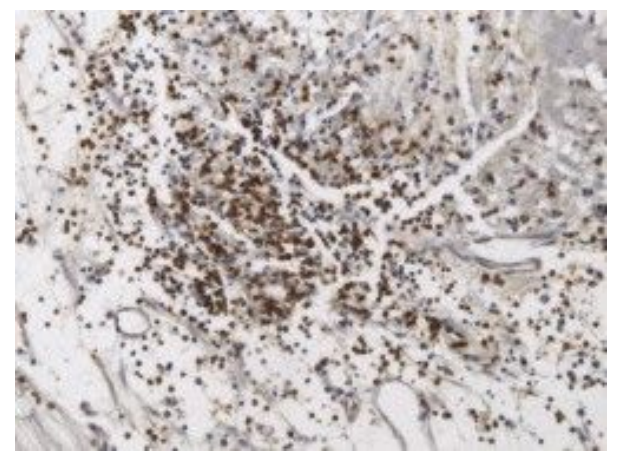

Abbildung 3.60: T-Lymphozyten perivaskulär in der Pseudointima; CD3; Vergrößerung 200fach; Präparat 18

Im Präparat 14, das bei bakterieller Endokarditis des Patienten explantiert worden war, war die Pseudointima vielfach septiert und wies thrombotische Abschnitte auf. Sie enthielt vor allem lumenseitig massenhaft Granulozyten und Lymphozyten sowie Erythrozyten. Dieses Bild bot sich auch an den Klappensegeln und besonders ausgeprägt in den Klappensinus (Abb. 3.61). Intraoperativ hatten sich makroskopisch im Bereich des klappentragenden Segments Eiter und infiziert wirkendes thrombotisches Material gezeigt. Unter den inflammatorischen Zellen in den septierten Bereichen ließen sich zahlreiche einzeln liegende CD3-positive T-Zellen identifizieren. Ein Nachweis anderer CD-Antigene gelang mit den verwendeten immunhistochemischen Färbungen nicht. In Teilen dieses Conduits, die außerhalb der Klappenebene lagen, waren nur noch vergleichsweise wenige inflammatorische Zellen in der Pseudointima zu finden (Abb. 3.62). 

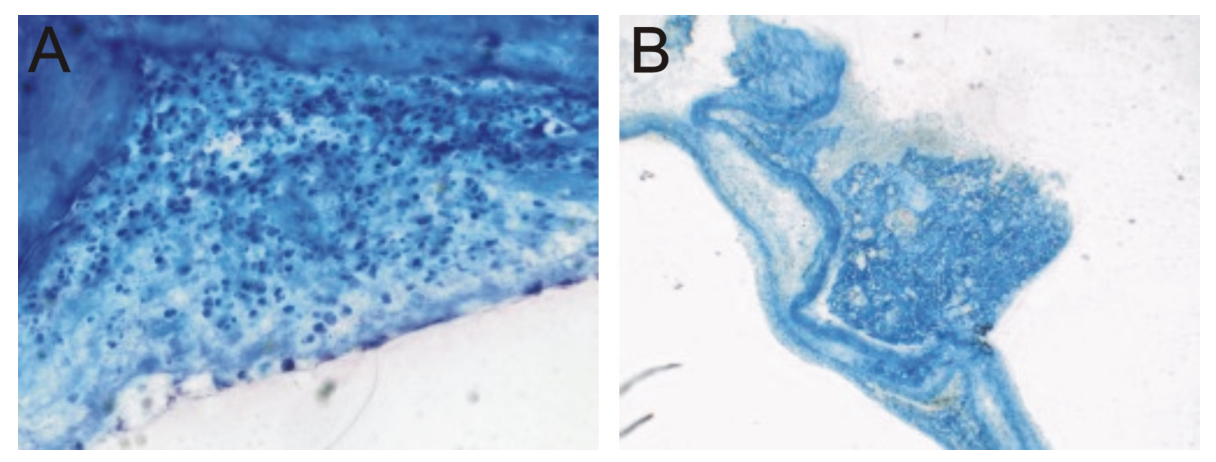

Abbildung 3.61: A Entzündungszellen und Erythrozyten in der Pseudointima (unten Lumen); Färbung nach Richardson; Vergrößerung 400fach; Präparat 14; B makroskopisch infiziert wirkendes thrombotisches Material an einem der Klappensegel; Färbung nach Richardson; Vergrößerung 40fach; Präparat 14

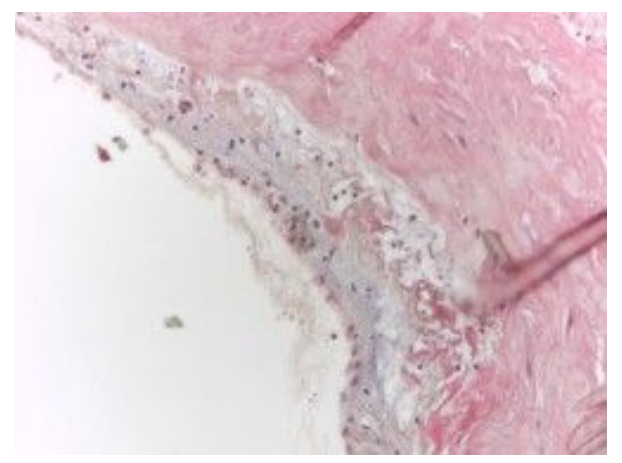

Abbildung 3.62: weiter proximal weniger Entzündungszellen in der septierten Pseudointima; HE; Vergrößerung 200fach; Präparat 14

\section{Graft-Gewebe}

Das Graft-Gewebe der Hancock-Conduits bestand - wie oben beschrieben - aus einem PTFE-Schlauch, in den eine Schweine-Klappe an einem tragenden zylindrischen Segment aus porcinem Gewebe eingenäht war. Der außen befindliche Metallring war in mehreren Conduits auch im histologischen Präparat beurteilbar und stellte sich in allen Fällen unauffällig dar. Das Graft-Gewebe des klappenlosen Conduits bestand ausschließlich aus einer PTFE-Röhre. Bei dem klappentragenden Schweine-Gewebe handelte es sich um gefäßwandtypisches Bindegewebe, das sich aus dichten, leicht gewellten Fasern und spindelförmigen Zellen zusammensetzte (Abb. 3.63A). Von diesen färbten in einzelnen Präparaten Zellen positiv für Vimentin und SMA sowie vereinzelt für Desmin und h-Caldesmon.

Neun der zehn Hancock-Conduits wiesen im Schweine-Gewebe Kalkeinlagerungen auf. Hierbei handelte es sich sowohl um einzelne verkalkte Areale als auch mehrfach um flächige Verkalkung (Abb. 3.63B). In einigen Präparaten wirkte die Conduit-Wand auch makroskopisch verkalkt. Als einziger Conduit wies Nummer 20 nach einer Implantationszeit von lediglich zehn Monaten keine Verkalkung auf.

Nach außen hin schloss sich dem porcinen klappentragenden Segment PTFEMaterial an. Dieses war in allen untersuchten Präparaten zirkulär erhalten. Um 
die PTFE-Fasern herum befand sich unterschiedlich ausgeprägt PseudointimaGewebe. Der klappenlose Conduit war nach 15 Jahren und acht Monaten Implantationszeit erheblich verkalkt (Abb. 3.64). In der haptischen Untersuchung wies er eine hart-brüchige Konsistenz auf. Mikroskopisch ließen sich mehrere Areale flächiger Verkalkung erkennen, die vor allem in der Nähe der Adventitia lokalisiert waren. Außerdem fanden sich kleinere Kalkpartikel.
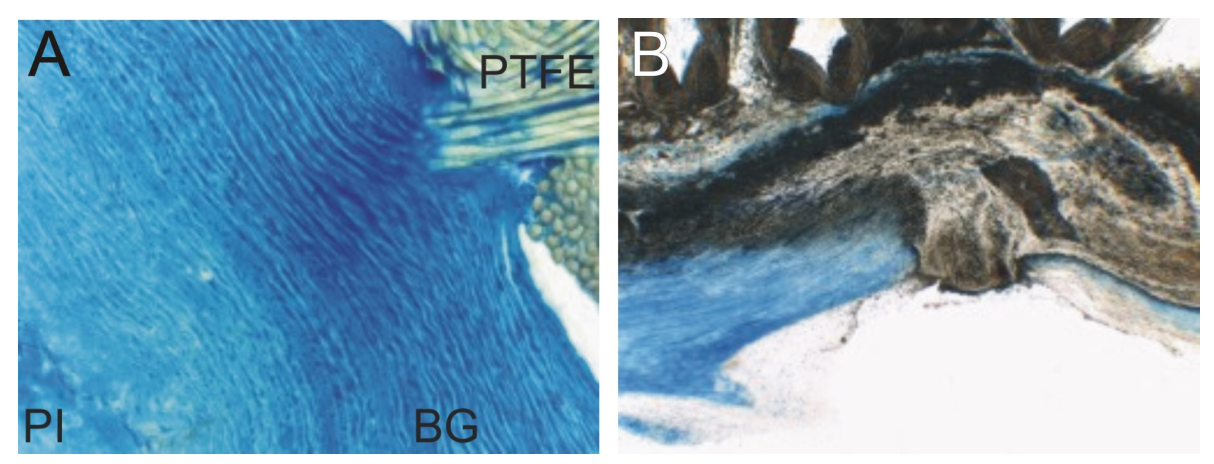

Abbildung 3.63: A Graft-Gewebe eines Hancock-Conduits aus PTFE-Material und dichtem Bindegewebe (BG) des porcinen klappentragenden Segments; links im Bild Anteile der Pseudointima (PI); Färbung nach Richardson; Vergrößerung 200fach; Präparat 14; B flächige Verkalkung im porcinen Gewebe (unten Lumen); Färbung nach Richardson; Vergrößerung 40fach; Präparat 17

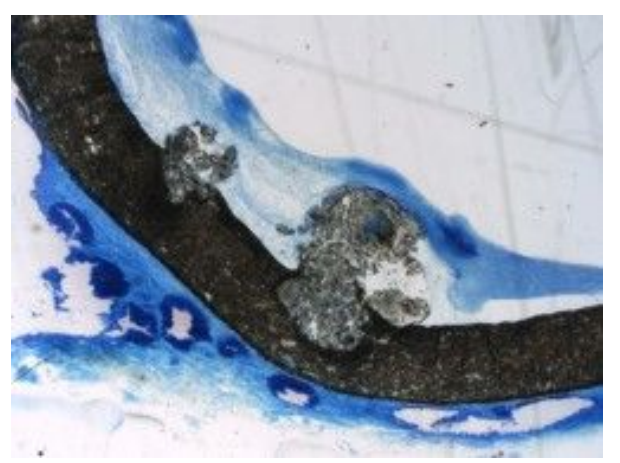

Abbildung 3.64: Verkalkung auch im PTFE-Gewebe; Färbung nach Richardson; Vergrößerung 16fach; Präparat 15

Generell zeigte sich in den Hancock-Conduits eine deutliche Entzündungsreaktion. Im Schweine-Gewebe konnten die inflammatorischen Zellen in sechs der zehn Conduits mit immunhistochemischen Färbungen klassifiziert werden. Es fanden sich in zwei Präparaten zahlreiche einzeln liegende CD3-positive T-Lymphozyten im gesamten porcinen Gewebe. Im Gegensatz hierzu kamen die Entzündungszellen in vier Präparaten ausschließlich im Randbereich des Schweine-Gewebes an der Grenze zur Pseudointima vor (Abb. 3.65). Hierbei handelte es sich in allen Fällen um CD68-positive Makrophagen, in drei Conduits zusätzlich um einzelne CD3-positive T-Zellen. 

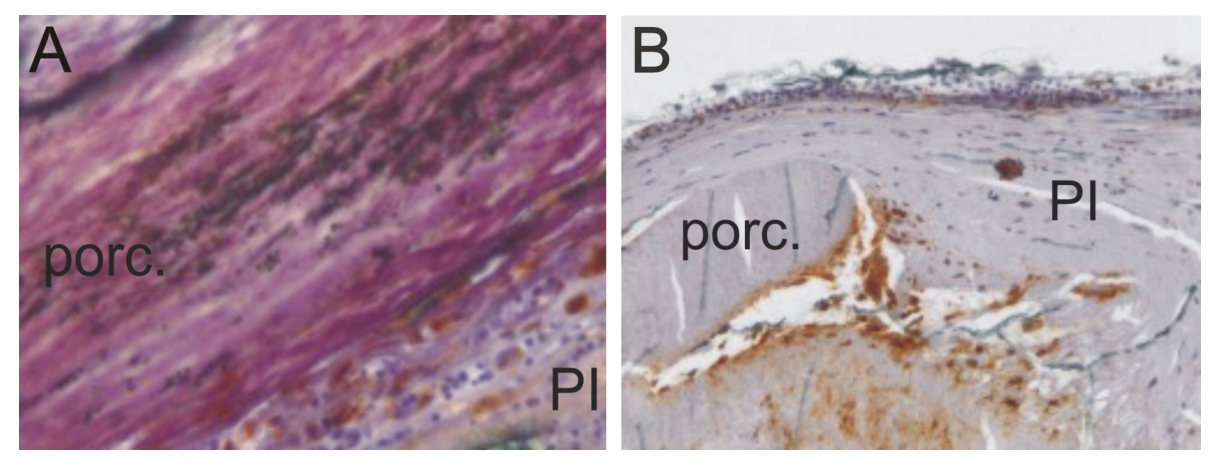

Abbildung 3.65: A einzeln liegende CD68-positive Makrophagen ausschließlich im Randbereich des Schweine-Gewebes; Vergrößerung 400fach; Präparat 23; B CD68-positive Fremdkörperriesenzellen im Randbereich des porcinen Gewebes (porc.) angrenzend an die Pseudointima (PI); Vergrößerung 200fach; Präparat 23

Mit der Färbung gegen CD8-Antigene konnten in zwei Präparaten reichlich einzelne CD8-positive T-Killerzellen nachgewiesen werden, in einem zusätzlich wenige einzelne CD4-positive T-Helferzellen. Darüber hinaus zeigten sich in zwei Präparaten CD79-positive B-Lymphozyten. Um die PTFE-Fasern herum ließen sich in allen Präparaten CD68-positive Makrophagen erkennen. Es fanden sich hierbei grundsätzlich einzelne Histiozyten und in den meisten Conduits auch mehrkernige Riesenzellen (Abb. 3.66).

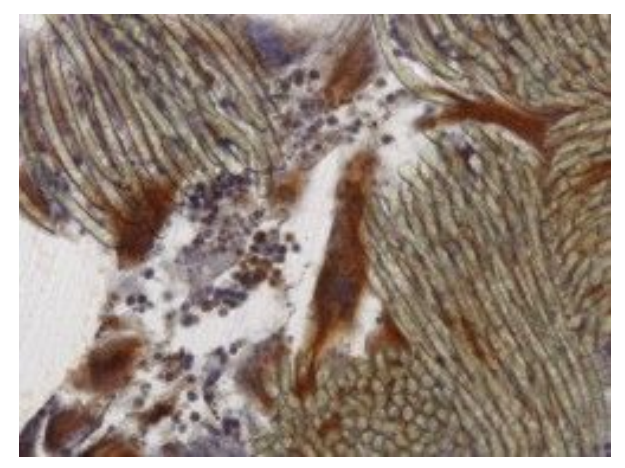

Abbildung 3.66: CD68-positive Histiozyten und mehrkernige Riesenzellen um die PTFEFasern; Vergrößerung 200fach; Präparat 20

Ebenso ließen sich zahlreiche CD3-positive T-Lymphozyten innerhalb des PTFE-Materials nachweisen. Sie kamen vorwiegend einzeln vor. CD79-positive B-Lymphozyten fanden sich im PTFE-Gewebe der Mehrzahl der untersuchten Conduits. Sie kamen einzeln und in einigen Fällen zusätzlich gruppiert liegend vor. Generell ließen sich in Graft-Nähe mehr T- als B-Lymphozyten erkennen. Im Vergleich zu den Hancock-Conduits waren im Graft-Gewebe des PTFEGrafts ohne Schweine-Gewebe nur wenige Entzündungszellen enthalten. 


\section{Adventitia}

Bei der Explantation der zehn Hancock-Conduits wurde der PTFE-Schlauch jeweils aus der Adventitia herausgelöst, während diese in situ belassen wurde. Dementsprechend ließ sich nur wenig Adventita-Gewebe mikroskopisch untersuchen. An den meisten Conduits waren außen am PTFE-Material bzw. zwischen Metallring und PTFE lediglich kleine Bereiche verblieben. Das AdventitiaGewebe bestand aus Fasern, amorpher Substanz sowie spindelförmigen und ovalen Zellen. Sie färbten in einem Präparat positiv für Vimentin. Zudem waren Entzündungszellen enthalten, von denen jeweils in einzelnen Präparaten Zellen positiv für CD3, CD8 und CD79 färbten (Abb. 3.67).
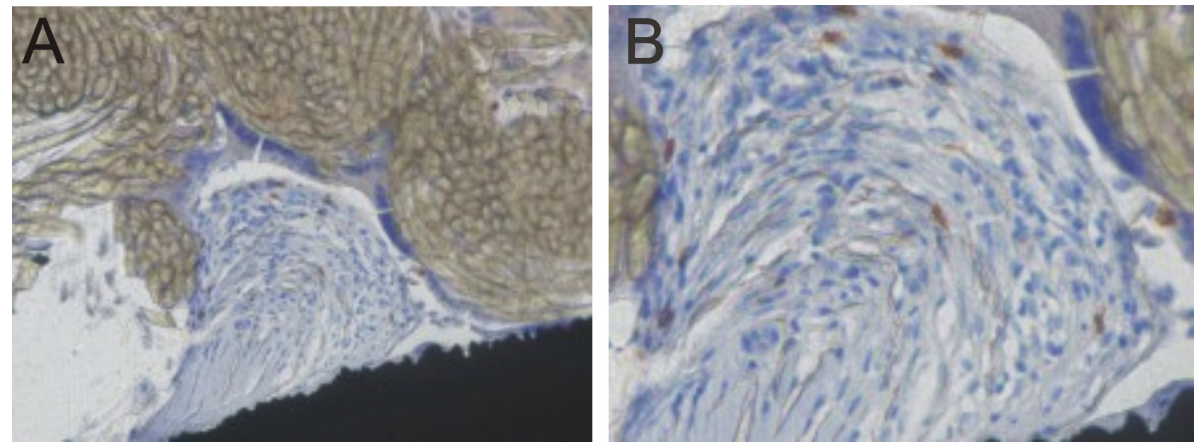

Abbildung 3.67: A CD8-positive T-Killerzellen im Adventitia-Gewebe zwischen PTFEMembran und Metallring; Vergrößerung 200fach; Präparat 18; B CD8-positive TKillerzellen, Ausschnitt aus A; Vergrößerung 400fach; Präparat 18

Außerdem fanden sich stellenweise kleine Kapillaren mit Erythrozyten sowie Fettzellen in der Adventitia. Der klappenlose PTFE-Conduit 15 sei nach einer Implantationszeit von 15 Jahren und acht Monaten laut OP-Bericht knöchern mit dem Sternum verbacken gewesen und habe flächenhafte Kalkeinlagerungen in der Adventitia gezeigt. Dieses Präparat war das einzige, in dem reichlich Adventitia-Gewebe erhalten war (Abb. 3.68).

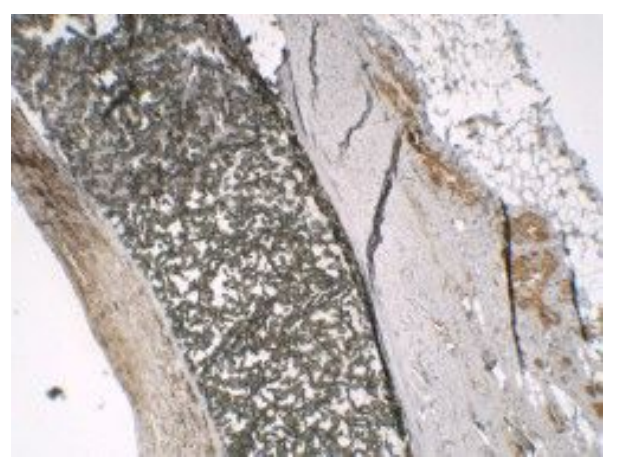

Abbildung 3.68: typischer Aspekt der Adventitia; SMA; Vergrößerung 40fach; Präparat 15

In der mikroskopischen Untersuchung wies es zirkulär um das PTFE-Material eine Schicht straffen Bindegewebes auf, das aus dichten gewellten Fasern und überwiegend spindelförmigen Zellen bestand. Unter den Fasern ließen sich in der Pikrosirius-Rot-Färbung Kollagenfasern vom Typ I und III nachweisen. In der EvG-Färbung zeigten sich feine stark gewellte elastische Fasern in der 
Adventitia. Die Zellen färbten an einer Stelle in dem straffen Bindegewebe positiv für SMA, SMMy, h-Caldesmon und Desmin und außerdem in der gesamten Adventitia positiv für Vimentin. Dem straffen Bindegewebe anliegend fand sich eine Schicht lockeren Bindegewebes. Dieses enthielt Muskelfaser-Bündel, Fettzellen, Kapillaren und kleine Gefäße sowie extravasale Ansammlungen von Erythrozyten. Die Zellen der Gefäßwände färbten positiv für SMA, SMMy, Vimentin, h-Caldesmon und Desmin. Ihre innere Zelllage war positiv für von-Willebrand-Faktor und Vimentin. In der mikroskopischen Untersuchung bestätigte sich eine deutliche Verkalkung, die sowohl flächig als auch in Form kleinerer Kalkpartikel vorlag. In den Färbungen für CD-Antigene zeigten sich in der Adventitia einzelne CD3-positive T-Lymphozyten, die an einer Stelle perivaskulär gruppiert vorkamen. Einzelne Lymphozyten waren CD8-positiv. CD68-positive Histiozyten zeigten sich in der gesamten Adventitia und vermehrt in PTFE-Nähe. In der Färbung für CD79alpha ließen sich wenige einzelne B-Lymphozyten nachweisen, während in der nicht-human-spezifischen Färbung nur sehr vereinzelt CD79-positive B-Zellen zu erkennen waren. Es fanden sich einige CD138-positive Plasmazellen in der Adventitia.

\section{Klappen}

Das Grundgerüst der aus drei Segeln bestehenden Taschenklappe wurde durch porcines Bindegewebe gebildet, das sich von der Conduit-Wand in die Klappe fortsetzte. Es bestand - wie in der Wand auch - aus dichten, leicht gewellten Fasern und länglichen Zellen. In der Mehrzahl der Präparate zeigte sich lumenseitig zu beiden Seiten der Klappe Pseudointima-Gewebe. Dieses wies an den Klappensegeln meist eine thrombotische Struktur aus Septen mit darin enthaltener amorpher Substanz auf (Abb. 3.69A).
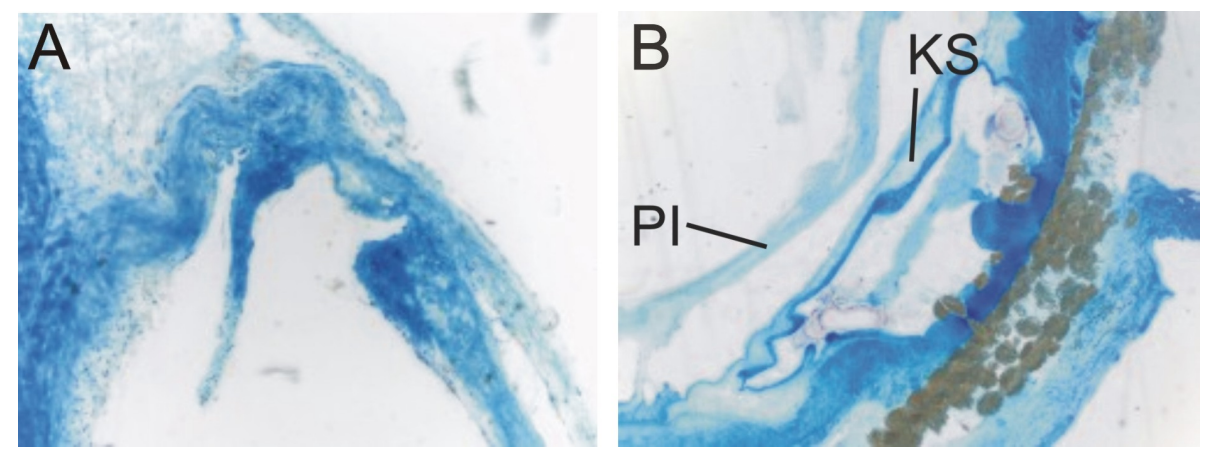

Abbildung 3.69: A Schweine-Gewebe des Klappensegels mit thrombotischer Pseudointima; Färbung nach Richardson; Vergrößerung 100fach; Präparat 16; B durch Pseudointima (PI) überdecktes Klappensegel (KS); Färbung nach Richardson; Vergrößerung 16fach; Präparat 20

Stellenweise wies die Pseudointima-Schicht auch die oben beschriebene bindegewebig organisierte Struktur mit Fasern und spindelförmigen Zellen auf. Diese Zellen färbten vereinzelt positiv für SMA und Vimentin. In drei Conduits waren zwei der drei Segel lumenseitig von einer kräftigen Lage PseudointimaGewebes bedeckt, so dass sie bei der Explantation laut OP-Bericht makroskopisch nicht mehr oder nur teilweise identifizierbar gewesen seien (Abb. 3.69B). 
Funktionell bestand eine Klappen-Insuffizienz.

Im Conduit Nummer 21 war das dritte Segel etwa zur Hälfte bedeckt und ragte mittig noch in das Lumen vor (Abb. 3.70A). Auch an diesem freien Abschnitt befand sich beidseits eine schmale Schicht Pseudointima-Gewebes (Abb. 3.70B).
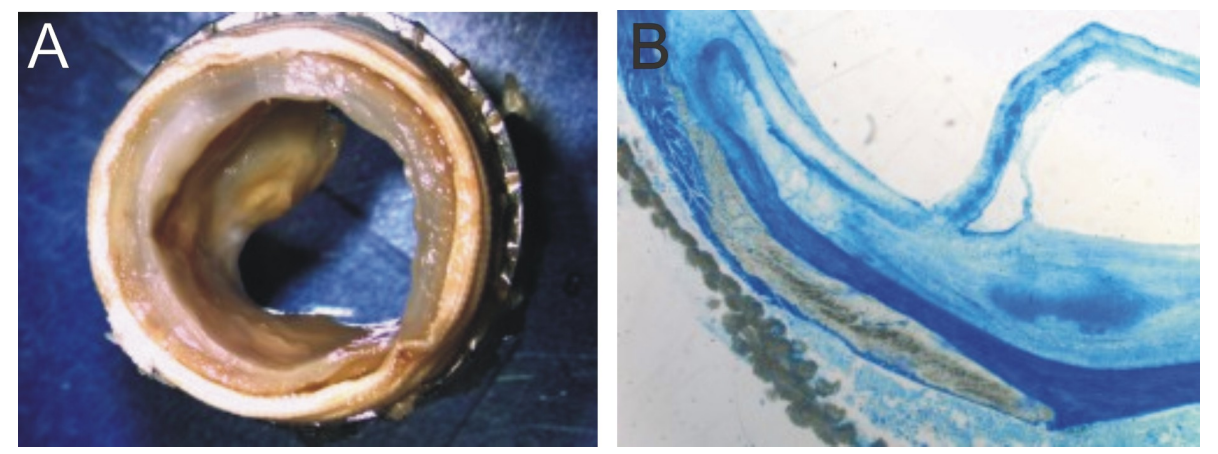

Abbildung 3.70: A Präparat 21 nach Zuschnitt; B ein Teil des Klappensegels bedeckt und an der Pseudointima im Klappensinus haftend; Färbung nach Richardson; Vergrößerung 16fach; Präparat 21

Im Präparat 23 befand sich an den Klappensegeln überwiegend septierte Pseudointima mit amorpher Substanz. Ein Segel war zum Teil von bindegewebig organisierter Pseudointima bedeckt, die von der Conduit-Wand in das Lumen hinein auf die Klappe zu verlief. Dadurch entstand im Schnitt das Bild einer Art Pseudointima-Brücke. Auch außerhalb der Klappenebene war diese noch im Lumen erkennbar (Abb. 3.71).
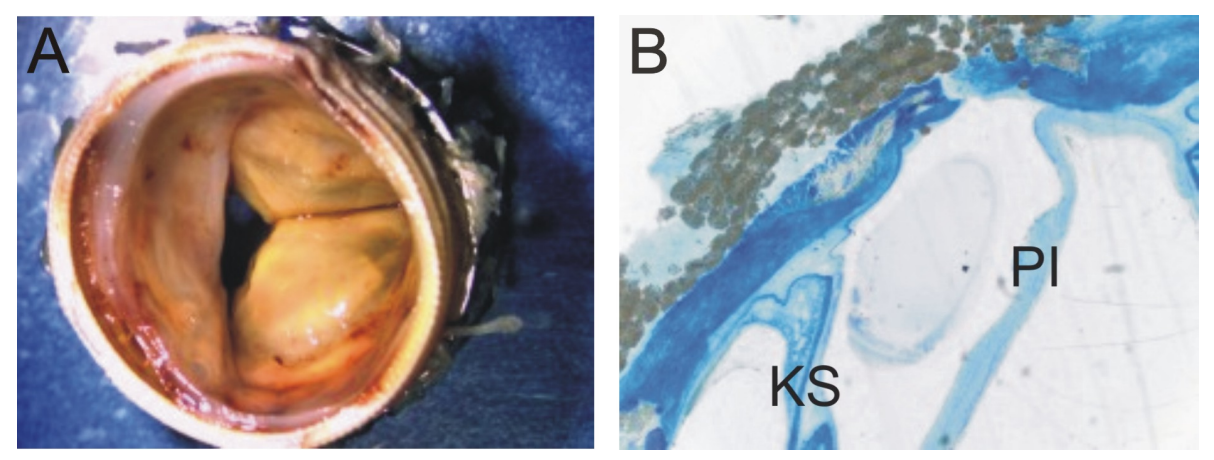

Abbildung 3.71: A ein Klappensegel makroskopisch durch Pseudointima verdeckt; Präparat 23; B Pseudointima-Brücke (PI) zwischen Conduit-Wand und Klappensegel (KS); Färbung nach Richardson; Vergrößerung 16fach; Präparat 23

Von den Präparaten mit freien Segeln zeigten mehrere auf der Klappe lumenseitig eine einlagige Schicht Endothelzellen (Abb. 3.72).

In den Klappen der untersuchten Präparate ließen sich Entzündungszellen erkennen. Es handelte sich um einzeln und gruppiert liegende CD3-positive TLymphozyten, CD68-positive Histiozyten und Fremdkörperriesenzellen sowie einzelne CD79-positive B-Zellen. In den meisten Präparaten fand sich in den Klappensinus Pseudointima-Gewebe. Dieses war vielfach septiert oder thrombotisch und enthielt in mehreren Fällen Kapillaren sowie inflammatorische 


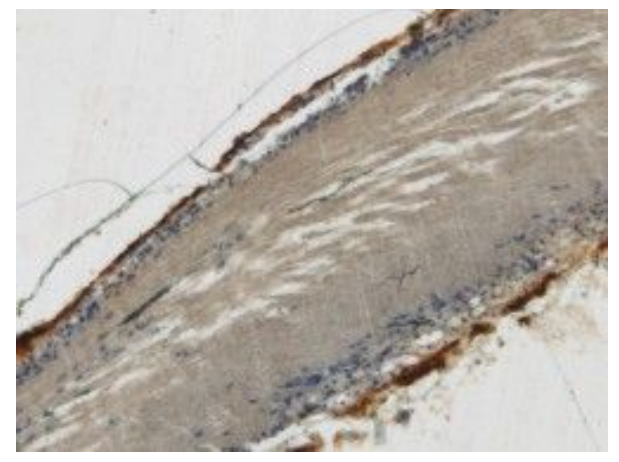

Abbildung 3.72: von-Willebrand-Faktor-positive Endothelschicht auf der Klappe; Vergrößerung 200fach; Präparat 23

Zellen und Erythrozyten (Abb. 3.73). In einigen Präparaten waren die Klappensinus beinahe vollständig mit Pseudointima-Gewebe ausgefüllt, das in diesen Fällen zu großen Teilen thrombotisch war.
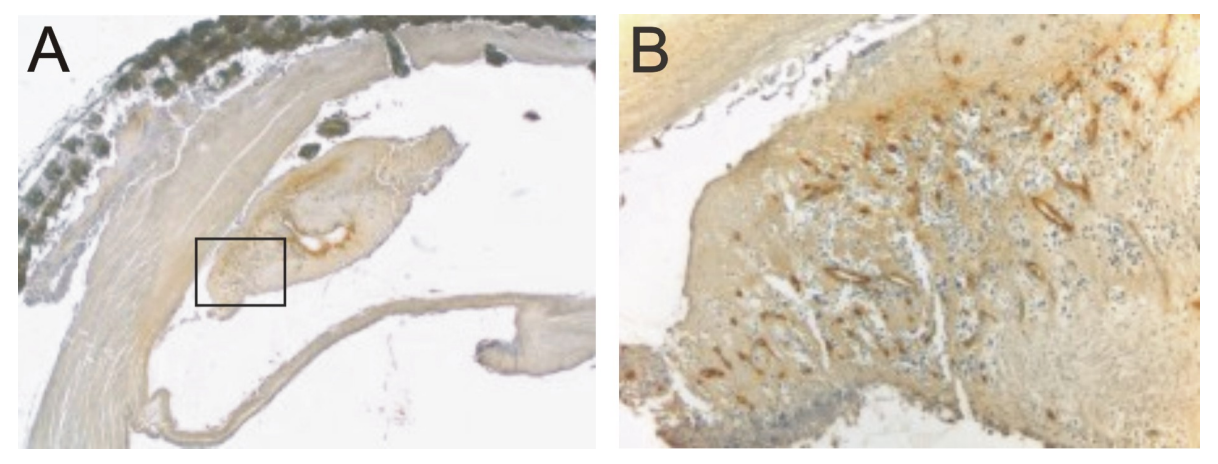

Abbildung 3.73: A Pseudointima-Gewebe im Klappensinus mit Kapillaren; von-WillebrandFärbung; Vergrößerung 16fach; Präparat 20; B Pseudointima-Gewebe im Klappensinus mit Kapillaren, vergrößerter Ausschnitt aus Abbildung A; von-Willebrand-Färbung; Vergrößerung 100fach; Präparat 20

In vier Präparaten ließen sich in der Klappe Verkalkungsareale erkennen, die mehrfach besonders die Klappenbasis betrafen (Abb. 3.74).

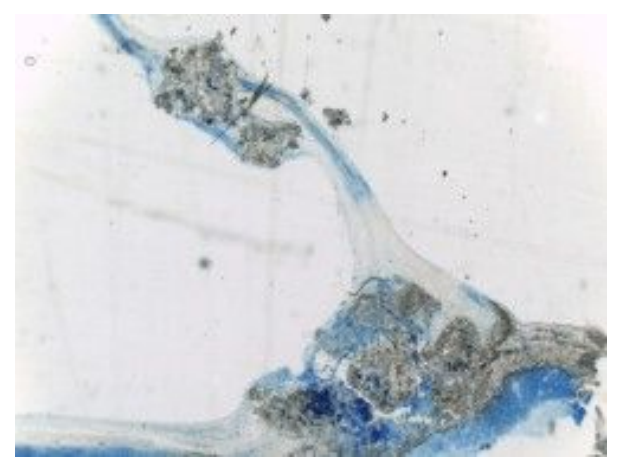

Abbildung 3.74: verkalktes Segel der insuffizienten Klappe; Färbung nach Richardson; Vergrößerung 16fach; Präparat 18 
Von der Klappe des Präparats 17 waren nur noch stark verkalkte Rudimente erkennbar (Abb. 3.75A). Diese wiesen eine sehr unebene Oberfläche auf, deren Einbuchtungen stellenweise septierte Pseudointima mit Erythrozyten und einigen inflammatorischen Zellen enthielten (Abb. 3.75B). Lumenseitig zeigte sich auch auf den Verkalkungsarealen eine einlagige Endothelschicht. In einigen Klappensegeln ließen sich Erythrozyten erkennen.
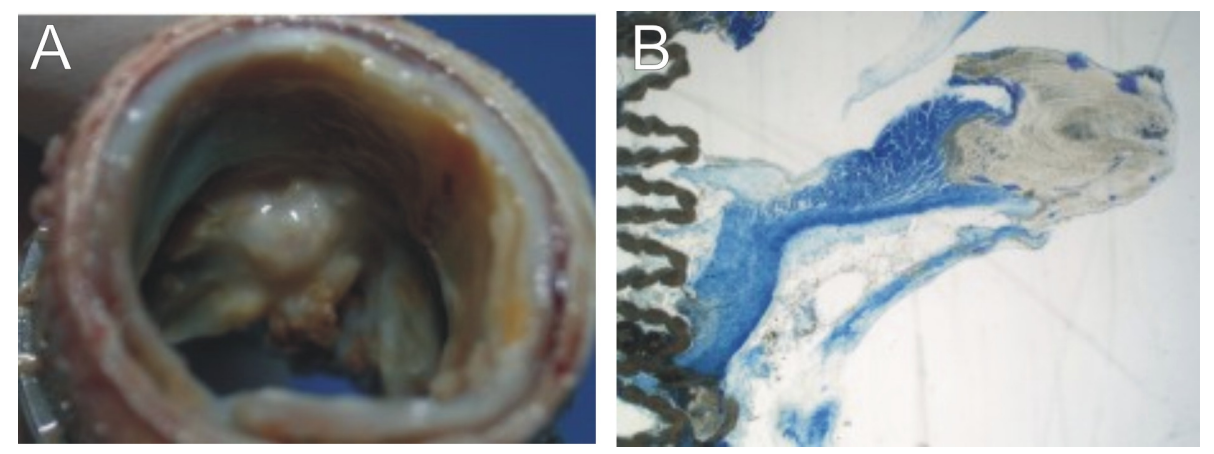

Abbildung 3.75: A Präparat 17 nach Zuschnitt; B verkalkte Klappenrudimente; Färbung nach Richardson; Vergrößerung 16fach; Präparat 17 


\section{Kapitel 4}

\section{Diskussion}

Während in der Literatur klinische Daten zu Conduits im rechtsventrikulären Ausflusstrakt vielfach analysiert wurden, sind nur wenige Studien über histologische Aspekte veröffentlicht worden, die die Biokompatibilität dieser Implantate betreffen. Die vorliegende Arbeit präsentiert die Ergebnisse der Auswertung von 24 histopathologisch und immunhistochemisch untersuchten Conduits, die an dieser Stelle mit Daten aus der Literatur in Bezug gesetzt werden.

\subsection{Methoden}

Da die in der vorliegenden Arbeit untersuchten Conduits vielfach Metallanteile sowie ausgeprägte Verkalkungen enthielten, konnten sie nur teilweise in Paraffin eingebettet werden. Anstatt dessen wurde vielfach als Einbettmedium das Kunstharz Methylmethacrylat verwandt. Dieses Verfahren ist hinsichtlich der Einbettung anderer Implantattypen in der Literatur gut beschrieben (Butany und Collins 2005, Farb et al. 2002, Kreischer 2008, Sigler et al. 2000, 2005 und Sigler und Jux 2007) und auch im Forschungslabor der Abteilung für Pädiatrische Kardiologie und Intensivmedizin der Universitätsmedizin Göttingen etabliert. Um an den in Methylmethacrylat eingebetteten Conduits immunhistochemische Färbungen vornehmen zu können, mussten die Präparate auf Glasobjektträgern fixiert und anschließend deplastifiziert werden, was technisch aufwendig ist. Im hiesigen Forschungslabor ist die Methode erfolgreich fortentwickelt worden und wird inzwischen routinemäßig angewandt (Quentin et al. 2009). Die humane Serie explantierter Conduits wurde für die vorliegende Arbeit nach einem einheitlichen Protokoll untersucht, das bereits in anderen Versuchsreihen angewandt wurde (Foth et al. 2009). Hierdurch war ein aussagekräftiger Vergleich zwischen den verschiedenen Präparaten möglich. Die Wichtigkeit eines strukturierten Vorgehens bei der pathologischen Analyse kardiovaskulärer Implantate wird auch in der Literatur betont (Butany und Collins 2005 und Schoen 1995).

Grundsätzlich sind bei histopathologischen Untersuchungen gewisse Limitationen zu beachten. Durch den teilweise schlechten Erhalt einiger Schnitte und vor allem der Schliffe ist die Aussagekraft bestimmter Befunde möglicherweise eingeschränkt. Nicht immer waren alle auszuwertenden Strukturen in jedem histologischen Schnitt enthalten (zum Beispiel Klappensegel). Hinzu kommt eine untersucherabhängige Subjektivität der Ergebnisse. In dieser rein deskriptiven 
Studie wurden histologische und immunhistochemische Methoden zur qualitativen Analyse der Conduits genutzt. Quantitative Auswertungen wurden hingegen nicht vorgenommen. So erfolgten beispielsweise kein Vergleich der Dichte inflammatorischer Zellen mit Daten anderer Quellen, keine quantitative Bestimmung des Calciumgehalts oder dergleichen.

\subsection{Endothelialisierung}

Erkenntnisse hinsichtlich Ablauf und Geschwindigkeit der (Re-)Endothelialisierung eines Conduits nach Implantation sind von unmittelbarer klinischer Relevanz: Die Gefahr für Thrombosen und Embolien ist umso geringer, je vollständiger die Endothelschicht auf den blutzugewandten Oberflächen ausgebildet ist (Pearson 1999). Aktuell wird Patienten in Göttingen sowie in den meisten anderen Zentren postoperativ eine sechsmonatige thrombozytenaggregationshemmende Therapie mit Acetylsalicylsäure (ASS) empfohlen. Dem liegt die Annahme zu Grunde, nach dieser Zeitspanne sei die Endothelialisierung abgeschlossen und damit das Risiko für thrombembolische Geschehen minimiert.

Interessant ist in diesem Zusammenhang, in wieweit das Endothel der Grafts vor Implantation erhalten ist. Goffin stellte an kryokonservierten, nicht-implantierten Klappen mit Antikörpern gegen Faktor VIII einen partiellen Erhalt des Endothels fest (Goffin 1997). Auch Fischlein et al. konstatierten einen Erhalt der Endothelzellen nach Kryokonservierung, sofern die Klappen innerhalb von 24 Stunden kryokonserviert worden waren (Fischlein et al. 1995). Andernfalls zeigten sich ein fortschreitender Verlust und eine Schädigung der Endothelzellen. Fischlein et al. wiesen nach, dass die erhaltenen Endothelzellen sowohl proliferationsfähig als auch metabolisch aktiv waren. Dagegen fanden Mitchell et al. an kryokonservierten und aufgetauten Homograft-Klappen vor Implantation kein Endothel (Mitchell et al. 1995). Armiger stellte fest, die Vorbehandlung von Homografts führe zu einer starken Reduktion der Zahl lebender Zellen (Armiger 1995). Lupinetti et al. zeigten mit Färbungen an Klappensegeln und Arterienwandanteilen von über 200 Homografts, dass nur an 16\% der kryokonservierten Präparate Endothelzellen nachweisbar waren (Lupinetti et al. 1993). Dagegen war das Endothel an 78\% der unbehandelten Grafts erhalten. Lupinetti et al. folgerten, dass Kryokonservierung üblicherweise zum Verlust des Endothels von Aorten- und Pulomonalis-Homografts führe. Koolbergen et al. beschrieben das Endothel an nicht-implantierten kryokonservierten Homograft-Klappen als stark beschädigt (Koolbergen et al. 2002a). Als Vorteil werteten Koolbergen et al., dass die Homografts durch den frühen Verlust des Endothels weniger immunogen seien. Andererseits könnten dadurch Proteine in das Gewebe eindringen. Diese führten zu Veränderungen der Extrazellulärmatrix und begünstigten somit beispielsweise die Entstehung von Atherosklerose.

Aus den widersprüchlichen Angaben der Literatur lässt sich die Schlussfolgerung ziehen, dass die Kryokonservierung von Conduits in variablem Ausmaß zu einem Erhalt lebender Spender-Endothelzellen führen kann. Hinsichtlich der Auswirkungen der Vorbehandlung von Xenografts mit Glutaraldehyd beschrieben Valente et al. einen resultierenden Verlust des Endothels (Valente 
et al. 1985). In dem für die vorliegende Arbeit untersuchten vorbehandelten, aber nicht implantierten Homograft war eindeutig eine Endothelschicht an der Conduit-Wand nachweisbar (vgl. Abb. 3.1B). Jedoch gehen wir davon aus, dass eventuell noch erhaltene Endothelzellen der Grafts nach der Implantation zu Grunde gehen. Diese These wird gestützt durch Ergebnisse anderer Gruppen, die innerhalb kurzer Implantationszeiten einen Endothelverlust nachwiesen. Goffin beschrieb an explantierten kryokonservierten Homografts einen frühen Verlust des Endothels beginnend wenige Stunden nach Implantation (Goffin 1997). Ab Tag 10 seien alle Endothelzellen zerstört gewesen. Als Ursache vermutete Goffin eine frühe humorale Immunantwort gegen die Conduits. $\mathrm{Hu}$ et al. wiesen nach, die Spender-Endothelzellen von arteriellen Grafts in Mäusen degenerierten innerhalb von vier Wochen (Hu et al. 2003).

Bei den für die vorliegende Arbeit untersuchten explantierten Conduits zeigte sich in der von-Willebrand-Färbung bei der Mehrzahl der Präparate unabhängig vom Grafttyp eine vollständige Endothelialisierung. Die Endothelzellen überzogen sowohl Conduit-Wand und Klappensegel als auch einen wandständigen Thrombus und Verkalkungsareale. Somit fanden sie sich an allen dem Blut zugewandten Flächen. Dies war auch bei dem Conduit mit der kürzesten Implantationszeit von sechs Monaten der Fall. Die Endothelialisierung der Implantatoberflächen wurde ebenso für andere bei Menschen eingesetzte kardiovaskuläre Implantate beschrieben. Hierbei finden sich unterschiedliche Angaben über die Zeitspanne bis zur Ausbildung einer vollständigen Endothelschicht. Sigler et al. stellten bei ihren Untersuchungen von PDA-Stents und PDA-Okkludern aus mehr als 200 Tieren sowie 49 aus dem menschlichen Kreislauf explantierten Implantaten unabhängig vom Implantattyp eine rasche (Re)Endothelialisierung der Oberflächen fest. Die Endothelschicht ließ sich sowohl elektronenmikroskopisch als auch immunhistochemisch durch Färbung mit Antikörpern gegen von-Willebrand-Faktor nachweisen (Sigler et al. 2005). Als ersten Schritt der Formierung der Zellschicht beschrieben Sigler et al. die Bildung eines Fibrinnetzes mit eingeschlossenen Blutzellen auf der blutzugewandten Oberfläche. Hieran schloss sich eine Ausbreitung von Endothelzellen auf dem Fibrinnetz frühestens ab Tag 10 post implantationem an. Eine intakte Schicht fand sich in allen explantierten Devices nach einer Implantationsdauer von vier Wochen oder mehr. Foth et al. beschrieben in ihrer Analyse von Okkludern zum Verschluss septaler Defekte einen ähnlichen zeitlichen Ablauf (Foth et al. 2009). Sie detektierten eine vollständige Endothelschicht ab Implantationszeiten von zehn Wochen. Frazier et al. untersuchten immunhistochemisch zwei linksventrikuläre Kreislaufunterstützungssysteme, deren Pumpmembranen mit Polyurethan beschichtet waren. Auf diesem Kunststoffgewebe konnten sie in einem System nach 189 Tagen Endothelzellen durch positive Färbungen für von-Willebrand-Faktor sowie für LDL-Rezeptoren nachweisen (Frazier et al. 1993).

Bezüglich des Ursprungs der Endothelzellen auf Implantaten zeigten Shi et al. in einem Tiermodell mit sechs Hunden, dass endotheliale Vorläuferzellen aus dem Knochenmark in die periphere Zirkulation mobilisiert wurden. Sie bildeten in Gefäßprothesen aus PTFE endotheliale Oberflächen. Die Zellen formten ein einlagiges Pflastersteinrelief und färbten wie in der vorliegenden Arbeit positiv für von-Willebrand-Faktor (Shi Q. et al. 1998). Hu et al. bestätigten die 
Abstammung lumenseitiger Endothelzellen von Vorläuferzellen aus dem Knochenmark des Empfängers (Hu et al. 2003). Auch Rafii et al beschrieben, dass endotheliale Vorläuferzellen im Knochenmark existierten, die in die Blutbahn migrieren, proliferieren und zu reifen Endothelzellen differenzieren könnten. Diese Zellen bildeten eine anti-thrombogene Oberfläche in Gefäßen aus (Rafii et al. 2002). Dem gegenüber kamen mehrere Gruppen zu dem Ergebnis, auf den Oberflächen implantierter Grafts formiere sich keine neue Endothelschicht. Mitchell et al. und Vogt et al. konnten mit Färbungen für von-WillebrandFaktor kein Endothel auf explantierten kryokonservierten Homograft-Klappen nachweisen (Mitchell et al. 1995 und Vogt et al. 1999). Die Implantationszeiten der untersuchten Conduits lagen bei zwei bis vier Monaten (Mitchell et al.) bzw. zwei Wochen bis sieben Jahren (Vogt et al.). Es ist anzumerken, dass in der Veröffentlichung von Vogt et al. keine positiven Kontrollfärbungen erwähnt werden. Daher bleibt unklar, ob zuverlässige Methoden angewandt wurden. Neves et al. fanden an explantierten Homograft-Klappen $(n=7)(\mathrm{Im}-$ plantationszeiten 2 Monate bis 20 Jahre) ebenso keine Endothelzellen (Neves et al. 1995). Dagegen beschrieben sie das Endothel an zum Vergleich untersuchten Klappen aus Herztransplantationen (Implantationszeiten 1 Monat bis 5 Jahre) und einer Autograft-Klappe (Implantationszeit 9 Monate) als teilweise erhalten. Die Untersucher stützen ihre Aussagen auf rein morphologische Auswertungen in HE- und EvG-Färbungen. Ein verlässlicherer Nachweis von Endothel ist jedoch mit immunhistochemischen Färbungen zu führen, wie er in der vorliegenden Arbeit mit Antikörpern gegen von-Willebrand-Faktor erfolgte. Koolbergen et al. untersuchten 40 explantierte Homograft-Klappen (Implantationszeiten 2 Wochen bis 16 Jahre) (Koolbergen et al. 2002a). Sie beschrieben in 30\% der Präparate in der HE-Färbung eine einlagige Schicht an der Conduit-Wand und den Klappensegeln. Zum Nachweis von Endothelzellen verwendeten sie den spezifischen monoklonalen Antikörper PAL-E. Mit diesem konnten sie lediglich in 5\% der Präparate (Implantationszeiten 5 bzw. 59 Monate) eine partielle Endothelialisierung (d.h. multifokal oder zu mehr als 50\% der Oberfläche) nachweisen. Die übrigen Klappen zeigten keine oder nur eine fokale positive Färbung für PAL-E. Dagegen färbte allerdings das Endothel der Kapillaren im umgebenden Gewebe deutlich positiv. Koolbergen et al. beurteilten die Neoendothelialisierung der Homograft-Klappen daher insgesamt als vernachlässigbar. Sie folgerten, Spender-Endothelzellen könnten kaum oder gar nicht regenerieren und Empfänger-Endothelzellen ersetzten die Endothelschicht nicht.

In der vorliegenden Arbeit wurde mit 24 Conduits eine vergleichsweise große Serie untersucht. In den Präparaten ließ sich eine lumenseitige Endothelschicht nachweisen. Dabei dienten als interne Kontrolle die ebenfalls positiv für vonWillebrand-Faktor färbenden Kapillaren der Adventitia. Die untersuchten Conduits waren für eine Dauer von mindestens sechs Monaten implantiert. Aussagen bezüglich einer Endothelialisierung innerhalb des ersten halben Jahres post implantationem konnten wir daher nicht treffen. Die Implantationszeiten der von Mitchell et al. beschriebenen nicht-endothelialisierten Homograftklappen lagen zwischen zwei und vier Monaten. Möglicherweise erfolgt eine Neoendothelialisierung implantierter Homograft-Klappen somit erst nach mehr als vier Monaten. Trotz Widerspruch zu Autoren wie Koolbergen et al. konnten wir 
mit der von uns angewandten validen Methodik zeigen, dass nach primärem Verlust von Spenderendothel in implantierten RVOT-Conduits eine vollständige Neoendothelialisierung der blutzugewandten Oberflächen erfolgt. Sie kann nach sechs Monaten abgeschlossen sein. Die Befunde dieser Arbeit sind vereinbar mit der Theorie der Neoendothelialisierung von Implantaten durch Progenitorzellen des Empfängers wie von Shi, $\mathrm{Hu}$ und Rafii beschrieben ( $\mathrm{Hu}$ et al. 2003, Rafii et al. 2002 und Shi Q et al. 1998).

\subsection{Pseudointima}

In der vorliegenden Arbeit wird das neu gebildete Gewebe, das sich lumenseitig des implantierten Graft-Gewebes befand, als Pseudointima bezeichnet. In der Literatur wird die so benannte Struktur mit einer Vielzahl unterschiedlicher Begriffe beschrieben. Neben pseudointima (Molina et al. 1995 und Zhang und Williams 1992) sind weitere Beispiele neointima (Agarwal et al. 1982, Shebani et al. 2006 und Tamura et al. 2007), neointimal proliferation (Ishizaka et al. 2003), neo-intimal sheath (Jashari et al. 2001), peel formation (Boethig et al. 2005 und Kim et al. 2007), pseudointimal/fibrointimal/intimal peel formation (Schreiber et al. 2006), fibrous peel (Tiete et al. 2004), intimal hyperplasia (Hayabuchi et al. 2007), intimal fibrosis (Pearl et al. 2002), intima thickening/peeling (Breymann et al. 2004), pannus (Berger et al. 1972, Bottio et al. 2003 und Butany et al. 2004) oder fibrous sheathing (Koolbergen et al. 2002a). Unabhängig von der Benennung wird dieses Gewebe in der Literatur als Substrat von Graftstenosierungen angesehen. Da es sich bei der Conduit-Stenose um die Hauptindikation für Explantationen handelt (Cleveland et al. 1992, Homann et al. 2000, Kim et al. 2007, Mitchell et al. 1995, Raanani et al. 2000 und Stark 1998), ist die Neubildung von Gewebe in Conduits auch klinisch von großer Bedeutung.

In allen untersuchten Präparaten befand sich lumenseitig Pseudointima-Gewebe, das innerhalb der einzelnen Conduits sowohl zirkulär als auch im longitudinalen Verlauf unterschiedlich ausgeprägt war. Makroskopisch wies die Pseudointima generell eine glatte, weißliche Oberfläche auf. Feingeweblich setzte sich die Pseudointima unabhängig vom Conduit-Typ aus spindelförmigen Zellen und umgebender faserreicher Extrazellulärmatrix zusammen. Sie zeigte sich entlang der Conduit-Wand und auch an den Klappensegeln. In mehreren Grafts wurden durch die lumenseitige Pseudointima Unregelmäßigkeiten der Graftoberfläche ausgeglichen. Dies förderte möglicherweise einen laminaren Blutfluss. Abschnitte mit verengtem Lumen lagen sowohl auf Klappenhöhe als auch im weiteren Verlauf der Conduits. In der Literatur ist ebenfalls dokumentiert, dass Conduit-Stenosen in unterschiedlichen Lokalisationen auftreten können (Agarwal et al. 1981, Boethig et al. 2005, Brown et al. 2005, Edwards et al. 1983, Ishizaka et al. 2003, Pearl et al. 2002, Sanatani et al. 2001 und Schreiber et al. 2006).

\subsubsection{Muskelzell-Marker}

In der vorliegenden Arbeit zeigten sich die spindelförmigen Zellen in der Pseudointima parallel angeordnet und wiesen in Bezug auf den Conduit eine zir- 
kuläre Ausrichtung auf. Es ist denkbar, dass dies durch den Blutfluss durch den Conduit bedingt war. In der Literatur sind hinsichtlich des PseudointimaAufbaus ebenfalls spindelförmige Zellen beschrieben, die sich parallalel zur Lumenoberfläche angeordnet innerhalb von Kollagenfasern befanden (Bottio et al. 2003 und Meyns et al. 2004). Zur weiteren Charakterisierung der Zellen wurden verschiedene Differenzierungsmarker glatter Muskelzellen eingesetzt. Die wichtigsten kontraktilen Proteine glatter Muskelzellen sind smooth muscle actin (SMA) und smooth muscle myosin (SMMy). Die h-Caldesmon (heavyCaldesmon) Isoform ist an der Regulierung der Kontraktion glatter Muskelzellen beteiligt. Bei Vimentin und Desmin handelt es sich um Filamentproteine und damit Bestandteile des Zytoskeletts. SMA und Vimentin sind regelhaft schon bei gering differenzierten glatten Muskelzellen nachweisbar (Sartore et al. 2001). Dagegen sind h-Caldesmon und Desmin Marker für höher differenzierte Zellen, SMMy nimmt eine Mittelstellung unter den Reifungsmarkern ein (Aikawa et al. 1997, Frid et al. 1997 und Yoshida und Owens 2005).

Die Pseudointima-Zellen der untersuchten Präparate färbten in den immunhistochemischen Färbungen positiv für SMA, Vimentin und meist auch SMMy. Somit besaßen sie Muskelzelleigenschaften. Zusätzlich wiesen sie morphologisch eine deutliche Ähnlichkeit mit Fibroblasten auf. Daher wurden sie in der vorliegenden Arbeit als fibromuskuläre Zellen bezeichnet. Eine positive Färbung für Desmin fand sich schon nach der kürzesten Implantationszeit von einem halben Jahr. Fibromuskuläre Zellen der Pseudointima, die positiv für h-Caldesmon färbten, wurden ab Implantationszeiten von zehn Monaten nachgewiesen. Es kamen also bereits nach sechs Monaten höher differenzierte fibromuskuläre Zellen in der Pseudointima vor. Ein ähnliches Antigenexpressionsmuster wiesen auch Foth et al. in explantierten Okkludern für septale Defekte nach (Foth et al. 2009). Allerdings beobachteten sie höher differenzierte fibromuskuläre Zellen erst ab einer Implantationszeit von mindestens zehn Monaten.

Mehrere Autoren vertraten bezüglich unterschiedlicher Implantattypen die These, dass die Zellen der Pseudointima von im Blut zirkulierenden differenzierungsfähigen Zellen des Empfängers abstammten (Agarwal et al. 1982 und Scott et al. 1994). Nach Daten von Shimizu et al. differenziere sich aus zirkulierenden Knochenmarkszellen möglicherweise auch der Großteil der glatten Muskelzellen der Pseudointima (Shimizu et al. 2001). Shi et al. differenzierten nach Dilatation von orthotopen Schweine-Koronararterien mit immunhistochemischen Färbungen Zellen der verschiedenen Gefäßwandschichten (Shi Y et al. 1996). Ortsständige Fibroblasten der Adventitia waren negativ für SMA und Desmin, glatte Muskelzellen der Media positiv für beide Marker und in die neointima eingewanderte Myofibroblasten positiv für SMA aber negativ für Desmin. In den Präparaten der vorliegenden Arbeit hingegen färbten Zellen der Pseudointima zum Teil positiv für beide genannten Marker. Da sich die Nachbeobachtungszeiten in der Studie von Shi et al. und dieser Arbeit unterschieden und es sich zudem um unterschiedliche Gefäßtypen handelte, können wir keine verlässlichen Angaben zum Verlauf der Differenzierung fibromuskulärer Zellen in Conduits treffen. Es ist aber zu vermuten, dass eine zeitabhängige Reifung der Zellen erfolgt, wie sie beispielsweise für das in humanen Koronararterien nach Angioplastie neu gebildete lumenseitige Gewebe beschrieben wurde (Aikawa et al. 1997). 


\subsubsection{Extrazellulärmatrix-Komponenten}

Die Extrazellulärmatrix der Pseudointima bestand vorwiegend aus dichten, meist gewellten Fasern. Es ließen sich neben elastischen Fasern Kollagenfasern des Subtyps I und noch ausgeprägter des Subtyps III nachweisen. Kollagen I und III sind typische Bestandteile der Extrazellulärmatrix menschlicher Arterienwände (Newby und Zaltsman 2000). Koolbergen et al. beobachteten in Homograftklappen eine Degeneration kollagener und elastischer Fasern im Zusammenhang mit einer Verringerung der Zellzahl ab Implantation (Koolbergen et al. 2002a): Nach Implantationszeiten von einem Jahr sei ein Verlust der Gewebearchitektur feststellbar. Die ursprünglich strukturiert angeordneten Kollagenbündel seien zu einer amorphen Masse degeneriert. Das Volumen an Grundsubstanz sei höher als normal. Koolbergen et al. vermuteten, die degenerativen Veränderungen führten zu einer Verschlechterung der biomechanischen Eigenschaften der Klappen. Dagegen erklärten Vogt et al., Goffin und Mitchell et al., das Kollagennetz von Homograftklappen bleibe größtenteils erhalten und stelle somit die Basis für einen langfristigen Funktionserhalt dar (Goffin 1997, Mitchell et al. 1995 und Vogt et al. 1999). Da Mitchell et al. die Bindegewebszellen der Klappen als avital einstuften, folgerten sie, es komme nicht zu einer Erneuerung der Extrazellulärmatrix. Langfristig kumulierten Schäden des Kollagennetzes somit. Einen guten Erhalt der Kollagenfasern bei schlechtem Erhalt ortsständiger Zellen dokumentierten Bottio et al. für porcine Klappen (Bottio et al. 2003).

\subsubsection{Entstehungsmechanismen der Pseudointima}

Außer den beschriebenen bindegewebig organisierten Bereichen wies die Pseudointima vielfach Regionen mit aufgelockerter Struktur auf. Hier bestand sie entweder hauptsächlich aus Septen (zum Teil als Fibrinfäden identifizierbar) oder aus thrombotischem Gewebe. Dieses war gekennzeichnet durch zusätzliches amorphes Material sowie Blutzellen zwischen den Septen. Die inflammatorischen Zellen und Erythrozyten fanden sich stellenweise zahlreich. Die in diesem Kapitel beschriebenen thrombotischen Pseudointima-Bereiche müssen von wandständigen Thromben im eigentlichen Sinne unterschieden werden. Auf diese wird unter 4.6 eingegangen.

Die aufgelockerten Pseudointima-Bereiche können als Zwischenstufe in der Entwicklung zum bindegewebigen Aufbau mit dicht gelagerten Fasern und fibromuskulären Zellen aufgefasst werden. Einzelne Areale unterschiedlicher histologischer Struktur zeigten sich auch in Übergangsformen nebeneinander innerhalb der Conduits. In der Literatur ist mehrfach dokumentiert, dass in kardiovaskulären Implantaten durch Organisation von initial thrombotischem Material eine bindegewebige Pseudointima aus Extrazellulärmatrix mit eingebetteten fibromuskulären Zellen gebildet wird. Derartige Umwandlungsprozesse beschrieben beispielsweise Sigler und Jux in Okkludern für Vorhofseptumdefekte sowie Valente et al. in Hancock-Conduits (Sigler und Jux 2007 und Valente et al. 1992).

Generell fanden sich die septierten und thrombotischen Pseudointima-Areale insbesondere dort, wo verlangsamte und turbulente Blutfluss-Bedingungen vorherrschten: Während entlang der zirkulären Conduit-Wand die bindegewebige 
Organisation mit parallel angeordneten fibromuskulären Zellen deutlich überwog, bestand die Pseudointima an Klappensegeln, in Graftfalten und Klappensinus vermehrt aus Septen mit oder ohne zwischengelagerter amorpher Substanz. Diese Befunde deuteten auf eine Flussabhängigkeit der PseudointimaStruktur hin. Auch die histologischen Befunde im von uns untersuchten klappenlosen PTFE-Graft stützten die These bezüglich rheologischer Einflussfaktoren auf die Pseudointima-Struktur. Hier war die Pseudointima lumenseitig völlig gleichmäßig ausgebildet und durchgehend bindegewebig (ohne Septen) organisiert. Die Ursache hierfür dürfte außer in der langen Implantationszeit zudem in den wenig turbulenten und eher laminaren Flussbedingungen im Vergleich zu den klappentragenden Conduits gelegen haben.

Shebani et al. dokumentierten fibrinreiches thrombotisches Material mit Entzündungszellen an den Klappensegeln von Rindervenengrafts (Shebani et al. 2006). In einem nach neun Jahren aus der Pulmonalisposition explantierten Pulmonalis-Homograft wiesen Butany et al. ebenfalls ausgeprägt neu gebildete Pseudointima nach, die sie als pannus und intima bezeichneten (Butany et al. 2004). Sie befand sich entlang des Conduit-Gewebes und an beiden Seiten der Klappensegel. Es zeigten sich fokale Verkalkungen, die sich in das Lumen hineinwölbten und in deren unmittelbarer Nähe das Pseudointima-Gewebe besonders deutlich ausgeprägt war. Die Untersucher vermuteten, durch die Vorwölbung ausgelöste Turbulenzen des Blutflusses hätten zu einer progressiven zellulären intima-Proliferation geführt. Diese These deckt sich mit den Ergebnissen der vorliegenden Arbeit hinsichtlich Entstehungsmechanismen der Pseudointima.

Houel et al. erhoben ähnliche Befunde an den zu- und abführenden Conduits von fünf explantierten linksventrikulären Kreislaufunterstützungssystemen (Implantationszeiten zwischen 61 und 226 Tagen) (Houel et al. 1999). In den Inflow-Conduits wiesen sie unterschiedlich ausgeprägt PseudointimaGewebe nach. Es bestand aus EZM-Komponenten und Zellen. Immunhistochemische Färbungen identifizierten Kollagenfasern der Typen I und III in lockerer Anordnung, Fibrinansammlungen sowie Chondroitinsulfat und Dermatansulfat als amorphe Bestandteile der Extrazellulärmatrix. Es fanden sich viele SMA-positive Zellen in ungeordnetem Muster (bis 150 Tage). Nach längerer Implantationszeit (202 Tage) waren sie deutlicher zirkulär ausgerichtet. Lumenseitig waren wenige Endothelzellen nachweisbar. Zwischen der Pseudointima und dem Polyester-Gewebe beschrieben Houel et al. Blutkoagel, die vor allem aus Fibrin bestanden. Es ist zu vermuten, dass sie der fibrinreichen thrombotischen Pseudointima entsprachen, die zum Beispiel von Agarwal et al. (s. u.) sowie in der vorliegenden Arbeit dokumentiert wurde. Die Pseudointima des Outflow-Conduits unterschied sich im Vergleich zu der des Inflow-Conduits deutlich. Sie war dünner, adhärent an der Graftwand und zeigte eine stärkere zirkuläre Anordnung der SMA-positiven Zellen. Houel et al. betonten die Bedeutung lokaler hämodynamischer Gegebenheiten für die Entstehung der Pseudointima. Der im Inflow-Conduit niedrigere Druck sowie die langsamere Flussgeschwindigkeit und damit die schwächeren Scherkräfte auf die Oberfläche seien verantwortlich gewesen für die geringere Adhärenz der Pseudointima am Polyestergewebe und die unstrukturierte Anordnung der fibromuskulären Zellen. Die Fibrinablagerungen zwischen bindegewebig orga- 
nisierter Pseudointima und Inflow-Conduit führten sie ebenfalls auf die lokalen rheologischen Bedingungen zurück.

Agarwal et al. untersuchten 14 schweineklappentragende Conduits nach Explantation aus dem rechtsventrikulären Ausflusstrakt (Implantationszeiten zwischen 1 Tag und 5 Jahren) (Agarwal et al. 1982). In allen Präparaten zeigte sich lumenseitig Pseudointima-Gewebe (neointima, peels), das die Lumina nicht obstruierte. Agarwal wies nach, dass die Bildung der Pseudointima am Tag 1 post implantationem als dünne Lage thrombotischen Gewebes aus Plättchen und Fibrin begann. Danach kam es zu einem Einschluss von Erythrozyten und einigen Leukozyten. Nach Implantationszeiten von zwei bis drei Wochen war die dünne thrombotische Schicht durch fibroblastische Migration und Proliferation von Fibroblasten von der proximalen und distalen Conduit-Anastomose organisiert. Die dichte fibröse lumenseitige Oberfläche zeigte nach einem Monat keine Veränderungen mehr. In der Zone zwischen bindegewebiger Pseudointima und Conduit befand sich dagegen weiterhin thrombotisches Material, wie es sich auch in Präparaten der vorliegenden Arbeit darstellte (Vgl. Abb. 3.56B). Agarwal et al. nahmen an, die fortschreitende Verdickung der Pseudointima erfolge ab Implantationszeiten von einem Monat primär in dieser Zwischenzone.

In der vorliegenden Arbeit konnten keine genauen zeitlichen Abläufe der Pseudointima-Entwicklung bestimmt werden, weil die kürzeste Implantationszeit der Condiuts sechs Monate betrug. Auf Grund zahlreicher Übereinstimmungen der histologischen Befunde mit Daten der Literatur ist aber davon auszugehen, dass grundsätzlich die Ablagerung thrombotischen Gewebes der Ausbildung einer bindegewebigen Pseudointima vorausgeht. Unsere Ergebnisse lassen vermuten, dass diese Prozesse kontinuierlich - sowohl kurz nach Implantation als auch noch nach Jahrzehnten - ablaufen können. Des Weiteren deuteten Befunde dieser Arbeit im Gegensatz zu Agarwals Ergebnissen darauf hin, dass eine Gewebeneubildung dauerhaft auch an der lumenseitigen Conduit-Oberfläche erfolgen kann. Übereinstimmend mit Agarwal gehen wir davon aus, dass bestimmte rheologische Verhältnisse förderlich auf die Entstehung von PseudointimaGewebe wirken. Lin et al. beschrieben, dass durch eine Oberflächenbeschichtung von PTFE-Grafts mit Heparin die Pseudointima-Bildung reduziert werde (Lin et al. 2004). Dieses Ergebnis stützt den Befund, dass diese Gewebebildung über eine Ablagerung thrombotischen Materials verläuft. Trotzdem hat sich eine Heparinbeschichtung von RVOT-Conduits klinisch nicht durchgesetzt.

Bei der Analyse der vorliegenden Präparate fiel auf, dass die Hancock-Conduits eine im Vergleich besonders ausgeprägte Pseudointima mit ausgedehnten septierten und thrombotischen Bereichen aufwiesen. So waren mehrfach die Klappensinus mit aufgelockertem Pseudointima-Gewebe gefüllt und in drei Präparaten Klappensegel von Pseudointima überdeckt. Starke und rasche Gewebeneubildung in porcinen Implantaten sowie eine frühe Conduit-Stenosierung mit teilweise vollständig umschlossenen Klappensegeln und konsekutiver Immobilität sind auch in der Literatur mehrfach dokumentiert worden (Boethig et al. 2005, Butany et al. 1999, Ishizaka et al. 2003, Kim et al. 2007, Pearl et al. 2002 und Schreiber et al. 2006). Diese Pseudointima-Bildung könnte auf eine Fremdkörper- bzw. Entzündungsreaktion des Empfängerorganismus gegen das Xenograft-Gewebe zurückzuführen sein (vergleiche Kapitel 4.5.2). 
Als Therapieoption bei überschießender Gewebeneubildung, die zu einer Graftstenosierung führt, ist in der Literatur für alle drei untersuchten ConduitTypen eine interventionelle Aufdehnung (Angioplastie) mit oder ohne Stentimplantation beschrieben worden (Perron et al. 1999 und Stanfill et al. 2008). In den Präparaten der vorliegenden Arbeit ließ sich zwischen dilatierten und nicht interventionell behandelten Conduits insgesamt kein signifikanter Unterschied hinsichtlich Quantität oder Qualität der Gewebereaktion feststellen. Ergebnisse der Literatur verdeutlichen jedoch, dass eine übermäßige Dilatation von Gefäßen die Pseudointima-Bildung verstärken und so zu erneuter Stenosierung führen könne (Aikawa et al. 1997 und Farb et al. 2002). Auch in Gefäßen an orthotoper Position kommt es unter bestimmten Bedingungen zu einer lumenseitigen Gewebezunahme, die Ähnlichkeiten zur PseudointimaBildung in Conduits aufweist. Als generelle Hauptstimuli sind in der Literatur Gefäßwand-Verletzungen, Entzündung und gesteigerte Scherkräfte auf die Wand beschrieben (Newby und Zaltsman 2000). Shi et al. wiesen in orthotopen Koronararterien von Schweinen nach, dass eine lumenseitige Gewebeneubildung nach Gefäßwandschädigung über thrombotische Zwischenstufen verläuft (Shi Y et al. 1996).

\subsubsection{Chondroide Metaplasie}

Eine chondroide Metaplasie (englisch: cartilaginous metaplasia) ist definiert als Anwesenheit von Chondrozyten in Hohlräumen innerhalb einer kollagenen, mukopolysaccharidreichen Matrix (Qiao et al. 1995). In der Pseudointima weniger Präparate waren in der Färbung nach Richardson Zellen erkennbar, die eine von den bereits beschriebenen, fibromuskulären Zellen abweichende Morphologie aufwiesen: Sie waren wabig in der amorphen Matrix angeordnet und enthielten große runde Zellkerne. Damit entsprachen diese PseudointimaAreale einer chondroiden Metaplasie, wie sie auch in der Literatur beschrieben ist. Mathieu et al. werteten Aortenallografts aus Ratten histologisch aus (Implantationszeiten 30 bzw. 180 Tage) (Mathieu et al. 2003). Sie beschrieben in der Pseudointima innerhalb einer faserreichen Extrazellulärmatrix Chondrozyten, die negativ für SMA und positiv für S100-Protein - einen Marker für Chondrozyten - färbten. Mathieu et al. stellten die These auf, dass sich in Aortenallografts Gewebe aus überwiegend SMA-positiven Zellen über eine Zwischenstufe zu einer chondroiden Metaplasie entwickle. Diese Transformation sei mit einer verstärkten Expression des transforming growth factor $\beta 1$ (TGF B1) assoziiert. Durch endochondrale Ossifikation bilde sich schließlich eine ausgeprägte Kalzifikation der Graftwand aus. In den Verkalkungsarealen lasse sich durch positive Färbung für alkalische Phosphatase osteoblastische Aktivität nachweisen.

Schulick et al. untersuchten den Einfluss einer lokal erhöhten Expression von TGF 31 in unverletzten orthotopen Rattenarterien (Schulick et al. 1998). Sie beobachteten die Bildung einer neointima. In dieser neointima und noch stärker ausgeprägt in der Media der Arterien detektierten sie eine chondroide Metaplasie. Die Chondrozyten seien durch Umwandlung glatter Muskelzellen der Gefäßwand (VSMCs) entstanden und zeichneten sich folglich durch eine große Plastizität aus. Die Chondrozyten färbten positiv für S100-Protein und 
negativ für SMA und machten 10 bis 25\% aller Zellen in Media und neointima aus. Sie wiesen eine runde Form und große Zellkerne auf und befanden sich in Hohlräumen, die von einer lockeren Extrazellulärmatrix aus Kollagenfasern und reichlich Proteoglykanen umgeben waren. Unter Entzug von TGF B1 bildeten sich die chondroide Metaplasie und das neointima-Gewebe durch massive Apoptose zurück. Schulick et al. stellten zu keinem Studienzeitpunkt eine Mineralisation in den Arterien fest. Es sei also nicht zu einer Kalzifikation gekommen, obwohl die Autoren betonten, dass angesichts der Plastizität der VSMCs auch dies unter dem Einfluss entsprechender Wachstumsfaktoren denkbar sei.

Einflussfaktoren und Auswirkungen einer chondroiden Metaplasie untersuchten Qiao et al. in der Aorta von Mäusen (Qiao et al. 1995). Sie vermuteten eine genetische Ursache für ihr Auftreten. Außerdem könne mechanischer Stress zu chondroider Metaplasie führen, weshalb sie hauptsächlich im Klappenapparat lokalisiert sei. Qiao et al. wiedersprachen aber einer Abhängigkeit von Immunreaktionen. Eine chondroide Metaplasie sei eine Zwischenstufe zu arterieller Kalzifikation und Atherosklerose. In den von chondroider Metaplasie betroffenen Präparaten der vorliegenden Arbeit zeigte sich zusätzlich eine ausgeprägte Verkalkung der Pseudointima. Somit könnten die Areale chondroider Metaplasie - wie in der Literatur beschrieben - einer Vorstufe von Kalzifikation entsprochen haben.

\subsection{Neovaskularisierung und Lakunen}

\subsubsection{Kleine Gefäße und Kapillaren}

Die Vasa vasorum orthotoper menschlicher Gefäße befinden sich im Bindegewebe der Adventitia und in äußeren Anteilen der Media; die Intima enthält hingegen keine Blutgefäße. Im Pseudointima-Gewebe der analysierten HancockConduits und Homografts sowie zwei der drei Rindervenengrafts fanden sich jedoch Kapillaren und kleine Gefäße. Diese wiesen lumenseitig eine Schicht vonWillebrand-Faktor-positiver Endothelzellen auf, die von unterschiedlich vielen Lagen fibromuskulärer Zellen umgeben waren. Die Zellen waren zirkulär angeordnet und färbten meist positiv für SMA und Vimentin. Zum Teil exprimierten sie darüber hinaus Antigene wie SMMy, h-Caldesmon und Desmin, die auf eine fortgeschrittene Differenzierung hinwiesen. Damit unterschieden sich die Zellen der Gefäßwände in ihrem Reifungsmuster in mehreren Präparaten von den weniger differenzierten fibromuskulären Zellen in der umgebenden Pseudointima. Offenbar erfolgte in den fibromuskulären Zellen der Gefäßwände also eine schnellere Reifung.

In den Hancock-Conduits bestand mehrfach eine Häufung der Kapillaren in der Nähe des Graftmaterials. Teilweise fanden sich perivaskulär viele Entzündungszellen. Eine mögliche Erklärung für diese Beobachtung ist die Einwanderung inflammatorischer Zellen über die neu gebildeten Gefäße.

In der Literatur gibt es keine Arbeiten, die sich mit der Neoangiogenese in Conduits im rechtsventrikulären Ausflusstrakt befassen. Mehrere Autoren erwähnen aber Kapillaren in ihren histopathologischen Auswertungen explantierter Grafts als Teilaspekt. In verschiedenen Studienanordnungen wurde ein Einwachsen 
von Gefäßen in die Wand von Kunststoffgrafts beobachtet (Zhang und Williams 1992 und Müller und Dasbach 1994). Ando et al. vermuteten ein Einsprossen durch die Stichkanäle ausgehend von der Adventitia (Ando et al. 2000). Hu et al. untersuchten arterielle Allografts in Mäusen (Hu et al. 2003). Sie stellten fest, dass die Bildung neuer Kapillaren in den Grafts von Vorläuferzellen ausging, die aus dem Knochenmark des Empfängers stammten. Die neugebildeten Gefäße zeigten sich in Pseudointima, Media und Adventitia. Diese Neoangiogenese sei der Bildung von Pseudointima-Gewebe vorausgegangen. Weiterhin ist eine Kapillarisierung der Pseudointima in Okkludern für den interventionellen Verschluss von Vorhofseptumdefekten beschrieben worden (Foth et al. 2009). In ortsständigen Rattenarterien wiesen Diaz-Flores et al. neu gebildete Kapillaren nach, die ebenfalls von der Mikrozirkulation der Adventitia ausgehend in Media und Intima zur Versorgung dieser GefäßwandSchichten hineinwuchsen (Dìaz-Flores et al. 1990). Dies legt die Vermutung nahe, dass es sich bei der in Conduits beobachteten Neovaskularisierung zumindest zum Teil um physiologische Mechanismen des Empfängerorganismus handelte.

Native Herzklappen sind im gesunden Zustand weitgehend frei von Gefäßen. Es finden sich allenfalls vereinzelte Kapillaren an der Klappenbasis. Einige der untersuchten Grafts enthielten jedoch im Pseudointima-Gewebe der Klappensegel Kapillaren mit intraluminal nachweisbaren Erythrozyten. Tamura et al. untersuchten native Mitralklappensegel von Hunden, an denen Inzisionen gesetzt und wieder vernäht worden waren (Tamura et al. 2007). Anschließend fand sich thrombotische Pseudointima mit Kapillaren an den Klappen. Einige dieser Gefäße gingen vom Klappenring aus. Hieran wird deutlich, dass auch an orthotopen Klappen unter bestimmten Bedingungen eine Neovaskularisierung erfolgen kann. Diese steht offenbar im Zusammenhang mit Heilungsprozessen. Dong et al. und Farb et al. stellten fest, Angiogenese sei im Rahmen reaktiver und pathologischer Vorgänge mit Entzündung assoziiert (Dong et al. 2009 und Farb et al. 2002). Dabei seien Makrophagen in der Lage, sowohl fördernd als auch hemmend auf die Angiogenese zu wirken. Als Konsequenz hieraus war möglicherweise die in unseren Präparaten beobachtete räumliche Assoziation von Entzündungszellen mit kleinen Gefäßen interpretierbar. Auch im Zusammenhang mit der ausgeprägten Fremdkörperreaktion auf das Graftgewebe der Hancock-Conduits ließ sich somit die Häufung von Kapillaren in unmittelbarer Nähe des PTFE-Materials erklären. Moulton sowie Jeziorska und Woolley betonten die Bedeutung der Angiogenese im Zusammenhang mit der Entstehung atherosklerotischer Plaques (Moulton 2001 und Jeziorska und Woolley 1999). Auch hierbei sprösse ein Netz von Kapillaren von den Vasa vasorum der Adventitia ausgehend durch die Gefäßwand in die Intimaläsionen ein. So könnte die Neubildung der Gefäße in den von uns untersuchten Grafts zu deren Mineralisierung beigetragen und somit letztlich ein funktionelles Versagen beschleunigt haben.

Als Perizyten werden in der Literatur perivaskuläre Zellen bezeichnet, die in präkapillären Arteriolen, Kapillaren und postkapillären Venolen zu finden sind (Collett und Canfield 2005). Sie seien länglich mit vielen Fortsätzen und lägen den Endothelzellen benachbart. Perizyten hätten Ähnlichkeit mit Myofibroblasten und färbten positiv für SMA (Doherty et al. 1998 und Farrington- 
Rock et al. 2004). Als kontraktile Zellen seien sie beteiligt an der Regulation von Gefäßkaliber und -permeabilität sowie der mechanischen Stabilisierung der Gefäßwand (Collett und Canfield 2005). In Studien wurde ein ausgesprochen breites Differenzierungspotential von Perizyten beschrieben (Doherty et al. 1998 und Farrington-Rock et al. 2004). So könnten sie zu Knorpel-, Knochenund Bindegewebs-Zellen differenzieren. Sie trügen bei zu Wachstum, Heilungsprozessen sowie pathologischen Vorgängen wie vaskulärer Kalzifizierung und chondroider Metaplasie in Gefäßen. Diese in orthotopen Gefäßen beschriebenen Perizyten weisen deutliche morphologische Ähnlichkeit auf zu Zellen der Wand kleiner Gefäße in explantierten Grafts, wie sie auch in der vorliegenden Arbeit beschrieben sind. Möglicherweise handelt es sich somit um den gleichen Zelltyp mit folglich auch ähnlichen funktionellen Eigenschaften. Daher ist denkbar, dass den Zellen der Wand neugebildeter kleiner Gefäße eine wichtige Rolle zukommt bei der Bildung der Pseudointima und beispielsweise auch bei Mineralisierungsprozessen. Folgerichtig könnte es sich bei den Zellen der Gefäßwände und den fibromuskulären Zellen in der umgebenden Pseudointima trotz gewisser Ähnlichkeiten um unterschiedliche Zelltypen gehandelt haben. Dies wäre eine Erklärung für das abweichende Reifungsmuster der Muskelzellen im Sinne einer schnelleren Differenzierung der Gefäßwand-Zellen gegenüber den übrigen fibromuskulären Zellen der Pseudointima.

\subsubsection{Lakunen}

Als Lakunen wurden in der vorliegenden Arbeit Hohlräume mit EndothelzellAuskleidung bezeichnet, denen jedoch die für Gefäße typischen Lagen muskulärer Zellen im Sinne eines mehrschichtigen Gefäßwand-Aufbaus fehlten. Sie fanden sich in der Pseudointima der meisten Homografts und einiger HanockConduits und wiesen teilweise deutlich größere Lumina als die Kapillaren sowie ausgeprägte Verzweigungen auf. Auch nahe der Metallstreben in einem der gestenteten Homografts zeigten sich viele Lakunen. Es konnte jedoch kein generelles Lokalisationsmuster festgestellt werden. Ebenso wenig bestand eine Korrelation zwischen Anzahl an Lakunen und Implantationszeit der Conduits. In der verfügbaren Literatur gibt es kaum Angaben über Lakunen. Kreischer beschrieb in ihrer histomorphologischen Arbeit Lakunen in dem neu gebildeten Gewebe in Vorhofseptumdefekt-Okkludern. Sie stellte die These auf, eine verstärkte Lakunenbildung könne durch einen fortdauernden Blutstrom durch ein Implantat bedingt sein (Kreischer 2008). Mit den in der vorliegenden Arbeit verwendeten Methoden war letztlich keine völlig klare Abgrenzung zwischen Kapillaren und Lakunen sowie keinesfalls eine sichere Aussage über deren Entstehungsmechanismen möglich. Es ist denkbar, dass sich Lakunen aus den oben beschriebenen bindegewebigen Septen bilden. Möglicherweise handelt es sich um eine Entwicklungsstufe von Kapillaren. So könnten kleine Gefäße zu Lakunen dedifferenzieren oder aber es könnte sich über eine Differenzierung von Zellen um die Endothelzell-Lage der Lakunen herum eine Gefäßwand formieren. 


\subsection{Entzündungsreaktion}

Einer der Schwerpunkte der vorliegenden Arbeit war die Auswertung inflammatorischer Reaktionen in den Conduits. Anderson et al. fassten die Abläufe von Entzündungsreaktionen und Wundheilungsprozessen nach Implantation von körperfremdem Material ausführlich zusammen (Anderson und Miller 1984 und Anderson et al. 2008). Anfangs komme es abhängig vom eingebrachten Material zu einer Anlagerung von Proteinen auf der Graftoberfläche und der Bildung einer transienten, thrombotischen Matrix auf Basis von Blutbestandteilen wie Fibrin. Die folgende akute Entzündungsreaktion sei von neutrophilen Granulozyten dominiert. Sie gehe nach etwa einer Woche in die circa zweiwöchige chronische Entzündungsphase über, die durch mononukleäre Zellen wie Monozyten, Lymphozyten und Plasmazellen am Implantationsort geprägt sei. Schließlich folge über Einwanderung von Makrophagen und Fibroblasten sowie Neovaskularisierung die Bildung von Granulationsgewebe als Vorstufe einer fibrösen Kapsel (fibrous capsule). Vom Implantat abgegrenzt sei das Granulationsgewebe durch die zellulären Komponenten der Fremdkörperreaktion, einer Lage von Monozyten, Makrophagen und Fremdkörperriesenzellen direkt an der Oberfläche des Devices. Durch Chemokine geleitet migrierten im Rahmen der Fremdkörperreaktion Monozyten und von diesen abstammende Makrophagen zum Implantat und adhärierten an die dort angelagerten Proteine. Schließlich fusionierten die Makrophagen zu Fremdkörperriesenzellen. Diese Fusion sei abhängig vom Implantatmaterial und den adhärierten Proteinen sowie entsprechenden fusionsinduzierenden Stimuli. Die Fremdkörperreaktion persistiere während der gesamten Implantationszeit des Devices und beeinflusse dessen Biokompatibilität. Die Reaktion könne so zu Degeneration und eventuell Versagen von Grafts führen. Makrophagen würden unter anderem durch Lymphozyten aktiviert und steuerten ihrerseits Leukozyten wie Neutrophile und Lymphozyten sowie Fibroblasten (Bonfield et al. 1992). Durch diesen Feedback-Mechanismus mit gegenseitiger Verstärkung zwischen Makrophagen und Lymphozyten wirkten die Makrophagen und Fremdkörperriesenzellen auch ein auf die Bildung des fibrösen Gewebes, das unter Umständen die Implantatfunktion beeinträchtigen könne.

\subsubsection{Verwendente Antikörper gegen Oberflächen-Antigene von Ent- zündungszellen}

In der vorliegenden Arbeit erfolgten neben der rein histomorphologischen Untersuchung der Präparate immunhistochemische Färbungen zur genaueren Differenzierung der inflammatorischen Zellen. T-Lymphozyten sind zelluläre Komponenten der erworbenen Immunität. Der Antikörper gegen CD3 zum Nachweis von T-Zellen wurde durchgehend angewandt. Dabei ließen sich T-Zellen in nahezu allen Präparaten und allen Graftschichten identifizieren. Die Färbungen für CD4- und CD8-Antigene zur Unterscheidung in T-Helfer- und TKillerzellen wurden dagegen nur bei einigen Conduits durchgeführt. Die entsprechenden Präparate waren aus technischen Gründen lediglich eingeschränkt beurteilbar. 
Der anfangs ausschließlich verwandte Antikörper gegen CD79 zur Identifikation von B-Lymphozyten färbte unspezifisch auch glatte Muskelzellen an. Mit ihm ließen sich insgesamt nur wenige B-Zellen nachweisen. An einigen Schliffen wurden zusätzlich Färbungen mit dem Antikörper gegen CD79alpha durchgeführt. So war eine bessere Identifizierung von B-Lymphozyten möglich. Daher muss davon ausgegangen werden, dass die Färbungen mit dem ursprünglich gegen CD79 verwendeten Antikörper lediglich über eine begrenzte Aussagekraft verfügten. Plasmazellen sind als Produzenten von Immunglobulinen Träger der humoralen, erworbenen Immunität. Die Färbung für CD138 zum Nachweis von Plasmazellen erfolgte an einer kleinen Zahl von Schliffen. Dabei wurden insgesamt nur in wenigen Präparaten Plasmazellen angefärbt und dort zudem jeweils in geringer Zahl. Letztlich ließ sich nicht sicher bewerten, ob dieses Ergebnis auf eine tatsächlich begrenzte Zahl von Plasmazellen zurückzuführen war oder ob es durch technische Limitationen der Färbemethode bedingt war.

CD68-positive Makrophagen als Teil des angeborenen Immunsystems ließen sich in ähnlich vielen Conduits und in vergleichbar hoher Zahl wie T-Lymphozyten anfärben. Die Makrophagen lagen sowohl als einzelne Histiozyten als auch in Form mehrkerniger Riesenzellen (Fremdkörperriesenzellen) vor.

\subsubsection{Ergebnisse der immunhistochemischen Charakterisierung der Entzündungsreaktion}

In den untersuchten Präparaten ließ sich grundsätzlich und unabhängig vom Grafttyp eine entzündliche Reaktion auf synthetisches Fremdgewebe wie PTFE und Fäden von Anastomosennähten erkennen. Insbesondere zeigten sich CD68-positive Makrophagen in unmittelbarer Nähe dieser Materialien und waren hier oft zu mehrkernigen Fremdkörperriesenzellen fusioniert (Vgl. Abb. 3.21 und 3.66). Die Fremdkörperreaktion auf implantiertes Fremdgewebe mit Ansammlung von Makrophagen und Formation von Fremdkörperriesenzellen an der Oberfläche von Implantaten wurde auch in der Literatur mehrfach beschrieben (Anderson et al. 2008, Foth et al. 2009, Meyns et al. 2004, Sigler et al. 2005 sowie Sigler und Jux 2007).

Anhand der drei gestenteten Homografts sowie der Hancock-Conduits mit umgebendem Metallring zeigte sich, dass auf Metallanteile in den Präparaten keine auffällige Reaktion des umgebenden Gewebes erfolgte: Es waren weder ein verstärktes entzündliches Geschehen noch eine überschießende PseudointimaBildung in der Nähe von Metall zu erkennen. Ebenso wies das Metall selbst keine Veränderungen wie beispielsweise Korrosion auf.

In der Mehrzahl der Präparate zeigten sich die inflammatorischen Zellen unabhängig vom Grafttyp gehäuft in direkter Nähe von Kapillaren und kleinen Gefäßen. Insbesondere in der Pseudointima und der Adventitia ließ sich die perivaskuläre Lokalisation beobachten. Sie galt für alle in dieser Arbeit unterschiedenen Typen von Entzündungszellen und ist auch in der Literatur beschrieben (Jeziorska und Woolley 1999). Diese Beobachtung legte die Vermutung nahe, dass die inflammatorischen Zellen über neu gebildete Blutgefäße in die Conduits einwanderten. 


\section{Entzündungsreaktion in den Homografts}

Die stärkste Entzündungsreaktion innerhalb der untersuchten Homografts zeigte sich durchgehend in der Adventitia. Auch in der Pseudointima war die inflammatorische Reaktion vielfach deutlich zu erkennen, in der Media dagegen nur schwach. Immunhistochemisch ließen sich in der Pseudointima und der Adventitia aller Homografts T-Lymphozyten und Makrophagen nachweisen. Diese Zelltypen fanden sich auch in einigen Klappensegeln, hier jedoch in geringerer Zahl als in der Conduit-Wand.

Bezüglich histologischer Untersuchungen explantierter Homografts liegen verschiedene Studien vor, in denen eine Entzündung in den Conduits dokumentiert ist. In der Mehrzahl der Veröffentlichungen wird eine vorwiegend zellulär getragene Immunreaktion beschrieben. Goffin zeigte, dass eine inflammatorische Reaktion auf implantierte Homografts bereits früh (innerhalb weniger Stunden nach Implantation) auftrat (Goffin 1997). Angesichts von Implantationszeiten von mindestens sechs Monaten in der vorliegenden Arbeit war dies eine wichtige Ergänzung unserer Befunde. Allerdings ging Goffin von einer zeitlichen Limitierung der Entzündung auf etwa zwei Wochen aus. Diese These konnte von uns nicht bestätigt werden: In den Präparaten mit ihren vergleichsweise langen Implantationszeiten von bis zu neunzehneinhalb Jahren ließ sich eine persistierende chronische Immunreaktion erkennen, die vor allem durch Makrophagen und T-Lymphozyten getragen war.

Koolbergen et al. wiesen in explantierten Homografts (Implantationszeiten 2 Wochen bis 16 Jahre) immunhistochemisch Makrophagen und im Verlauf zusätzlich T-Lymphozyten nach (Koolbergen et al. 2002a). Bei Implantationszeiten unter einem Jahr seien die Entzündungszellen überwiegend diffus verteilt in der Pseudointima der Klappen lokalisiert gewesen. Nach mehr als einem Jahr fanden sie sich in kleinen Gruppen vor allem in der Conduit-Adventitia. Koolbergen et al. kamen zu dem Schluss, es läge zwar eine - insgesamt geringgradige - Entzündungsreaktion in den Allografts vor. Sie sahen jedoch keinen Hinweis auf eine immunvermittelte Abstoßung der Implantate. Mitchell et al. identifizierten in explantierten kryokonservierten Homograft-Klappen (Implantationszeiten Stunden bis 9 Jahre) immunhistochemisch ebenfalls geringe Infiltrate aus T-Lymphozyten und Makrophagen (Mitchell et al. 1998). Auch sie gaben an, es bestehe kein Zusamenhang zwischen der Immunantwort und dem Ausmaß der beobachteten degenerativen Veränderungen oder der Geschwindigkeit eines Klappenversagens.

Von den meisten Autoren wird im Gegensatz zu Koolbergens und Mitchells Ergebnissen die These vertreten, Homografts unterlägen einer immunologisch vermittelten Zerstörung, aus der valvuläres Versagen und Conduit-Stenosierungen resultieren könnten. Neves et al. beschrieben, dass eine T-Zell-getragene Immunreaktion zu einem Zell- und Strukturverlust in Allograftklappen führe (Neves et al. 1995). Dieser sei durch eine immunsuppressive Medikation zu verhindern. Legare et al. zeigten im Rattenmodell, dass implantierte Aortengraftklappen innerhalb der ersten Woche von CD3- und CD8-positiven T-Lymphozyten inflitriert wurden (Legare et al. 2000). Nach vier Wochen Implantationszeit war die Hälfte der untersuchten Allograftklappen zerstört und wies eine ausgeprägte Pseudointima-Schicht an den Rudimenten auf. Im Gegensatz hierzu 
blieb die histologische Struktur syngener Klappen erhalten und diese wurden nur von wenigen Immunzellen infiltriert. Allografts in T-Zell-defizienten Ratten wiesen keine strukturellen Veränderungen auf. Legare et al. folgerten, dass es nach Implantation von Allograftklappen zu einer durch T-Lymphozyten vermittelten Abstoßung komme, die über - insbesondere durch zytotoxische T-Zellen mediierte - Apoptose ablaufe. Die Abstoßungsreaktion führe letztlich zum strukturellen Versagen der Klappen. Die Daten von Legare et al. zeigten ein zahlenmäßiges Überwiegen von CD8-positiven gegenüber CD3-positiven Zellen. In der vorliegenden Arbeit waren die zytotoxischen T-Lymphozyten dagegen in deutlich geringerer Zahl anzufärben. Dieses Ergebnis ist insofern besser nachvollziehbar, als dass es sich bei CD8-positiven Zellen um eine Subgruppe der T-Lymphozyten handelt, die allesamt positiv für CD3 färben müssten. Dennoch ist der Nachweis zytotoxischer T-Lymphozyten ein wichtiger Hinweis bezüglich möglicher Ursachen eines Klappenversagens in Allografts.

Auch Rajani et al. stellten in explantierten kryokonservierten Homografts entzündliche Infiltrate in den Klappen fest (Rajani et al. 1998). Die Grafts waren den Patienten im Säuglingsalter implantiert worden und versagten innerhalb von maximal acht Monaten. Immunhistochemisch ließen sich T-Lymphozyten, B-Lymphozyten und Makrophagen nachweisen. Rajani et al. folgerten ebenfalls, eine zelluläre Abstoßung führe zum Versagen von HomograftKlappen. Wie in der vorliegenden Arbeit dokumentierten Rajani et al. ein Überwiegen von T- gegenüber B-Lymphozyten. Ebenso waren alle drei ConduitWandschichten infiltriert, in den Klappen zeigten sich hingegen nur einzelne inflammatorische Zellen. Vogt et al. wiesen in Homograft-Klappen aus dem rechtsventrikulären Ausflusstrakt von Kindern (Implantationszeiten 2 Wochen bis 7 Jahre) immunhistochemisch eine T-Zell-dominierte Entzündungsreaktion in der Graftwand nach (Vogt et al. 1999). Außerdem zeigten sich Makrophagen, wenige B-Zellen sowie multiple Foci von Plasmazell-Infiltraten. Degenerative Veränderungen manifestierten sich in Form eines fast vollständigen Zellverlustes. Vogt et al. folgerten, es handele sich insgesamt um eine starke und anhaltende immunologische Antwort auf das implantierte Allograft, die hauptsächlich durch T-Lymphozyten vermittelt werde. Sie verringere die Haltbarkeit des Grafts deutlich. Während im Organismus pädiatrischer Patienten eine persistierende zelluläre Abstoßung erfolge, sei sie bei Erwachsenen deutlich seltener und zudem in schwächerer Ausprägung festzustellen. Somit deutet ein Großteil der histomorphologischen und immunhistochemischen Untersuchungen der Literatur darauf hin, dass einer Immunreaktion des Empfängerorganismus eine entscheidende Rolle in der Degeneration implantierter Grafts zukommt.

Neben dieser primär zellulär getragenen Immunantwort ist auch eine humorale Abstoßungsreaktion gegen Homografts beschrieben worden. Goffin vermutete, eine humorale Abstoßung führe zu einer frühen Zerstörung unter anderem des Endothels und zu einer späteren Devitalisierung der Fibroblasten kryokonservierter Homografts (Goffin 1997). Allerdings hielt er trotzdem einen Funktionserhalt der Klappen für möglich, da die Extrazellulärmatrix weiterhin intakt bleibe. Smith et al. untersuchten die humorale Reaktion von Patienten auf die Implantation von Aorten-Homograftklappen in den linksventrikulären Ausflusstrakt (Smith et al. 1995). Die Allografts riefen eine Antikörper-Antwort 
hervor, die insbesondere Immunglobuline der Klasse IgG umfasste. Die Antikörper konnten über Zeitspannen von bis zu 15 Jahren nachgewiesen werden. Es ist anzunehmen, dass es auch unter den Bedingungen der vorliegenden Arbeit zu einer Produktion von Antikörpern kam. Diese These wird insbesondere gestützt durch den immunhistochemischen Nachweis von Plasmazellen.

\section{Entzündungsreaktion in den Rindervenengrafts}

Auf Grund der geringen Präparate-Anzahl von drei Rindervenengrafts war es schwierig, allgemeine Schussfolgerungen aus den Ergebnissen zu ziehen. Gemeinsam war den untersuchten Grafts, dass alle Präparate Entzündungszellen enthielten. Dabei wies die Adventitia die meisten inflammatorischen Zellen auf, in der Pseudointima fanden sich eher wenige. Immunhistochemisch ließen sich CD3-, CD4- und CD8-positive T-Zellen, Makrophagen, B-Lymphozyten und Plasmazellen nachweisen. Das Graftgewebe war unterschiedlich stark von Entzündung betroffen. Generell war die entzündliche Reaktion in den XenograftKlappen deutlich stärker ausgeprägt als in den Homograft-Klappen. In der bovinen Klappe des Präparats 11 zeigten sich vor allem im Graftgewebe Histiozyten und Fremdkörperriesenzellen. Dieser Befund ließ sich als Hinweis auf eine Abstoßung des Xenograft-Gewebes interpretieren (Vergleiche auch Ergebnisse bzgl. der Hancock-Conduits unter 4.5.2).

Es liegen vergleichsweise wenige Literaturangaben zur Histologie von Entzündungsreaktionen in Rindervenengrafts vor. Bisherige Studien beschränkten sich vornehmlich auf klinische Angaben. Mehrere Autoren beschrieben aber eine Fremdkörperreaktion in der Adventitia (Göber et al. 2005 und Shebani et al. 2006). Die Histiozyten und Fremdkörperriesenzellen hätten sich in unmittelbarer Nähe des Graft-Gewebes befunden (Boudjemline et al. 2003 und Dave et al. 2005). Zudem wiesen Göber et al., Dave et al. und Shebani et al. Infiltrate inflammatorischer Zellen wie Lymphozyten sowie Makrophagen und Fremdkörperriesenzellen in der Pseudointima an Conduit-Wand und Klappensegeln nach. Göber et al. vermuteten, die zelluläre Immunreaktion gegen das bovine Gewebe führe zu exzessiver Pseudointima-Bildung mit Stenosierung der Grafts.

\section{Entzündungsreaktion in den Hancock-Conduits und im PTFE-Graft}

Allen PTFE-enthaltenden Grafts war gemeinsam, dass sich den Kunststofffasern benachbart CD68-positive Makrophagen fanden. Diese lagen dort als einzelne Histiozyten, aber häufig auch als mehrkernige Riesenzellen vor (Vgl. Abb. 3.66). B- und noch zahlreicher T-Zellen ließen sich meist ebenfalls im PTFEMaterial dieses Conduit-Typs nachweisen. Im Vergleich mit den HancockConduits enthielt das klappenlose PTFE-Graft ohne porcines Gewebe insgesamt relativ wenige inflammatorische Zellen. In den Hancock-Conduits zeigte sich eine ausgeprägte Entzündungsreaktion, von der vornehmlich das Pseudointima-Gewebe betroffen war. Hier ließen sich T- und B-Lymphozyten sowie Histozyten und Fremdkörperriesenzellen identifizieren. Die inflammatorischen Zellen waren besonders zahlreich in der Nähe des Graft-Gewebes nachweisbar. Im porcinen Segment der Conduits selbst waren die Entzündungszellen zum Teil nur im Randbereich lokalisiert. Der Grund hierfür war möglicherweise, dass 
ein Eindringen der inflammatorischen Zellen in das Schweine-Gewebe verglichen mit der Fortbewegung in der Pseudointima erschwert war. In den porcinen Klappen fanden sich ebenfalls T-Lymphozyten, Histiozyten, Fremdkörperriesenzellen und B-Lymphozyten. Sie waren hier deutlich zahlreicher als in den Homograft-Klappen. Auch diese Beobachtung deutete auf eine Immunreaktion des Empfängerorganismus gegen das xenogene Gewebe hin.

Die Entzündungsreaktion des menschlichen Organismus auf porcine Xenografts ist vielfach untersucht worden. Bottio et al. beschrieben gruppierte und einzelne mononukleäre Entzündungszellen in porcinen Grafts (Bottio et al. 2003). Sie waren überwiegend direkt unter der Klappenoberfläche lokalisiert. Hieraus schlussfolgerten Bottio et al., die Zellen seien aus dem Blut eingewandert. Schreiber et al. wiesen in Conduits mit Schweine-Klappe eine chronische Entzündng mit Fremdkörperriesenzellen nach (Schreiber et al. 2006). Die Entzündungsreaktion war in der Pseudointima auf Höhe des porcinen klappentragenden Segments am stärksten ausgeprägt. Immunhistochemisch identifizierten Schreiber et al. in allen Conduits zahlreiche Makrophagen, weniger T-Lymphozyten (positiv für CD3, CD4 und CD8) sowie einige B-Lymphozyten (positiv für CD20). Die Entzündungsreaktion in den Klappen sei geringer ausgeprägt gewesen als im Gewebe der Conduit-Wand. Pearl et al. und Kim et al. dokumentierten ausgedehnte inflammatorische Infiltrate in der Pseudointima porciner Conduits (Kim et al. 2007 und Pearl et al. 2002). Histopathologisch wiesen sie in unmittelbarer Nähe des Schweine-Gewebes viele Fremdkörperriesenzellen und auch Plasmazellen nach. Darüber hinaus zeigten sich weniger Lymphozyten und Granulozyten. Pearl et al. vertraten die These, die Fremdkörperreaktion habe zu einer verstärkten Pseudointima-Bildung mit konsekutiver früher Stenose der porcinen Grafts geführt. Zwar sei es auch in untersuchten Homografts zur Formierung einer Pseudointima gekommen, jedoch in geringerer Ausprägung und mit langsamerem Fortschreiten als in porcinen Xenografts. Dies deckt sich mit der Beobachtung einer besonders ausgeprägten Bildung septierter und thrombotischer Pseudointima mit inflammatorischen Zellen in den Hancock-Conduits der vorliegenden Arbeit. Dieser könnte eine Abstoßung des porcinen Gewebes durch den Patientenorganismus zu Grunde liegen.

Rocchini et al. beobachteten in explantierten, mit Glutaraldehyd vorbehandelten porcinen Klappen histiozytäre Infiltrate sowie in drei von neun Präparaten zusätzlich Plasmazellen (Rocchini et al. 1981). In diesen Klappen ließen sich immunhistochemisch auch Immunglobuline nachweisen. Rocchini et al. vermuteten daher, eine Dysfunktion von Xenografts werde teilweise durch eine Immunreaktion ausgelöst. Manji et al. untersuchten die These, dass bei pädiatrischen Patienten mit ihrem im Vergleich zu Erwachsenen aktiveren Immunsystem eine Abstoßungsreaktion auf mit Glutharaldehyd vorbehandelte bovine und porcine Xenografts erfolge, die zu einer Verkalkung der Grafts führe (Manji et al. 2006). Als Modell für die Xenograft-Implantation verwandten sie junge Ratten, denen Aortengrafts von Meerschweinchen in die infrarenale Aorta implantiert wurden. Sie beobachteten eine ausgeprägte Entzündungsreaktion in den Xenografts, die die Klappen weniger betraf als Media und Adventitia. Immunhistochemisch wiesen sie Makrophagen und T-Zellen nach. Wie auch in den Präparaten dieser Arbeit lagen die Makrophagen in der Ad- 
ventitia dem Graft-Gewebe unmittelbar benachbart, die T-Lymphozyten hingegen fanden sich weiter peripher. Außerdem ermittelten Manji et al. einen deutlichen Anstieg der IgG-Titer der Ratten. Die Infiltration durch inflammatorische Zellen war mit einer Verkalkung verbunden. Auf der Grundlage ihrer Ergebnisse schlussfolgerten Manji et al., es komme generell zu einer zellulären und humoralen Abstoßung von glutaraldehydfixierten Xenografts. In der Folge verlören die Klappen ihre Funktionsfähigkeit. Die inflammatorischen Zellen der beiden äußeren Conduit-Schichten entstammten den Autoren zufolge dem extravaskulären System. Des Weiteren erklärten sie ähnlich wie Bottio et al., intravaskuläre Entzündungszellen adhärierten an den Klappensegeln und infiltrierten diese. Auf Grund des Blutflusses sei ein Einwandern der Zellen hier schwieriger.

\subsubsection{Entzündungsreaktion bei Conduit-Endokarditis}

Das Homograft 6 war nach 19 Jahren und sechs Monaten wegen Stenosierung explantiert worden. Zuvor hatte die Patientin unter rezidivierenden bakteriellen Endokarditiden gelitten. Laut OP-Bericht waren diese zum Zeitpunkt der Operation erfolgreich saniert. Dennoch wies das Präparat eine deutlich vermehrte Entzündungsreaktion auf, von der insbesondere die Adventitia betroffen war. Hier zeigten sich Lymphfollikel, deren Zellen immunhistochemisch als B- und T-Lymphozyten identifiziert werden konnten. Vermutlich waren die Lymphfollikel Ausdruck der Auseinandersetzung des Immunsystems der Patientin mit den Endokarditiserregern. Zudem ließ sich eine große Zahl CD68positiver Makrophagen - vor allem in der Pseudointima - nachweisen. Der Hancock-Conduit 14 wurde wegen Stenosierung, Insuffizienz und einer bakteriellen Endokarditis explantiert. Er enthielt weite Abschnitte septierter und thrombotischer Pseudointima mit massenhaft Granulozyten, Lymphozyten und Erythrozyten, die dem makroskopischen Befund von Eiter entsprachen.

Bakterielle Endokarditiden sind in der Literatur als eine relativ häufige Komplikation nach Implantation von Conduits im rechtsventrikulären Ausflusstrakt beschrieben und für alle drei in dieser Arbeit untersuchten Grafttypen dokumentiert. Sie manifestierten sich morphologisch beispielsweise als bakterielle Vegetationen und Thromben an den Klappensegeln (Schreiber et al. 2006). Butany et al. stellten fest, dass sich das Risiko für das Auftreten einer infektiösen Endokarditis über mehrere Jahre besonders für Patienten erhöhte, die vor Implantation des Conduits bereits von einer Endokarditis betroffen gewesen seien (Butany et al. 1999). Auch nach der kürzlich erfolgten Revision der Leitlinien für die Endokarditisprophylaxe gehören Patienten mit biologischem Klappenersatz - wie Patienten mit klappentragendem Conduit in Pulmonalisposition - ebenso wie Patienten mit durchgemachter Endokarditis zu der Gruppe der Hochrisikopatienten, bei denen bei drohender Bakteriämie unverändert eine prophylaktische Antibiotikagabe empfohlen wird (Wilson et al. 2007).

\subsubsection{Pseudointima-Bildung mit Stenosierung als Manifestation ei- ner Abstoßungsreaktion}

Bei einem Patienten erfolgte nach zehn Jahren und acht Monaten ein Austausch des stenosierten Pulmonalis-Homografts 2 gegen ein weiteres Pulmonalis- 
Homograft (3). Das zuerst implantierte (2) zeigte in der histopathologischen Untersuchung keine außergewöhnliche Entzündungsreaktion. Allerdings manifestierte sich im Conduit 3 klinisch bereits nach etwa drei Monaten eine zunehmende Stenosierung. Nach einem Jahr und zwei Monaten wurde eine Ballondilatation durchgeführt. Trotzdem musste das Graft nach weiteren fünf Monaten auf Grund der Stenose operativ entfernt werden. In der feingeweblichen Untersuchung zeigte sich im gesamten Präparat eine ausgeprägte Entzündungsreaktion. Die Pseudointima war an der Conduit-Wand und den Klappensegeln septiert und enthielt zahlreiche T-Zellen und Histiozyten. Die Adventitia wies ausgedehnte perivaskuläre T-Lymphozyten-Infiltrate sowie ebenfalls viele Makrophagen auf. Der ursprünglich zur Korrekturoperation verwendete Hancock-Conduit 14 eines anderen Patienten wurde wegen einer bakteriellen Endokarditis gegen ein Pulmonalis-Homograft (4) ausgetauscht. Dieses stenosierte jedoch rasch, so dass es bereits nach sechs Monaten explantiert werden musste. Intraoperativ erschien das Homograft laut OP-Bericht geschrumpft. Dieser Beschreibung entsprachen mikroskopisch Längsfalten des Conduits. Die Falten enthielten Pseudointima-Septen mit zahlreichen Granulozyten, T- und B-Lymphozyten und Makrophagen. Ausgeprägte Bereiche septierter Pseudointima und inflammatorische Zellen zeigten sich auch in den Klappenwinkeln des Präparats. Das Graft-Gewebe und besonders die Adventitia wiesen ebenfalls entzündliche Infiltrate auf. Es ließen sich Histiozyten, CD3- und CD8-positive T-Zellen sowie B-Lymphozyten und Plasmazellen nachweisen. Die Anamnesen der zwei zuletzt beschriebenen Patienten ähnelten sich insofern, als dass bei beiden das zweite implantierte Homograft innerhalb kurzer Zeit eine derart ausgeprägte Stenose aufwies, dass eine Explantation erforderlich wurde. Histologisch zeigten sich jeweils auffällig viel septierte Pseudointima und zahlreiche inflammatorische Zellen. Möglicherweise lag dieser Reaktion des Patientenorganismus eine erworbene Immunität gegenüber den Implantaten zu Grunde. Als mögliche Einflussfaktoren auf die Entzündungsreaktion in Conduits sind unter anderem das Patientenalter bei Implantation und die Blutgruppenkompatibilität diskutiert worden. Einige Autoren schlugen als Maßnahme gegen Entzündung, Abstoßung und Degeneration eine immunsuppressive Therapie, beispielsweise mit Cortison, vor (Manji et al. 2006, Neves et al. 1995 und Yankah et al. 1995b). In der Literatur wird vielfach auf die möglicherweise erhöhte Immunogenität von Conduits durch erhaltenes Endothel hingewiesen, die über Abstoßungsreaktionen des Empfängers zu einer beschleunigten Degeneration der Implantate führe (Erez et al. 2001 und Fischlein et al. 1995).

\subsection{Thrombenbildung}

Intravasale Blutgerinnsel können gemäß der Virchow'schen Trias auf Grund von Gefäßwand-Veränderungen, Störungen der Hämodynamik oder Änderungen der Blutzusammensetzung entstehen. Thromben setzen sich zusammen aus Fibrinnetzen mit Erythrozyten und Leukozyten sowie Thrombozytenaggregaten. Wie oben dargestellt, wies die Mehrzahl der untersuchten Präparate aufgelockerte Pseudointima-Bereiche auf, in denen sich immunhistochemisch teilweise Fibrin anfärben ließ. Diese thrombotische Pseudointima glich vielfach Unebenheiten der Oberfläche der Lumenzirkumferenz aus und dürfte somit einen 
laminaren Blutfluss durch den Conduit gefördert haben. Im Gegensatz hierzu enthielt das Rindervenengraft 11 (Implantationszeit 2 Jahre und 2 Monate) als einziges Präparat zusätzlich einen fibrinreichen parietalen Thrombus, der sich deutlich in das Lumen hineinwölbte. Das Gerinnsel wies die typische aufgelockerte Struktur mit Fibrinsepten, amorpher Substanz, Erythrozyten und inflammatorischen Zellen auf. Da der Thrombus von der Graftwand aus in das Lumen hineinragte, ist davon auszugehen, dass er in situ zu einem turbulenten Blutfluss und einem somit erhöhten Risiko der Entstehung weiterer Thromben geführt hatte. Der wandständige Thrombus wies Zeichen einer beginnenden Organisation auf, denn er war von Endothel bedeckt und enthielt im Inneren histiozytäre Zellen. Seine äußeren Anteile boten das Bild bindegewebig organisierter Pseudointima. Die im Zentrum des Thrombus enthaltenen Verkalkungsareale deuteten ebenfalls darauf hin, dass es sich um einen länger bestehenden Thrombus handelte.

Die Ausbilung von Thromben ist als typische Komplikation nach Implantation von Rindervenengrafts mehrfach in der Literatur diskutiert worden (Boudjemline et al. 2003 und Shebani et al. 2006). Tiete et al. vermuteten als Ursache einen langsamen Blutfluss durch den im Verhältnis zur nativen Situation großen Klappendurchmesser (Tiete et al. 2004). Shebani et al. erklärten, die Klappensegel der Rindervenengrafts seien tiefer als menschliche Semilunarklappen, woraus sich möglicherweise eine Prädisposition für Thrombosen ergäbe. Im Zusammenhang mit im rechtsventrikulären Ausflusstrakt implantierten Homografts wurde das Auftreten von Thromben ebenfalls beschrieben. Goffin beobachtete makroskopisch sichtbare Thromben auf der arteriellen Oberfläche einer Pulmonalklappe 21 Tage post implantationem (Goffin 1997). Sie seien teilweise organisiert gewesen. Auch bei Hancock-Conduits sind Thromben in der Literatur beschrieben (Mair et al. 1978).

Chang et al. untersuchten Rindervenengrafts in einem Hundemodell und kamen zu dem Schluss, mit Glutaraldehyd fixierte Implantate seien potentiell thrombogen, weil durch die zytotoxische Wirkung des Mittels eine Migration von Endothelzellen des Empfängers für mindestens sechs Monate unterdrückt werde (Chang et al. 2001). Boudjemline et al. empfahlen vor der Implantation ein ausführliches Spülen von Rindervenengrafts, um das Glutaraldehyd auszuwaschen und so dessen Zytotoxizität zu reduzieren (Boudjemline et al. 2003). Von verschiedenen Arbeitsgruppen und Kliniken werden unterschiedliche medikamentöse Regime zur Vermeidung von Thrombusbildung in Conduits angewendet. Weit verbreitet ist die Thrombozytenaggregationshemmung durch ASS (Brown et al. 2006, Corno et al. 2002, Dave et al. 2005, Göber et al. 2005, Ishizaka et al. 2003, Meyns et al. 2004, Pearl et al. 2002, Shebani et al. 2006, Tiete et al. 2004 und Yankah et al. 1995a), wie sie in den Leitlinien zur Diagnostik und Therapie der Deutschen Gesellschaft für Pädiatrische Kardiologie empfohlen (Schmaltz 2007) und auch in der hiesigen Kinderherzchirurgie durchgeführt wird. Eine weitere Möglichkeit der antithrombogenen Therapie ist eine Antikoagulation durch Vitamin-K-Antagonisten (Schreiber et al. 2006). Um den Patienten dagegen die dauerhafte Einnahme von Medikamenten zu ersparen, wurden unterschiedliche Vorbehandlungsmethoden der Grafts erprobt (Ichikawa et al. 1997 und Sarkar et al. 2007). So sind beispielsweise Vorteile hinsichtlich der Vermeidung thrombembolischer Ereignisse 
durch eine Oberflächenbeschichtung mit Heparin für verschiedene kardiovaskuläre Implantate dokumentiert worden (Lin et al. 2004). Für Xenografts, die bislang standardmäßig mit Glutaraldehyd fixiert werden, könnten alternative Vorbehandlungsmedien, wie das von Chang et al. empfohlene Genipin, möglicherweise zu einem längeren Funktionserhalt der Conduits führen (Chang et al. 2001).

\subsection{Verkalkung}

Die Begriffe Verkalkung, Kalzifkation und Kalzifizierung bezeichnen die Ablagerung von mineralischen Calcium-Phosphat-Verbindungen in Kristallform. Hierbei handelt es sich im Zusammenhang mit Gefäßimplantaten vorwiegend um Hydroxylapatit (Hayabuchi et al. 2007, Park et al. 2001 und Valente et al. 1985). Aus der Mineralisierung resultiere ein Verlust an Flexibilität in biologischen Geweben, was zu Degeneration und mechanischem Versagen von Implantaten führe. So ist eine kalzifizierende Stenose als wichtige Indikation zur Conduit-Explantation für porcine Grafts und insbesondere für Homografts oft beschrieben worden (Collett und Canfield 2005 und Mitchell et al. 1995). Zu einer kalzifizierenden Stenose komme es besonders häufig, wenn Conduits in den rechtsventrikulären Ausflusstrakt pädiatrischer Patienten implantiert würden (Cleveland et al. 1992 und Vivie et al. 1981). Auf Grund der hohen klinischen Relevanz von Kalzifizierungsprozessen in kardiovaskulären Implantaten liegen zahlreiche Untersuchungen zu dieser Thematik vor. In vielen Fällen wurde eine Verkalkung der Conduit-Wand dokumentiert. Die meisten Studien ergaben im Vergleich eine geringere Kalzifizierung der Klappensegel (Koolbergen et al. 2002b, Mitchell et al. 1995 und Vogt et al. 1999).

In der vorliegenden Arbeit wies die Mehrzahl der untersuchten Grafts Kalkareale auf. Bei den Homografts und PTFE-Conduits war eine deutliche Korrelation zwischen Ausprägung der Verkalkung und Implantationsdauer feststellbar. Generell zeigten sich sowohl intra- als auch extrazelluläre Verkalkungen. Zum Teil ließen sich die histologischen Strukturen wie Fasern und Zellen noch abgrenzen, teilweise lag jedoch eine flächige Verkalkung vor, die sich in der von-Kossa-Färbung als homogen schwarze Fläche demarkierte. Vereinzelt fanden sich in den Kalkherden Entzündungszellen und Erythrozyten.

Laut Daten der Literatur gelten als Ausgangspunkte von Kalzifizierung lysierte Zellen und Membranfragmente (Bottio et al. 2003, Mitchell et al. 1995 und Valente et al. 1985). Im weiteren Verlauf des Verkalkungsprozesses seien zusätzlich Kollagenfasern betroffen. Eine detaillierte Beschreibung von Verkalkungprozessen lieferten Webb et al. für klappenlose Aortenallografts in Ratten (Webb et al. 1992). In subdermal implantierten Grafts ließen sich erste kleine Kalkablagerungen nach 24 bis 48 Stunden in der Media nachweisen. Sie waren mit Kernen, Zytoplasma oder Zellmembranen nekrotischer glatter Muskelzellen verbunden. Im weiteren Verlauf (7 Tage) waren auch die Fasern von Verkalkung betroffen. Schließlich (3 Wochen) zeigten sich zahlreiche konfluierende, knotige Kalkherde. Insgesamt überwog dabei die zellgebundene gegenüber der extrazellulärmatrixassoziierten Verkalkung. Die Morphologie dieser rasch fortschreitenden Verkalkung in dem subdermalen Modell sei vergleichbar mit Ergebnissen aus der klinischen Erfahrung und ähnlich den Veränderungen in 
mit Glutaraldehyd vorbehandelten Schweine-Klappen. Bei der Interpretation der Studienergebnisse von Webb et al. muss beachtet werden, dass die Aortengrafts nicht - wie bei Patienten - in den Blutkreislauf integriert und von Blut durchflossen waren, woraus sich sicherlich Unterschiede in der Gewebereaktion ergeben.

Innerhalb der Klappen der von uns untersuchten Homografts und HancockConduits waren Verkalkungsherde gehäuft an der Basis lokalisiert. Klinisch könnte dies Ursache einer insuffizienten Klappenfunktion gewesen sein. Für mindestens einen der Hancock-Conduits mit verkalkter Klappenbasis in dieser Serie war als Explantationsgrund eine Insuffizienz angegeben. In unseren Präparaten bestanden zwischen den drei verschiedenen Conduit-Typen Unterschiede in Ausmaß und Lokalisation der Verkalkung, wie im Folgenden ausgeführt wird.

\subsubsection{Verkalkung der Homografts}

Acht der zehn Homografts waren verkalkt, was jeweils schon makroskopisch erkennbar war. Mikroskopisch war vor allem die Pseudointima betroffen. Kalkherde waren außerdem in mehreren Präparaten in der ursprünglichen Media enthalten, darunter alle drei gestenteten Grafts. Das Pseudointima-Gewebe wies bei diesen Explantaten vor allem in unmittelbarer Nähe der Stentstreben Kalkareale auf. In einigen Präparaten - auch ohne Stent - setzte sich die schichtübergreifende Verkalkung bis in die Adventitia hinein fort. Jeong et al. untersuchten degenerative Prozesse an Aortenallografts nach Implantation in den rechtsventrikulären Ausflusstrakt von Hunden (Jeong et al. 1976). Sie dokumentierten in der Conduit-Wand einen frühen Zellverlust und eine Ausdünnung der Fasern. Calcium lagerte sich ab Tag 16 nach Implantation ab, so dass ab Tag 33 in allen Grafts eine Verkalkung von Aortenwand und Klappenbasis feststellbar war. Die Klappensegel seien hingegen kaum verkalkt gewesen. Generell sei die Kalzifizierung auf azelluläre Areale der Grafts beschränkt gewesen. Die Autoren folgerten, die wichtigste Voraussetzung für einen langen Funktionserhalt ohne Verkalkung von Allografts sei der Erhalt der Zellen. In der vorliegenden Arbeit waren die Conduit-Wände und die Klappenbasis ebenfalls stärker von Kalzifikation betroffen als die Klappensegel. Allerdings waren Kalkherde auch in Bereichen der Präparate zu erkennen, in denen Zellen erhalten waren (Vgl. beispielsweise Abb. 3.11).

Es liegen widersprüchliche Ergebnisse vor, ob die Homograftfunktion durch den Klappentyp, also die Herkunft der Klappe aus dem pulmonalen oder aortalen Ausflusstrakt, beeinflusst wird (Bando et al. 1995, Baskett et al. 1996, Cleveland et al. 1992, Koolbergen et al. 2002a, Lange et al. 2001, Shaddy und Hawkins 2002 und Tweddell et al. 2000). Javadpour et al. untersuchten radiologisch und echokardiografisch Patienten, deren rechtsventrikulärer Ausflusstrakt mit einem Homograft rekonstruiert worden war (Javadpour et al. 2002). 20\% der Conduits wiesen eine Verkalkung auf. Sie entwickelte sich innerhalb von maximal 18 Monaten und betraf ausschließlich Aorten-Homografts. Javadpour et al. kamen zu dem Ergebnis, dass Pulmonalisgrafts in dieser Hinsicht den Aortengrafts als Implantat im rechtsventrikulären Ausflusstrakt überlegen seien. Diesen Schluss zogen auch Yankah et al. auf Grundlage von Untersuchungen an 
aus dem rechtsventrikulären Ausflusstrakt von Kindern explantierten Homografts (Yankah et al. 1995a). Sie ermittelten als Freiheit von Wandverkalkungen nach 20 Monaten Werte von 19\% für Aortengrafts versus 100\% für Pulmonalisgrafts. Mögliche Erklärungen für die höhere Resistenz von Pulmonalisgrafts gegen frühe Wandkalzifizierung basierten auf der Beobachtung, dass Verkalkung meist mit elastischen Fasern assoziiert sei und zudem von der Gesamtmenge des Gewebecalciums abhänge (Livi et al. 1987). Pulmonalarterien enthielten nur 50 bis $60 \%$ der Menge an elastischen Strukturen von Aortengrafts. Auch die biochemisch nachweisbaren Calciumwerte in Pulmonalis-Mediagewebe betrügen durchschnittlich weniger als die Hälfte von Werten der Aorta. In der vorliegenden Arbeit manifestierte sich zwischen Aorten- und Pulmonalis-Homografts histologisch kein Unterschied in der Verkalkung oder anderen degenerativen Veränderungen. Möglicherweise wäre hierfür eine größere Anzahl von Präparaten erforderlich gewesen.

Bezogen auf die frühe Kalzifikation von Homografts identifizierten Yankah et al. neben dem Grafttyp (siehe oben) als weiteren Risikofaktor ein junges Patientenalter (Yankah et al. 1995a). Sie sahen die Ursache in einer Immunantwort des Empfängers gegen das Graft und vermuteten, die Abstoßungsreaktion und Verkalkung könnten durch eine Steroidmedikation vermindert werden. Diverse weitere Autoren vertraten die These, dass der Verkalkung von Allografts eine immunologische Reaktion des Empfängerorganismus zugrunde liege (Mathieu et al. 2003, Santini et al. 1996 und Schulick et al. 1998). Brockbank et al. vermuteten als Grund der Kalzifikation von Homograftklappen ihre Vorbehandlung (Brockbank et al. 2000). Durch konventionelle Kryokonservierung bildeten sich extrazellulär zahlreiche Eiskristalle. Diese schädigten die Extrazellulärmatrix und prädisponierten die Klappen so für eine Verkalkung. Da die in der vorliegenden Arbeit analysierten Homografts allesamt der gleichen Vorbehandlung unterzogen wurden, konnten wir keine Aussagen zu möglichen Vorzügen der von Brockbank et al. alternativ vorgeschlagenen Vitrifizierung treffen.

\subsubsection{Verkalkung der Hancock-Conduits und des PTFE-Grafts}

Im Vergleich zu den Homografts waren die Verkalkungsareale in der Pseudointima der von uns untersuchten Hancock-Conduits kleinflächiger und weniger zahlreich. Allerdings wies das Schweine-Gewebe am klappentragenden Segment in neun der zehn Grafts Kalkeinlagerungen auf. Diese umfassten sowohl kleinere verkalkte Areale als auch mehrfach flächige Verkalkungsherde. Insgesamt war die Kalzifikation des porcinen Graft-Gewebes stärker ausgeprägt als in den beiden anderen Conduit-Typen. Auch in der Literatur wird die Verkalkung porciner Grafts als ein die Klappenfunktion beeinträchtigender Faktor beschrieben (Butany et al. 1999 und Valente et al. 1992). Milano et al. identifizierten die kalzifizierende Degeneration porciner Grafts als progressives, in Beginn und Ausmaß zeitabhängiges Phänomen, das die Hauptursache von Klappenversagen sei (Milano et al. 1984). Bottio et al. betonten die Bedeutung mechanischer Belastung in der Kalk-Entstehung. Beispielsweise seien die Klappenkommissuren dem Blutfluss besonders stark ausgesetzt und verkalkten daher früh. Mitchell et al. beschrieben, kryokonservierte Homograft-Klappen 
seien resistenter gegenüber Verkalkungsprozessen als porcine Klappen. Sie vermuteten, durch stärkere Autolyse in den kryokonservierten Homografts verblieben geringere Mengen an Zellschrott, der im weiteren Verlauf verkalken könne. Bei der Glutaraldehydbehandlung würden dagegen mehr Zellbestandteile der Schweine-Klappen erhalten. Es wurden Versuche unternommen, Xenografts zu entwickeln, die Mineralisierungsprozessen weniger stark unterliegen (Jashari et al. 2001, Ota et al. 2007 und Valente et al. 1992). Durch bestimmte Vorbehandlungsmethoden porciner Grafts sei beispielsweise eine Verlängerung der Implantationszeit bis zum strukturellen Klappenversagen um zwei bis drei Jahre möglich (Bottio et al. 2003 und Butany et al. 1999).

Auch das PTFE-Material zeigte sich in der vorliegenden Arbeit verkalkt. Der klappenlose PTFE-Conduit war nach einer Implantationszeit von 15 Jahren und acht Monaten stärker verkalkt als die Hancock-Conduits. Er wies eine hart-brüchige Konsistenz auf und enthielt schichtübergreifend Areale flächiger Verkalkung, die auch die Adventitia betrafen. Eine ausgeprägte, generalisierte Verkalkung von PTFE-Grafts wurde in der Literatur beschrieben (Boethig et al. 2005, Ehler et al. 1990 und Molina et al. 1995). Hayabuchi et al. ermittelten eine Kalzifikationsrate von $81 \%$ bei den von ihnen untersuchten zur RVOTRekonstruktion implantierten PTFE-Grafts (Hayabuchi et al. 2007). Park et al. verglichen in einem in vitro Modell die Kalzifizierungstendenz dreier synthetischer Materialien, die zur Herstellung von Gefäßprothesen genutzt werden (Park et al. 2001). PTFE wies dabei deutlich höhere Calciumwerte auf als Polyurethan und Silikon. Dies führten die Autoren auf die rauhe Oberfläche des PTFE-Gewebes zurück. Auf dieser laufe der typische Kalzifizierungsprozess die Akkumulation von Calcium- und im Verlauf zunehmend auch Phosphationen - besonders stark und schnell ab. Demgegenüber stünden als positive Aspekte von PTFE die geringe Thrombogenität, gute mechanische Eigenschaften und eine lange Haltbarkeit.

\subsubsection{Verkalkung der Rindervenengrafts}

Eine Verkalkung von Rindervenengrafts ist in klinischen Studien im Rahmen kurz- und mittelfristiger Beobachtungszeiträume generell eher selten und ohne funktionelle Beeinträchtigung der Conduits dokumentiert worden (Corno et al. 2004 und Shebani et al. 2006). Unter den drei von uns untersuchten Rindervenengrafts wies dasjenige mit der mittleren Implantationsdauer von zwei Jahren und zwei Monaten ausgeprägte Verkalkungen auf. Diese betrafen die Pseudointima und das Graftgewebe von Conduit-Wand und -Klappe sowie den wandständigen Thrombus des Präparates. In der allerdings sehr kleinen Gruppe der Rindervenengrafts zeigte sich somit keine Abhängigkeit zwischen Implantationszeit und Ausprägung der Mineralisierung. Manji et al. vermuteten generell eine Abstoßungsreaktion gegen Xenografts und erklärten, die Implantate verkalkten dem Ausmaß der Entzündung entsprechend (Manji et al. 2006). Ähnlich wie Yankah et al. diskutierten sie daher eine Therapie mit Steroiden nach Implantation eines biologischen Conduits (Yankah et al. 1995b). 


\subsubsection{Mit Verkalkung assoziierte Befunde}

In der Literatur gibt es über die oben zitierten Ergebnisse hinaus Thesen bezüglich der Kalzifizierung von Gefäßen, die kontrovers diskutiert werden. Mehrfach wird der Vergleich zwischen Verkalkungsprozessen und dem Vorgang der Ossifikation gezogen (Mathieu et al. 2003). Mohler et al. stellten eine Verkalkung von $83 \%$ der von ihnen untersuchten 347 orthotopen humanen Aortenklappen fest (Mohler et al. 2001). Von diesen verkalkten Arealen entsprachen 13\% reifem lamellärem Knochen mit hämatopoietischen Elementen und aktivem Knochenumbau. Die Autoren vermuteten Reparaturmechanismen als Ursache. In allen Klappen mit Ossifikation zeigten sich Neovaskularisation sowie vielfach Entzündungszell-Infiltrate. Mohler et al. schlussfolgerten, dass der Verkalkung und Ossifikation orthotoper Klappen entzündliche Prozesse zugrunde lägen. Auch Rajamannan et al. werteten die Kalzifikation ortsständiger menschlicher Aortenklappen als aktiven, gesteuerten Prozess, der der Knochenbildung im Skelettsystem ähnele (Rajamannan et al. 2003). Hierbei trete ein osteoblastenähnlicher Zelltyp auf. Collet et al. stellten fest, Verkalkung sei assoziiert mit Neovaskularisation, wobei diese eine notwendige, jedoch nicht hinreichende Voraussetzung für die Mineralisierung sei (Collett und Canfield 2005). Auch Perizyten könnten zu vaskulärer Kalzifikation beitragen (Farrington-Rock et al. 2004). Des Weiteren wird in der Literatur die chondroide Metaplasie (siehe unter 4.3.4) als mögliche Vorstufe von Verkalkung diskutiert (Qiao et al. 1995).

\subsection{Taschenklappen}

Die Taschenklappen von Aorta und Pulmonalarterie bestehen aus jeweils drei taschenförmigen Klappensegeln. Bei Venenklappen handelt es sich um Intimaduplikaturen nach Art der Taschenklappen. Die Klappen der untersuchten bovinen Venengrafts weisen ebenfalls jeweils drei Segel auf. Taschenklappen erfügen über ein bindegewebiges Grundgerüst, das sich durch seinen hohen Gehalt an Kollagenfasern und elastischem Gewebe auszeichnet (Armiger 1995, Goffin 1997 und Mitchell et al. 1995). Die Segel sind in drei Schichten gegliedert. An der zur Gefäßwand gerichteten Oberfläche befindet sich die Fibrosa aus straffem kollagenen Bindegewebe. Die elastinreiche Lage an der ventrikulären Oberfläche wird als Ventrikularis bezeichnet. Die dazwischen gelegene Schicht lockeren Bindegewebes heißt Spongiosa. Diese ist reich an Hyaluronan und Proteoglykanen. Der Klappenanulus hat eine doppellagige Struktur: Der dem Sinus benachbarte Ring ist fibrös, der untere spongiös. Im basalen Teil der Ventrikularis können sich wenige schmale Bündel glatter Muskelzellen befinden. Nur im Anulus und gelegentlich in der Basis der Klappe kommen kleine Gefäße vor. Ansonsten sind die Klappensegel gefäßfrei (Rajani et al. 1998). An den Oberflächen der Klappen findet sich eine einlagige Schicht von Endothelzellen (Endokard).

In der vorliegenden Arbeit konnte keine native Klappe untersucht werden. Da die histologischen Befunde der explantierten Conduits jedoch vielfach mit verfügbaren Literaturangaben übereinstimmten, ist davon auszugehen, dass die untersuchten Grafts den in der Literatur beschriebenen auch vor Implan- 
tation strukturell geähnelt hatten. Die Taschenklappen der drei analysierten Grafttypen wiesen eine vergleichbare histologische Struktur auf. Ihr bindegewebiges Grundgerüst bestand vor allem aus Fasern und enthielt nur wenige Zellen. Eine Abgrenzung von Ventrikularis, Spongiosa und Fibrosa war meist nicht möglich. Ein fortschreitender Verlust von Bindegewebszellen und ein Verwischen der normalen histologischen Struktur nach Klappenimplantation ist auch in der Literatur dokumentiert worden (Koolbergen et al. 2002b, Mitchell 1995 und Neves et al. 1995). An den Zellen im Graft-Gewebe waren erwartungsgemäß keine muskelzelltypischen Antigene identifizierbar. Nur in der Pseudointima der porcinen Klappen ließen sich unter diesen zum Teil Vimentin- und SMA-positive fibromuskuläre Zellen anfärben. Die Pseudointima von Homograft- und Schweine-Klappen enthielt stellenweise Kapillaren sowie Erythrozyten. Zu kleineren Einblutungen in das Gewebe könnte es möglicherweise intraoperativ gekommen sein. An Klappen aller drei Conduit-Typen ließ sich immunhistochemisch Endothel nachweisen. In verfügbaren Studien sind hingegen wenige oder keine Endothelzellen an den explantierten Taschenklappen beschrieben (Koolbergen et al. 2002b und Neves et al. 1995). Degenerative Veränderungen fanden sich in allen Grafttypen insbesondere in Form von Kalkarealen. Diese betrafen sowohl das Graft- als auch das PseudointimaGewebe der Taschenklappen. Die Kalzifikation war in den porcinen Klappen stärker ausgeprägt als in den beiden anderen Grafttypen und zum Teil vermehrt basisnah lokalisiert. Eine Verkalkung von Conduit-Wand und -Klappen ist auch in der Literatur oft dokumentiert und in dieser Arbeit unter 4.7 eingehender diskutiert. Trotz dieser und anderer zeitabhängiger Beeinträchtigungen der Funktion von Conduit-Klappen wird empfohlen, eine Implantation von klappenlosen Conduits nach Möglichkeit zu vermeiden (Boethig et al. 2005, Stark 1998 und Yuan et al. 1998). Das Fehlen einer Klappe wirke sich hämodynamisch äußerst ungünstig aus.

\section{$4.9 \quad$ Media}

Das Graft-Gewebe der Homografts und der Rindervenengrafts bestand hauptsächlich aus der ursprünglichen Gefäßmedia. In den Hancock-Conduits war in den PTFE-Schlauch ein klappentragendes Segment aus porcinem Gewebe eingenäht, dessen Hauptkomponente ebenfalls durch Media-Gewebe der Schweine-Aorta gebildet wurde. Die großen herznahen Arterien erfüllen Windkesselfunktion und zählen somit zur Gruppe der Arterien vom elastischen Typ. Deren Media besteht aus konzentrischen elastischen Membranen und glatten Muskelzellen (SMCs), die in abwechselnden Lagen geschichtet sind. Membrana elastica interna und externa sind in diesem Arterientyp nicht klar von den übrigen elastischen Lamellen abgrenzbar. Des Weiteren finden sich Kollagenfasern und amorphe Grundsubstanz. Venen enthalten zwischen den glatten Muskelzellen der Media ebenfalls reichlich elastische und kollagene Fasern.

Wie für native Arterien beschrieben, wiesen das Media-Gewebe der untersuchten Homografts und die Segmente porcinen Gewebes eine bindegewebige Struktur auf. Es zeigten sich zahlreiche gewellte elastische und kollagene Fasern, zwischen denen sich fibromuskuläre Zellen befanden. Auch in den bovinen Venengrafts bestand das Gewebe aus gewellten Fasern. Teilweise konnten diese 
als elastische und kollagene Fasern identifiziert werden. Sie waren zum Teil von vergleichsweise viel amorpher Substanz umgeben. Zudem zeigten sich fibromuskuläre Zellen. In beiden Xenograft-Geweben wiesen die fibromuskulären Zellen ein Antigenexpressionsmuster auf, wie es auch in Mediazellen humaner Grafts vorkam. Dies könnte darauf hindeuten, dass Zellen des Empfängers in das Graftgewebe eingewandert sind. Andererseits ist denkbar, dass sowohl menschliche Zellen als auch bovine und porcine Zellen generell positiv für die verwandten Antikörper färben. Unabhängig davon lässt sich feststellen, dass die Graft-Media aller verwendeten Conduit-Typen vitale Zellen enthielt. Im Schweine-Gewebe einiger Hancock-Conduits waren inflammatorische Zellen ausschließlich marginal lokalisiert (Vgl. Abb. 3.65). Die Ursache hierfür könnte darin liegen, dass die Bindegewebsstruktur der porcinen Media eine Zellmigration erschwert. So beschrieben Shi et al. für Koronararterien von Schweinen, eine intakte Gefäßmedia diene als Barriere, die eine Einwanderung von Zellen verhindere (Shi Y et al. 1996).

In den untersuchten Homografts, die nicht interventionell mit einem Stent versehen worden waren, ließ sich eine histomorphologische Zweiteilung des Graft-Gewebes erkennen. Lumenseitig waren die Fasern in den verschiedenen Färbungen klar abgrenzbar. Weiter in Richtung Adventitia fand sich hingegen wesentlich mehr amorphe Substanz bei zugleich deutlich schlechter abgrenzbaren Faserstrukturen. Dieser Befund war in der verfügbaren Literatur bisher so nicht beschrieben. Die gestenteten Conduits wiesen keine histologische Zweiteilung des Graft-Gewebes auf. Möglicherweise war dies ein Hinweis auf eine auch in der Literatur dokumentierte Mediaschädigung durch die Angioplastie. So zeigten Aikawa et al., dass humane Koronararterien nach Dilatation Einrisse der Gefäßwand bis zur Media aufwiesen (Aikawa et al. 1997). Eine interventionelle Aufdehnung von Homografts könnte also eine Störung der üblichen Mediastruktur zur Folge haben. Darüber hinaus zeigten Untersuchungen, dass eine Schädigung der Media zu vermehrter Gewebeneubildung an den Orten der Verletzung führte (Farb et al. 2002). Somit könne eine übermäßige Dilatation von Gefäßen die Pseudointima-Bildung verstärken und zu erneuter Stenosierung führen. In den drei untersuchten Rindervenengrafts war die morphologische Zweiteilung des Graft-Gewebes in Bereiche mit stärker und weniger deutlich gewellten Fasern uneinheitlich. In der Literatur wurde dieser Befund nicht dokumentiert. Somit blieb auch für diesen Conduit-Typ unklar, ob es sich um ein Artefakt oder beispielsweise eine durch die Präparation verursachte, reproduzierbare Veränderung handelte.

Mit den verwandten Methoden ließen sich Intima und Media des Graft-Gewebes nicht klar voneinander abgrenzen. Histomorphologische Ähnlichkeit erklärte auch die Schwierigkeit, insbesondere in einigen Homografts die neu gebildete Pseudointima eindeutig vom Graft-Gewebe zu differenzieren.

\subsection{Adventitia}

Die Adventitia als äußere bindegewebige Schicht eines Gefäßes setzt sich vor allem aus Fibroblasten, Kollagenfasern, elastischen Fasern und Proteoglykanen zusammen. Sie enthält neben den Vasa vasorum auch Lymphgefäße, Nerven und Fettzellen. In den untersuchten Grafts ähnelte sich die histologische Struk- 
tur der Adventitia in den drei Conduit-Typen. Sie wies einen bindegewebigen Aufbau aus Kollagenfasern und fibromuskulären Zellen sowie zahlreiche kleine Gefäße auf. Möglicherweise waren in den humanen und bovinen Conduits bei Implantation Anteile der Adventitia enthalten, wie es bei dem von uns untersuchten nicht-implantierten Kontroll-Homograft der Fall war. Die Übergänge zwischen ursprünglicher und neu entstandener Adventitia waren nicht sicher abgrenzbar. Es war meist eine ausgeprägte Entzündungsreaktion in der Adventitia nachweisbar, wie sie auch in der Literatur für verschiedene Grafttypen beschrieben wurde (Boudjemline et al. 2003 und Legare et al. 2000). Dieser Befund lässt sich vereinbaren mit der Feststellung von Anderson et al., Makrophagen steuerten Wachstum und Proliferation von Fibroblasten (Anderson und Miller 1984). Auf diese Weise induzierten sie auch eine Kollagenproduktion im Sinne einer Gewebereparatur während chronischer Entzündungen. Folglich könnte durch die beobachtete Entzündungsreaktion eine Verbreiterung der Adventitia der Conduits durch Bildung kollagenen Bindegewebes gefördert worden sein. 


\section{Kapitel 5}

\section{Zusammenfassung}

In der vorliegenden Arbeit wurde eine humane Präparate-Serie von 24 aus dem rechtsventrikulären Ausflusstrakt explantierten Conduits ausgewertet. Während in der Literatur überwiegend klinische Daten verfügbar sind, war es unser Ziel, die Biokompatibilität von Homografts, Rindervenengrafts und Hancockbzw. PTFE-Conduits mit Hilfe histologischer und immunhistochemischer Methoden zu vergleichen.

Bei der Analyse der drei verschieden Conduit-Typen zeigte sich lumenseitig durchgehend neu gebildetes Gewebe. Dabei fand sich eine vollständige Endothelialisierung der blutzugewandten Oberflächen. Ebenso enthielten alle Präparate Pseudointima-Gewebe, das sich aus kollagenen und elastischen Fasern sowie fibromuskulären Zellen zusammensetzte. Diese Zellen wiesen teilweise bereits nach einer Implantationszeit von sechs Monaten bei Färbungen mit Antikörpern gegen muskelzelltypische Antigene ein Expressionsmuster höher differenzierter Muskelzellen auf. Die Entwicklung der bindegewebigen Pseudointima verlief über Zwischenstufen septierter und thrombotischer Strukturen, die in ihrem Vorkommen abhängig von den rheologischen Bedingungen in den Conduits waren: In Bereichen mit verlangsamtem oder turbulentem Blutfluss fand sich besonders ausgedehnt septiertes und thrombotisches PseudointimaGewebe.

Die Pseudointima an den Graftoberflächen einschließlich der Klappen enthielt neu gebildete Kapillaren mit lumenseitigem Endothel und zirkulär angeordneten fibromuskulären Zellen. Diese Kapillarwand-Zellen zeigten ein fortgeschritteneres Differenzierungsmuster als die fibromuskulären Zellen in der umgebenden Pseudointima.

In allen Präparaten war unabhängig von der Implantationszeit eine signifikante Entzündungsreaktion nachweisbar. Sie war in den Wandstrukturen der Conduits deutlicher ausgeprägt als im Bereich der Klappen. Perivaskulär um neu gebildete kleine Gefäße fanden sich inflammatorische Zellen gehäuft. Mit den angewandten Antikörpern gegen CD-Antigene ließen sich vor allem TLymphozyten und Makrophagen identifizieren. B-Lymphozyten und Plasmazellen waren in geringerer Zahl vorhanden. CD68-positive Histiozyten und Fremdkörperriesenzellen fanden sich insbesondere in der Nähe synthetischer Fremdmaterialien. Im Vergleich der drei Conduit-Typen zeigte sich im porcinen Gewebe die stärkste Entzündungsreaktion, was als Immunreaktion des Empfängerorganismus gegen das xenogene Gewebe zu werten ist. Die inflam- 
matorischen Reaktionen waren insgesamt in den Xenografts deutlicher ausgeprägt als in den Homograft-Conduits. Zudem hatte die Entzündungsreaktion eine verstärkte Bildung septierter und thrombotischer Pseudointima zur Folge, wie sie vor allem in den Grafts mit porcinem Gewebe zu beobachten war.

Fast alle untersuchten Conduits wiesen degenerative Veränderungen in Form von Kalkablagerungen auf. Es zeigte sich eine Korrelation zwischen Implantationszeit und Ausmaß der Kalzifizierung. Im Vergleich der unterschiedlichen Conduit-Typen war das porcine Gewebe der Hancock-Conduits am stärksten betroffen. Da in diesen Explantaten auch die inflammatorischen Reaktionen besonders ausgeprägt waren, kann ein Zusammenhang zwischen Entzündungsaktivität und Degeneration angenommen werden.

Zusammenfassend lässt sich feststellen, dass Conduit-Implantationen in den rechtsventrikulären Ausflusstrakt zu einer Gewebereaktion führen, die in den drei analysierten Grafttypen grundlegende Gemeinsamkeiten aufweist. Jedoch zeigen sich in Xenografts eine deutlicher ausgeprägte Entzündungsreaktion und in der Folge ausgedehntere degenerative Veränderungen als in Homografts. So dürfte dieser Befund eine wichtige Grundlage sein für die klinisch zu beobachtende Überlegenheit von Homografts in Bezug auf die Dauer des Funktionserhaltes.

Möglicherweise lassen sich aus den Ergebnissen zur Biokompatibilität von RVOT-Conduits für die Zukunft prophylaktische und therapeutische Ansätze ableiten, durch die die Funktion von kardiovaskulären Implantaten weiter verbessert werden kann. 


\title{
Kapitel 6
}

\section{Abkürzungs- und Abbildungsverzeichnis}

\author{
BAP Ballonangioplastie \\ BVP Ballonvalvuloplastie \\ CD Cluster of Differentiation \\ DAB Diaminobenzidin \\ EDTA Ethylendiamintetraessigsäure \\ EvG Elastica-van-Giesson-Färbung \\ EZM Extrazellulärmatrix \\ HE Hämatoxylin-Eosin-Färbung \\ PTFE Polytetrafluorethylen \\ RVOT rechtsventrikulärer Ausflusstrakt \\ SMA Smooth Muscle Actin \\ SMMy Smooth Muscle Myosin \\ TAC Truncus arteriosus communis \\ TGA Transposition der großen Arterien \\ TOF Fallot'sche Tetralogie \\ VSD Ventrikelseptumdefekt
}




\title{
Abbildungsverzeichnis
}

\author{
1.1 A Contegra(R)-Conduit, Quelle: Medtronic Inc.; B Hancock $($ )- \\ Conduit, Quelle: Medtronic Inc. . . . . . . . . . . . . . 5
}

$3.1 \quad$ A positive Färbung (braun) für Kollagen-III-Fasern in Intima

(I) und Media (M); Vergrößerung 200fach; Kontroll-Homograft; B von-Willebrand-Faktor-positive Zellen lumenseitig auf der Intima sowie in kleinen Gefäßen der Adventitia; Vergrößerung 100fach; Kontroll-Homograft . . . . . . . . . . . . . . 15

3.2 stark gewellte elastische Fasern der Media; EvG; Vergrößerung 400fach; Kontroll-Homograft . . . . . . . . . . . . . . . . . 15

3.3 A SMMy-positive fibromuskuläre Zellen der Pseudointima; Vergrößerung 400fach; Präparat 6; B Desmin-positive fibromuskuläre Zellen der Pseudointima; Vergrößerung 200fach; Präparat 216

3.4 lumenseitig Vimentin-positive Endothelzellen; Vergrößerung 400fach; Präparat 4........................ 16

3.5 A septierte Pseudointima; Färbung nach Richardson; Vergrößerung 200fach; Präparat 8; B Entzündungszellen in den Fibrinfäden der Pseudointima; Fibrinfärbung Vergrößerung 400fach; Präpa-

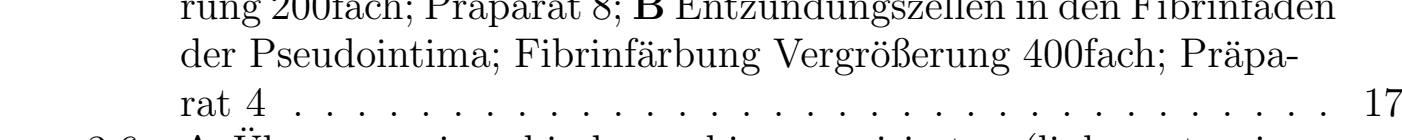

3.6 A Übergang eines bindegewebig organisierten (links unten im Bild) zu einem septierten Abschnitt der Pseudointima; Färbung nach Richardson; Vergrößerung 200fach; Präparat 4; B septierte Pseudointima im Klappen-Winkel; Färbung nach Richardson; Vergrößerung 200fach; Präparat 4 . . . . . . . . . . . . . 17

3.7 septierte Pseudointima an der Klappenbasis; Färbung nach Richardson; Vergrößerung 400fach; Präparat 3 . . . . . . . . . . 17

3.8 verzweigte Lakunen in der Pseudointima; von-Willebrand-Färbung;

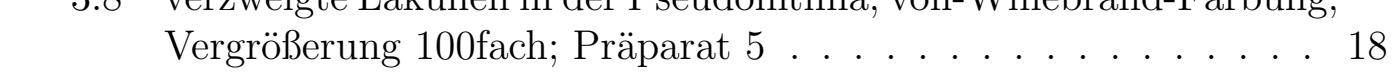

3.9 porzellanartige Verkalkung; anterograde Durchleuchtung des Präparats; Präparat 6 . . . . . . . . . . . . . . . . 19

3.10 Kalkareal in der Pseudointima; Färbung nach Richardson; Ver-

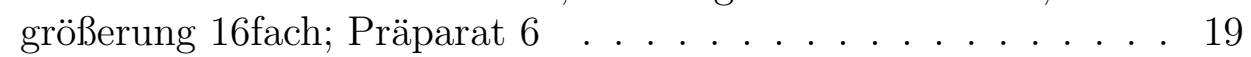

3.11 intra- und extrazelluläre Verkalkung (schwarz) in der Pseudointima; Färbung nach von Kossa; Vergrößerung 400fach; Präparat $5 \ldots \ldots \ldots \ldots \ldots$ 
3.12 A Klappensegel (KS) an dem Kalkareal in der Pseudointima; Färbung nach Richardson; Vergrößerung 16fach; Präparat 1; B Entzündungszellen und Erythrozyten innerhalb des Kalkareals am Klappensegel; Ausschnitt aus A; Färbung nach Richardson; Vergrößerung 100fach; Präparat 1 . . . . . . . . . . . . 20

3.13 A chondroide Metaplasie in der Pseudointima; Färbung nach Richardson; Vergrößerung 400fach; Präparat 1; B chondroide Metaplasie in der Pseudointima; Färbung nach Richardson; Vergrößerung 200fach; Präparat 2 . . . . . . . . . . . 20

3.14 CD3-positive T-Zellen in der septierten Pseudointima in den Falten des Conduits; Vergrößerung 400fach; Präparat 4 . . . . . 21

3.15 A Kollagenfasern der Subtypen I (orange) und III (grün) im Homograft-Gewebe; Pikrosirius-Rot-Färbung; Vergrößerung 200fach; Präparat 5; B SMA-positive fibromuskuläre Zellen im HomograftGewebe; Vergrößerung 400fach; Präparat 3 . . . . . . . . . . 22

3.16 Entzündungszellen um Nahtmaterial (N) im Homograft-Gewebe; Färbung nach Richardson; Vergrößerung 100fach; Präparat 8 . . 22

3.17 A CD8-positive T-Killerzellen zwischen den Fasern des HomograftGewebes; Vergrößerung 400fach; Präparat 4; B Infiltrat CD79positiver B-Lymphozyten im Graft-Gewebe; Vergrößerung 200fach;

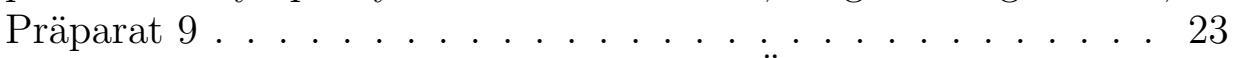

3.18 A Fettzellen in der Adventitia (nach rechts Übergang zum HomograftGewebe); Färbung nach Richardson; Vergrößerung 40fach; Präparat 3; B Vimentin-positive Zellen in Muskelfaser-Bündeln der Adventitia; Vergrößerung 100fach; Präparat 2 . . . . . . . . 24

3.19 SMA-positive Zellen der Vasa vasorum in der Adventitia; Vergrößerung 100fach; Präparat 9 . . . . . . . . . . . . 24

3.20 Ansammlung CD3-positiver T-Lymphozyten in der Adventitia; Vergrößerung 100fach; Präparat 2 . . . . . . . . . . . . . . 24

3.21 CD68-positive FKRZ um gewobene Fäden in der Adventitia; Vergrößerung 400fach; Präparat 2 . . . . . . . . . . . 25

3.22 B-Zell-Infiltrat im Homograft-Gewebe an der Grenze zur Adven-

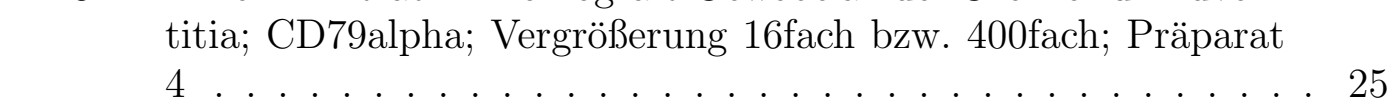

3.23 Plasmazellen im Homograft-Gewebe am Übergang zur Adventitia; CD138; Präparat 4 . . . . . . . . . . . . . 26

3.24 A Ansatz zweier Klappensegel (KS) an der Conduit-Wand; Färbung nach Richardson; Vergrößerung 16fach; Präparat 4; B zahlreiche Zellen im Pseudointima-Gewebe der Klappe, während das Graft-Gewebe der Klappe zellarm ist; Färbung nach Richardson; Vergrößerung 400fach; Präparat 1 . . . . . . . . . . . 26

3.25 A septiertes Pseudointima-Gewebe an der Klappe; Färbung nach Richardson; Vergrößerung 400fach; Präparat 3; B PseudointimaGewebe an der Klappe mit Kapillaren und extravasalen ErythrozytenAnsammlungen; Färbung nach Richardson; Vergrößerung 40fach;

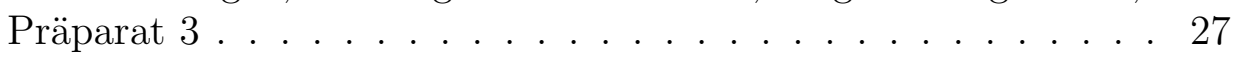

3.26 Einblutungen an den Klappensegeln; Präparat 3 . . . . . . . . . 27 
3.27 A verkalktes Klappensegel und Kalkherd im Klappensinus; Färbung nach Richardson; Vergrößerung 16fach; Präparat 10; B Präparat 10 nach Zuschnitt . . . . . . . . . . . . . . . . . . . . . . . 27

3.28 CD68-positive Histiozyten in der Klappe; Vergrößerung 40fach bzw. ca 200fach; Präparat 3 . . . . . . . . . . . . . . . . 28

3.29 makroskopisch unauffällige Oberfläche der Pseudointima mit überwachsenen Stentstreben; Präparat 8 . . . . . . . . . . . . 28

3.30 A unterschiedliche Bereiche der Pseudointima (links im Bild Lumen); Färbung nach Richardson; Vergrößerung 100fach; Präparat 9; B SMA-positive Zellen im lumenseitig (links im Bild) gelegenen Bereich der Pseudointima; Vergrößerung 200fach; Präpa-

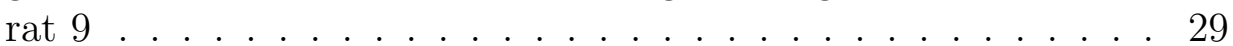

3.31 Histiozyten und Fremdkörperriesenzellen; Metallstreben (M) des Stents teilweise herausgelöst (nach links Lumen); CD68; Ver-

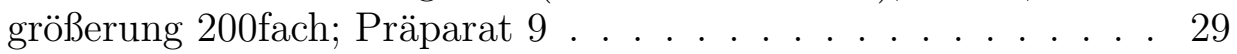

3.32 A Kollagen I (orange) in der Pseudointima um die Stent-Streben; Pikrosirius-Rot-Färbung; Vergrößerung 200fach; Präparat 8; B Vimentin-positive fibromuskuläre Zellen in der Pseudointima um den Stent; Vergrößerung 200fach; Präparat 8 . . . . . . . . . 30

3.33 Verkalkungsarelae in der Pseudointima (unten Lumen); Färbung nach Richardson; Vergrößerung 100fach; Präparat 7 . . . . . . . 30

3.34 A Präparat 13 nach Zuschnitt; B Kollagen-III-Fasern in der Pseudointima (unten links Lumen); Pikrosirius-Rot-Färbung; Vergrößerung 200fach; Präparat 13 $\ldots \ldots \ldots \ldots . \ldots \ldots$

3.35 CD138-positive Plasmazellen perivaskulär in der Pseudointima; Vergrößerung 400fach; Präparat 13 . . . . . . . . . . . . . 32

3.36 CD3-positive T-Lymphozyten in der Pseudointima zwischen den Klappenabgängen; Vergrößerung 400fach; Präparat 11. . . . . . 32

3.37 A makroskopisch erkennbarer wandständiger Thrombus im Präparat 11 nach Zuschnitt; Präparat 11; $\mathbf{B}$ wandständiger Thrombus; Fibrinfärbung; Vergrößerung 16fach . . . . . . . . . . . . . 33

3.38 Kalkeinschlüsse im Thrombus; Färbung nach von Kossa; Vergrößerung 16fach; Präparat 11 . . . . . . . . . . . . . 33

3.39 wandständiger Thrombus mit organisierten und amorphen Bereichen sowie Entzündungszellen; HE; Vergrößerung 16fach bzw. 40fach; Präparat 11. . . . . . . . . . . . . . . 34

3.40 A Makrophagen in der amorphen Substanz des Thrombus; CD68; Vergrößerung 200fach; Präparat 11; B Endothel lumenseitig an der Pseudointima und in den Kapillaren (unten Lumen); vonWillebrand-Färbung; Vergrößerung 100fach; Präparat 13 $\ldots$. . . . 34

3.41 Schichtung der Wand, im Bild rechts (unten rechts Lumen) dichte gewellte Fasern der Pseudointima, angrenzend bindegewebiges Graft-Gewebe; HE; Vergrößerung 40fach; Präparat 11 . . . . 35 
3.42 A Vimentin-positive Zellen im Graft-Gewebe; rechts im Bild gerade Fasern, links gewellte Fasern; Vergrößerung 200fach; Präparat 13; B SMA-positive fibromuskuläre Zellen und Fasern unterschiedlicher Struktur im Graft-Gewebe (links im Bild zum Lumen weisender Anteil des Gewebes); Vergrößerung 400fach; Präparat 13 . . . . . . . . . . . . . . . . . . . 35

3.43 A Fremdkörperriesenzellen und Histiozyten zwischen den stark gewellten Fasern des Grafts; nur wenige, kleine Histiozyten in der Schicht mit weniger stark gewellten Fasern; CD68; Vergrößerung 400fach; Präparat 11; B gruppierte B-Lymphozyten im gewellten Graft-Bereich in Klappen-Nähe; CD79alpha; Vergrößerung 400fach; Präparat 11 . . . . . . . . . . . . . 36

3.44 A feine elastische Fasern um die Kapillaren der Adventitia; EvG; Vergrößerung 200fach; Präparat 12; B Kapillaren in der Adventitia; SMA; Vergrößerung 100fach; Präparat 11 . . . . . . 37

3.45 h-Caldesmon-positive Zellen in den Kapillarwänden der Adventitia; Vergrößerung 100fach; Präparat 12 . . . . . . . . . . . 37

3.46 Lymphozyten-Infiltrat in der Adventitia; HE; Vergrößerung 400fach; Präparat 13 . . . . . . . . . . . . . . . 37

3.47 zahlreiche Kapillaren und Lymphozyten in der Adventitia; Vimentin; Vergrößerung 200fach; Präparat 12 . . . . . . . . . . . . 38

3.48 A Kollagenfasern im erhaltenen Klappensegel; Pikrosirius-RotFärbung; Vergrößerung 100fach; Präparat 11; B CD68-positive Fremdkörperriesenzellen im Klappensegel; Vergrößerung 400fach; Präparat 11 . . . . . . . . . . . . . . . . . . . . . 39

3.49 Kalkherde im Klappensegel; Färbung nach von Kossa; Vergrößerung 100fach; Präparat 11 . . . . . . . . . . . . . . . . . . . 39

3.50 A fibröses Klappengerüst in der Conduit-Wand und Klappenursprung; HE; Vergrößerung 16fach; Präparat 11; B elastische Fasern im Klappengerüst; lumenseitig amorphe Substanz der thrombotischen Pseudointima zwischen den Klappenursprüngen; EvG; Vergrößerung 400fach; Präparat 11 . . . . . . . . . . . . . 40

3.51 kurze elastische Fasern in den Klappensegeln; EvG; Vergrößerung 100fach; Präparat 12 . . . . . . . . . . . . . . 40

3.52 A verdickte Pseudointima außerhalb der Klappenebene; Präparat 24 nach Zuschnitt; B Pseudointima; SMA; Vergrößerung 200fach; Präparat $23 \ldots \ldots \ldots \ldots$. . . . . . . . . . 41

3.53 A Pseudointima und PTFE; HE; Vergrößerung 100fach; Präparat 15; B Pseudointima und PTFE; SMA; Vergrößerung 200fach;

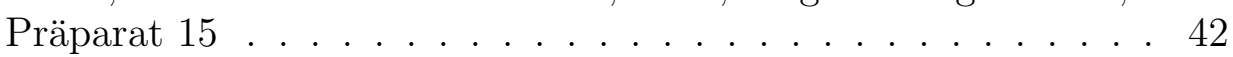

3.54 A septierte Pseudointima; Färbung nach Richardson; Vergrößerung 400fach; Präparat 17; B thrombotische Abschnitte der Pseudointima; Färbung nach Richardson; Vergrößerung 100fach; Präparat 18 . . . . . . . . . . . . . . . . . . 42 
3.55 A septierte Pseudointima im Winkel zwischen Klappe und ConduitWand; Färbung nach Richardson; Vergrößerung 16fach; Präparat 23; B Vergrößerung aus A: Entzündungszellen und Erythrozyten zwischen den Septen; Färbung nach Richardson; Vergrößerung 200fach; Präparat 23 . . . . . . . . . . . . . . . . . . 43

3.56 A thrombotisches Pseudointima-Gewebe nahe der PTFE-Membran; Färbung nach Richardson; Vergrößerung 40fach; Präparat 19; B fibrinreiche thrombotische Pseudointima; Fibrinfärbung; Vergrößerung 40fach; Präparat 15 . . . . . . . . . . . . . 43

3.57 A lumenseitiges Endothel; von-Willebrand-Färbung; Vergrößerung 400fach; Präparat 14; B Kapillaren im zum Graft gewandten Teil der Pseudointima (Gewebe präparationsbedingt im Bereich des PTFE ausgerissen); SMA; Vergrößerung 200fach; Präparat 14 . . . . . . . . . . . . . . . . . . . 44

3.58 A typischer makroskopischer Aspekt flächiger Verkalkung; Präparat 15; $\mathbf{B}$ mikroskopischer Befund deutlicher, schichtübergreifender Verkalkung (*); Färbung nach Richardson; Vergrößerung 16fach; Präparat 15 . . . . . . . . . . . . . . . . 44

3.59 A ausgedehnte Lymphozyten-Infiltrate in PTFE-Nähe in der Pseudointima; Zellen positiv für CD79 (siehe Bild) sowie CD3 und CD8; CD79; Vergrößerung 100fach; Präparat 21; B B-Zellen aus A; CD79; Vergrößerung 400fach; Präparat 21 . . . . . . . . 45

3.60 T-Lymphozyten perivaskulär in der Pseudointima; CD3; Vergrößerung 200fach; Präparat 18 $\ldots \ldots \ldots \ldots \ldots$

3.61 A Entzündungszellen und Erythrozyten in der Pseudointima (unten Lumen); Färbung nach Richardson; Vergrößerung 400fach; Präparat 14; B makroskopisch infiziert wirkendes thrombotisches Material an einem der Klappensegel; Färbung nach Richardson; Vergrößerung 40fach; Präparat 14 . . . . . . . . . . 46

3.62 weiter proximal weniger Entzündungszellen in der septierten Pseudointima; HE; Vergrößerung 200fach; Präparat 14 . . . . . 46

3.63 A Graft-Gewebe eines Hancock-Conduits aus PTFE-Material und dichtem Bindegewebe (BG) des porcinen klappentragenden Segments; links im Bild Anteile der Pseudointima (PI); Färbung nach Richardson; Vergrößerung 200fach; Präparat 14; B flächige Verkalkung im porcinen Gewebe (unten Lumen); Färbung nach Richardson; Vergrößerung 40fach; Präparat 17 $\ldots$. . . . . . . . 47

3.64 Verkalkung auch im PTFE-Gewebe; Färbung nach Richardson; Vergrößerung 16fach; Präparat 15 . . . . . . . . . . . . . . . 47

3.65 A einzeln liegende CD68-positive Makrophagen ausschließlich im Randbereich des Schweine-Gewebes; Vergrößerung 400fach; Präparat 23; B CD68-positive Fremdkörperriesenzellen im Randbereich des porcinen Gewebes (porc.) angrenzend an die Pseudointima (PI); Vergrößerung 200fach; Präparat 23 $\ldots \ldots$. . . . . . 48

3.66 CD68-positive Histiozyten und mehrkernige Riesenzellen um die PTFE-Fasern; Vergrößerung 200fach; Präparat 20 . . . . . . . . 48 
3.67 A CD8-positive T-Killerzellen im Adventitia-Gewebe zwischen PTFE-Membran und Metallring; Vergrößerung 200fach; Präparat 18; B CD8-positive T-Killerzellen, Ausschnitt aus A; Ver-

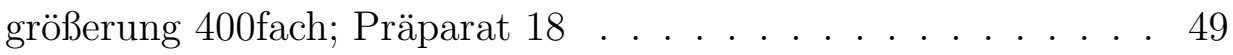

3.68 typischer Aspekt der Adventitia; SMA; Vergrößerung 40fach; Präparat 15 . . . . . . . . . . . . . . . . . . 49

3.69 A Schweine-Gewebe des Klappensegels mit thrombotischer Pseudointima; Färbung nach Richardson; Vergrößerung 100fach; Präparat 16; B durch Pseudointima (PI) überdecktes Klappensegel (KS); Färbung nach Richardson; Vergrößerung 16fach; Präpa-

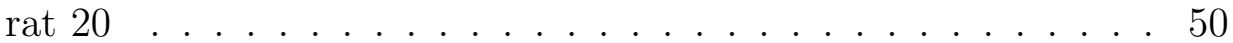

3.70 A Präparat 21 nach Zuschnitt; B ein Teil des Klappensegels bedeckt und an der Pseudointima im Klappensinus haftend; Färbung nach Richardson; Vergrößerung 16fach; Präparat 21] . . 51

3.71 A ein Klappensegel makroskopisch durch Pseudointima verdeckt; Präparat 23; B Pseudointima-Brücke (PI) zwischen ConduitWand und Klappensegel (KS); Färbung nach Richardson; Vergrößerung 16fach; Präparat 23 . . . . . . . . . . . 51

3.72 von-Willebrand-Faktor-positive Endothelschicht auf der Klappe; Vergrößerung 200fach; Präparat 23 . . . . . . . . . . . 52

3.73 A Pseudointima-Gewebe im Klappensinus mit Kapillaren; vonWillebrand-Färbung; Vergrößerung 16fach; Präparat 20; B Pseudointima-Gewebe im Klappensinus mit Kapillaren, vergrößerter Ausschnitt aus Abbildung A; von-Willebrand-Färbung; Vergrößerung 100fach; Präparat 20 . . . . . . . . . . . . . . . 52

3.74 verkalktes Segel der insuffizienten Klappe; Färbung nach Richardson; Vergrößerung 16fach; Präparat 18 . . . . . . . . . 52

3.75 A Präparat 17 nach Zuschnitt; $\mathbf{B}$ verkalkte Klappenrudimente; Färbung nach Richardson; Vergrößerung 16fach; Präparat 17 . . 53 


\section{Kapitel 7}

\section{Literaturverzeichnis}

Agarwal KC, Edwards WD, Feldt RH, Danielson GK, Puga FJ, McGoon DC (1981): Clinicopathological correlates of obstructed right-sided porcine-valved extracardiac conduits. J Thorac Cardiovasc Surg 81:591-601.

Agarwal KC, Edwards WD, Feldt RH, Danielson GK, Puga FJ, McGoon DC (1982): Pathogenesis of nonobstructive fibrous peels in rightsided porcine-valved extracardiac conduits. J Thorac Cardiovasc Surg 83:584-589.

Aikawa M, Sakomura Y, Ueda M, Kimura K, Manabe I, Ishiwata S, Komiyama N, Yamaguchi H, Yazaki Y, Nagai R (1997): Redifferentiation of smooth muscle cells after coronary angioplasty determined via myosin heavy chain expression. Circulation 96:82-90.

Anderson JM, Miller KM (1984): Biomaterial biocompatibility and the macrophage. Biomaterials $\underline{5}: 5-10$.

Anderson JM, Rodriguez A, Chang DT (2008): Foreign body reaction to biomaterials. Semin Immunol 20:86-100.

Ando M, Tomizawa Y, Noishiki Y, Terada M, Imai Y (2000): Autologous tissue-fragmented extracardiac conduit with rapid, stable endothelialization due to angiogenesis. Jpn J Thorac Cardiovasc Surg 48:153-160.

Armiger LC (1995): Viability studies of human valves prepared for use as allografts. Ann Thorac Surg $\underline{60}$ :S118-21.

Bando K, Danielson GK, Schaff HV, Mair DD, Julsrud PR, Puga FJ (1995): Outcome of pulmonary and aortic homografts for right ventricular outflow tract reconstruction. J Thorac Cardiovasc Surg 109:509-17; discussion 517-8.

Baskett RJ, Ross DB, Nanton MA, Murphy DA (1996): Factors in the early failure of cryopreserved homograft pulmonary valves in children: preserved immunogenicity? J Thorac Cardiovasc Surg 112:1170-8; discussion $1178-9$.

Berger K, Sauvage LR, Rao AM, Wood SJ (1972): Healing of arterial prostheses in man: its incompleteness. Ann Surg 175:118-127. 
Boethig D, Thies WR, Hecker H, Breymann T (2005): Mid term course after pediatric right ventricular outflow tract reconstruction: a comparison of homografts, porcine xenografts and Contegras. Eur J Cardiothorac Surg 27:58-66.

Bonfield TL, Colton E, Anderson JM (1992): Protein adsorption of biomedical polymers influences activated monocytes to produce fibroblast stimulating factors. J Biomed Mater Res 26:457-465.

Bottio T, Thiene G, Pettenazzo E, Ius P, Bortolotti U, Rizzoli G, Valfré C, Casarotto D, Valente M (2003): Hancock II bioprosthesis: a glance at the microscope in mid-long-term explants. J Thorac Cardiovasc Surg 126:99-105.

Boudjemline Y, Bonnet D, Massih TA, Agnoletti G, Iserin F, Jaubert F, Sidi D, Vouhé P (2003): Use of bovine jugular vein to reconstruct the right ventricular outflow tract: early results. J Thorac Cardiovasc Surg 126:490-497.

Breymann T, Boethig D, Goerg R, Thies WR (2004): The Contegra bovine valved jugular vein conduit for pediatric RVOT reconstruction: 4 years experience with 108 patients. J Card Surg 19:426-431.

Brockbank KG, Song YC, Khirabadi BS, Lightfoot FG, Boggs JM, Taylor MJ (2000): Storage of tissues by vitrification. Transplant Proc 32:3-4.

Brown JW, Ruzmetov M, Rodefeld MD, Vijay P, Turrentine MW (2005): Right ventricular outflow tract reconstruction with an allograft conduit in non-ross patients: risk factors for allograft dysfunction and failure. Ann Thorac Surg 80:655-63; discussion 663-4.

Brown JW, Ruzmetov M, Rodefeld MD, Vijay P, Darragh RK (2006): Valved bovine jugular vein conduits for right ventricular outflow tract reconstruction in children: an attractive alternative to pulmonary homograft. Ann Thorac Surg 82:909-916.

Butany J, Collins MJ (2005): Analysis of prosthetic cardiac devices: a guide for the practising pathologist. J Clin Pathol 58:113-124.

Butany J, Yu W, Silver MD, David TE (1999): Morphologic findings in explanted Hancock II porcine bioprostheses. J Heart Valve Dis ㅇ:4-15.

Butany J, Ahluwalia MS, Nair V, David TE (2004): Cryopreserved pulmonary homograft: postimplant changes. Cardiovasc Pathol 13:59-61.

Caldarone CA, McCrindle BW, Arsdell GSV, Coles JG, Webb G, Freedom RM, Williams WG (2000): Independent factors associated with longevity of prosthetic pulmonary valves and valved conduits. J Thorac Cardiovasc Surg 120:1022-31.

Chang Y, Tsai CC, Liang HC, Sung HW (2001): Reconstruction of the right ventricular outflow tract with a bovine jugular vein graft fixed with a naturally occurring crosslinking agent (genipin) in a canine model. $J$ Thorac Cardiovasc Surg 122:1208-1218. 
Cleveland DC, Williams WG, Razzouk AJ, Trusler GA, Rebeyka IM, Duffy L, Kan Z, Coles JG, Freedom RM (1992): Failure of cryopreserved homograft valved conduits in the pulmonary circulation. Circulation 86:II150-II153.

Collett GDM, Canfield AE (2005): Angiogenesis and pericytes in the initiation of ectopic calcification. Circ Res 96:930-938.

Corno AF, Hurni M, Griffin H, Galal OM, Payot M, Sekarski N, Tozzi P, von Segesser LK (2002): Bovine jugular vein as right ventricletopulmonary artery valved conduit. J Heart Valve Dis 11:242-8.

Corno AF, Qanadli SD, Sekarski N, Artemisia S, Hurni M, Tozzi P, von Segesser LK (2004): Bovine valved xenograft in pulmonary position: medium-term follow-up with excellent hemodynamics and freedom from calcification. Ann Thorac Surg $\underline{78: 1382-8 .}$

Dave HH, Kadner A, Berger F, Seifert B, Dodge-Khatami A, Bettex D, Pretre R (2005): Early results of the bovine jugular vein graft used for reconstruction of the right ventricular outflow tract. Ann Thorac Surg 79:618-624.

Dìaz-Flores L, Valladares F, Gutierrez R, Varela H (1990): The role of the pericytes of the adventitial microcirculation in the arterial intimal thickening. Histol Histopathol $\underline{5}: 145-153$.

Dearani JA, Danielson GK, Puga FJ, Schaff HV, Warnes CW, Driscoll DJ, Schleck CD, Ilstrup DM (2003): Late follow-up of 1095 patients undergoing operation for complex congenital heart disease utilizing pulmonary ventricle to pulmonary artery conduits. Ann Thorac Surg 75:399-411.

Doherty MJ, Ashton BA, Walsh S, Beresford JN, Grant ME, Canfield AE (1998): Vascular pericytes express osteogenic potential in vitro and in vivo. J Bone Miner Res 13:828-838.

Dong ZMD, Aplin AC, Nicosia RF (2009): Regulation of angiogenesis by macrophages, dendritic cells, and circulating myelomonocytic cells. Curr Pharm Des 15:365-379.

Edwards WD, Agarwal KC, Feldt RH, Danielson GK, Puga FJ (1983): Surgical pathology of obstructed, right-sided, porcine-valved extracardiac conduits. Arch Pathol Lab Med 107:400-405.

Ehler WJ, Cissik JH, Smith VC, Hubbard GB (1990): Evaluation of GoreTex graft material in the repair of right ventricular outflow tract defect. J Invest Surg 3:119-127.

Erez E, Sandbank J, Stamler A, Raanani E, Sharoni E, Vidne BA, Barak J (2001): Structural modification and cryopreservation of porcine heart valves for xenotransplantation with reduced immunity. $J$ Heart Valve Dis 10:125-128. 
Farb A,Weber DK, Kolodgie FD, Burke AP, Virmani R (2002): Morphological predictors of restenosis after coronary stenting in humans. Circulation 105:2974-2980.

Farrington-Rock C, Crofts NJ, Doherty MJ, Ashton BA, Griffin-Jones C, Canfield AE (2004): Chondrogenic and adipogenic potential of microvascular pericytes. Circulation 110:2226-2232.

Fischlein T, Schütz A, Haushofer M, Frey R, Uhlig A, Detter C, Reichart B (1995): Immunologic reaction and viability of cryopreserved homografts. Ann Thorac Surg 60:S122-6.

Foth R, Quentin T, Michel-Behnke I, Vogt M, Kriebel T, Kreischer A, Ruschewski W, Paul T, Sigler M (2009): Immunohistochemical characterization of neotissues and tissue reaction to septal defect occlusion devices. Circ Cardiovasc Interv 2:90-96.

Frazier OH, Baldwin RT, Eskin SG, Duncan JM (1993): Immunochemical identification of human endothelial cells on the lining of a ventricular assist device. Tex Heart Inst $J$ 20:78-82.

Frid MG, Dempsey EC, Durmowicz AG, Stenmark KR (1997): Smooth muscle cell heterogeneity in pulmonary and systemic vessels. Importance in vascular disease. Arterioscler Thromb Vasc Biol 17:1203-1209.

Göber V, Berdat P, Pavlovic M, Pfammatter JP, Carrel TP (2005): Adverse mid-term outcome following RVOT reconstruction using the Contegra valved bovine jugular vein. Ann Thorac Surg 79:625-631.

Goffin M (1997): Morphologic study of homograft valves before and after cryopreservation and after short-term implantation in patients. Cardiovasc Pathol $\underline{6}: 35-41$.

Hayabuchi Y, Mori K, Kitagawa T, Sakata M, Kagami S (2007): Polytetrafluoroethylene graft calcification in patients with surgically repaired congenital heart disease: evaluation using multidetector-row computed tomography. Am Heart J 153:806-808.

Homann M, Haehnel JC, Mendler N, Paek SU, Holper K, Meisner H, Lange R (2000): Reconstruction of the RVOT with valved biological conduits: 25 years experience with allografts and xenografts. Eur $J$ Cardiothorac Surg 17:624-630.

Houel R, Moczar M, Clerin V, Loisance DY (1999): Pseudointima in inflow conduits of left ventricular assist devices. Ann Thorac Surg 68:717-723.

Hu Y, Davison F, Zhang Z, Xu Q (2003): Endothelial replacement and angiogenesis in arteriosclerotic lesions of allografts are contributed by circulating progenitor cells. Circulation 108:3122-3127.

Ichikawa Y, Noishiki Y, Kosuge T, Yamamoto K, Kondo J, Matsumoto A (1997): Use of a bovine jugular vein graft with natural valve for right ventricular outflow tract reconstruction: a one-year animal study. J Thorac Cardiovasc Surg 114:224-233. 
Ishizaka T, Ohye RG, Goldberg CS, Ramsburg SR, Suzuki T, Devaney EJ, Bove EL (2003): Premature failure of small-sized Shelhigh No- React porcine pulmonic valve conduit model NR-4000. Eur J Cardiothorac Surg 23:715-718.

Jashari R, Herijgers P, Verbeken E, A VL, Nishimura Y, Ràcz R, Flameng W (2001): Calcification and degeneration characteristics of the Biocor noreact bovine internal mammary artery (BIMA) - in vivo evaluation in a sheep model. Cardiovasc Surg 9:44-49.

Javadpour H, Veerasingam D, Wood AE (2002): Calcification of homograft valves in the pulmonary circulation - is it device or donation related? Eur J Cardiothorac Surg 22:78-81.

Jeong UG, Khan AA, Fine G, Folger GM, Gonzalez-Lavin L (1976): Degenerative changes in aortic root allografts placed in the right ventricular outflow tract of growing puppies. Thorax 31:757-764.

Jeziorska M, Woolley DE (1999): Local neovascularization and cellular composition within vulnerable regions of atherosclerotic plaques of human carotid arteries. J Pathol 188:189-196.

Kim WH, Min SK, Choi CH, Lee JR, Kim YJ, Bae EJ, Noh CI (2007): Follow-up of Shelhigh porcine pulmonic valve conduits. Ann Thorac Surg 84:2047-2050.

Koolbergen DR, Hazekamp MG, de Heer E, van Hoorn F, Huysmans HA, Bruijn JA, Dion RAE (2002a): Structural degeneration of pulmonary homografts used as aortic valve substitute underlines early graft failure. Eur J Cardiothorac Surg 22:802-807.

Koolbergen DR, Hazekamp MG, de Heer E, Bruggemans EF, Huysmans HA, Dion RAE, Bruijn JA (2002b): The pathology of fresh and cryopreserved homograft heart valves: an analysis of forty explanted homograft valves. J Thorac Cardiovasc Surg 124:689-697.

Kreischer A: Biokompatibilitätsuntersuchung an Implantaten zur interventionellen Therapie von angeborenen Herzfehlern. Med. Diss. Göttingen 2008 .

Lange R, Weipert J, Homann M, Mendler N, Paek SU, Holper K, Meisner H (2001): Performance of allografts and xenografts for right ventricular outflow tract reconstruction. Ann Thorac Surg 1:365-367.

Legare JF, Lee TD, Creaser K, Ross DB (2000): T lymphocytes mediate leaflet destruction and allograft aortic valve failure in rats. Ann Thorac Surg 70:1238-1245.

Lillehei CW, Varco RL, Cohen M, Warden HE, Gott VL, DeWall RA, Patton C, Moller JH (1986) The first open heart corrections of tetralogy of Fallot. A 26-31 year follow-up of 106 patients. Ann Surg 204:490-502. 
Lin PH, Chen C, Bush RL, Yao Q, Lumsden AB, Hanson SR (2004): Small-caliber heparin-coated ePTFE grafts reduce platelet deposition and neointimal hyperplasia in a baboon model. J Vasc Surg 39:1322-1328.

Livi U, Abdulla AK, Parker R, Olsen EJ, Ross DN (1987): Viability and morphology of aortic and pulmonary homografts. A comparative study. $J$ Thorac Cardiovasc Surg 93:755-760.

Lupinetti FM, Tsai TT, Kneebone JM, Bove EL (1993): Effect of cryopreservation on the presence of endothelial cells on human valve allografts. J Thorac Cardiovasc Surg 106:912-917.

Mair DD, Fulton RE, Danielson GK (1978): Thrombotic occlusion of Hancock conduit due to severe dehydration after Fontan operation. Mayo Clin Proc 53:397-402.

Manji RA, Zhu LF, Nijjar NK, Rayner DC, Korbutt GS, Churchill TA, Rajotte RV, Koshal A, Ross DB (2006): Glutaraldehyde-fixed bioprosthetic heart valve conduits calcify and fail from xenograft rejection. Circulation 114:318-327.

Mathieu P, Roussel JC, Dagenais F, Anegon I (2003): Cartilaginous metaplasia and calcification in aortic allograft is associated with transforming growth factor beta 1 expression. J Thorac Cardiovasc Surg 126:1449-1454.

Meyns B, Garsse LV, Boshoff D, Eyskens B, Mertens L, Gewillig M, Fieuws S, Verbeken E, Daenen W (2004): The Contegra conduit in the right ventricular outflow tract induces supravalvular stenosis. J Thorac Cardiovasc Surg 128:834-840.

Milano A, Bortolotti U, Talenti E, Valfré C, Arbustini E, Valente M, Mazzucco A, Gallucci V, Thiene G (1984): Calcific degeneration as the main cause of porcine bioprosthetic valve failure. Am J Cardiol 53:1066-1070.

Mitchell RN, Jonas RA, Schoen FJ (1995): Structurefunction correlations in cryopreserved allograft cardiac valves. Ann Thorac Surg 60:S108-12; Diskussion S113.

Mitchell RN, Jonas RA, Schoen FJ (1998): Pathology of explanted cryopreserved allograft heart valves: comparison with aortic valves from orthotopic heart transplants. J Thorac Cardiovasc Surg 115:118-127.

Mohler ER, Gannon F, Reynolds C, Zimmerman R, Keane MG, Kaplan FS (2001): Bone formation and inflammation in cardiac valves. Circulation 103:1522-1528.

Molina JE, Edwards JE, Bianco RW, Clack RW, Lang G, Molina JR (1995): Composite and plain tubular synthetic graft conduits in right ventricle-pulmonary artery position: fate in growing lambs. J Thorac Cardiovasc Surg 110:427-435.

Moulton KS (2001): Plaque angiogenesis and atherosclerosis. Curr Atheroscler Rep 3:225-233. 
Müller KM, Dasbach G (1994) The pathology of vascular grafts. Curr Top Pathol 86:273-306.

Neves J, Monteiro C, Santos R, Martins A, Ramos S, Ramos T, Calta C, Rueff J, Melo JQ (1995): Histologic and genetic assessment of explanted allograft valves. Ann Thorac Surg 60:S141-S145.

Newby AC, Zaltsman AB (2000): Molecular mechanisms in intimal hyperplasia. J Pathol 190:300-309.

Ota T, Taketani S, Iwai S, Miyagawa S, Furuta M, Hara M, Uchimura E, Okita Y, Sawa Y (2007): Novel method of decellularization of porcine valves using polyethylene glycol and gamma irradiation. Ann Thorac Surg 83:1501-1507.

Park JC, Song MJ, Hwang YS, Suh H (2001): Calcification comparison

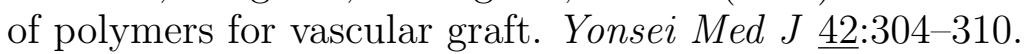

Pearl JM, Cooper DS, Bove KE, Manning PB (2002): Early failure of the Shelhigh pulmonary valve conduit in infants. Ann Thorac Surg $\underline{74}: 542-8$; discussion 548-9.

Pearson JD (1999): Endothelial cell function and thrombosis. Baillieres Best Pract Res Clin Haematol 12:329-341.

Perron J, Moran AM, Gauvreau K, del Nido PJ, Mayer JE, Jonas RA (1999): Valved homograft conduit repair of the right heart in early infancy. Ann Thorac Surg 68:542-548.

Qiao JH, Fishbein MC, Demer LL, Lusis AJ (1995): Genetic determination of cartilaginous metaplasia in mouse aorta. Arterioscler Thromb Vasc Biol 15:2265-2272.

Quentin T, Poppe A, Bär K, Sigler A, Foth R, Michel- Behnke I, Paul T, Sigler M (2009): A novel method for processing resinembedded specimens with metal implants for immunohistochemical labelling. Acta Histochem 111:538-542.

Raanani E, Yau TM, David TE, Dellgren G, Sonnenberg BD, Omran A (2000): Risk factors for late pulmonary homograft stenosis after the Ross procedure. Ann Thorac Surg 70:1953-1957.

Rafii S, Meeus S, Dias S, Hattori K, Heissig B, Shmelkov S, Rafii D, Lyden D (2002): Contribution of marrow-derived progenitors to vascular and cardiac regeneration. Semin Cell Dev Biol 13:61-67.

Rajamannan NM, Subramaniam M, Rickard D, Stock SR, Donovan J, Springett M, Orszulak T, Fullerton DA, Tajik AJ, Bonow RO, Spelsberg $\mathrm{T}$ (2003): Human aortic valve calcification is associated with an osteoblast phenotype. Circulation 107:2181-2184.

Rajani B, Mee RB, Ratliff NB (1998): Evidence for rejection of homograft cardiac valves in infants. J Thorac Cardiovasc Surg 115:111-117. 
Rastelli GC, Ongley PA, Davis GD, Kirklin JW (1965): Surgical Repair for Pulmonary Valve Atresia with coronary-pulmonaly artery Fistula: Report of Case. Mayo Clin Proc 40:521-527.

Rocchini AP, Weesner KM, Heidelberger K, Keren D, Behrendt D, Rosenthal A (1981): Porcine xenograft valve failure in children: an immunologic response. Circulation $\underline{64}$ :II162-II171.

Ross DN, Somerville J (1966): Correction of pulmonary atresia with a homograft aortic valve. Lancet 1966, 2:1446-1447.

Sanatani S, Potts JE, Human DG, Sandor GG, Patterson MW, Culham JAG (2001): Balloon angioplasty of right ventricular outflow tract conduits. Pediatr Cardiol 22:228-232.

Santini F, Piccin C, Prioli A, Pessotto R, Mazzucco A (1996): Accelerated aortic allograft fibrocalcification after right ventricular outflow tract reconstruction in pediatric patients: report of two cases. Eur J Cardiothorac Surg 10:290-293.

Sarkar S, Sales KM, Hamilton G, Seifalian AM (2007): Addressing thrombogenicity in vascular graft construction. J Biomed Mater Res B Appl Biomater 82:100-108.

Sartore S, Chiavegato A, Faggin E, Franch R, Puato M, Ausoni S, Pauletto P (2001): Contribution of adventitial fibroblasts to neointima formation and vascular remodeling: from innocent bystander to active participant. Circ Res 89:1111-1121.

Schmaltz A: Leitlinien zur Diagnostik und Therapie in der Pädiatrischen Kardiologie Urban\&Fischer, München 2007.

Schmaltz A, Bein G, Grävinghoff L, Hagel K, Hentrich F, Hofstetter R, Lindinger A, Kallfelz HC, Kramer HH, Mennicken U (1989): Balloon valvuloplasty of pulmonary stenosis in infants and children- cooperative study of the German Society of Pediatric Cardiology. Eur Heart $J \underline{10}: 967-971$.

Schoen FJ (1995): Approach to the analysis of cardiac valve prostheses as surgical pathology or autopsy specimens. Cardiovasc Pathol $\underline{4}: 241-255$.

Schreiber C, Sassen S, Kostolny M, Hörer J, Cleuziou J, Wottke M, Holper K, Fend F, Eicken A, Lange R (2006): Early graft failure of small-sized porcine valved conduits in reconstruction of the right ventricular outflow tract. Ann Thorac Surg 82:179-185.

Schulick AH, Taylor AJ, Zuo W, Qiu CB, Dong G, Woodward RN, Agah R, Roberts AB, Virmani R, Dichek DA (1998): Overexpression of transforming growth factor beta1 in arterial endothelium causes hyperplasia, apoptosis, and cartilaginous metaplasia. Proc Natl Acad Sci USA 95:6983-6988.

Scott SM, Barth MG, Gaddy LR, Ahl ET (1994): The role of circulating cells in the healing of vascular prostheses. J Vasc Surg 19:585-593. 
Shaddy RE, Hawkins JA (2002): Immunology and failure of valved allografts in children. Ann Thorac Surg $\underline{74}: 1271-1275$.

Shebani SO, McGuirk S, Baghai M, Stickley J, Giovanni JVD, Bulock FA, Barron DJ, Brawn WJ (2006): Right ventricular outflow tract reconstruction using Contegra valved conduit: natural history and conduit performance under pressure. Eur J Cardiothorac Surg 29:397-405.

Shi Q, Rafii S, Wu MH, Wijelath ES, Yu C, Ishida A, Fujita Y, Kothari S, Mohle R, Sauvage LR, Moore MA, Storb RF, Hammond WP (1998): Evidence for circulating bone marrow-derived endothelial cells. Blood 92:362-367.

Shi Y, O'Brien JE, Fard A, Mannion JD, Wang D, Zalewski A (1996): Adventitial myofibroblasts contribute to neointimal formation in injured porcine coronary arteries. Circulation 94:1655-1664.

Shimizu K, Sugiyama S, Aikawa M, Fukumoto Y, Rabkin E, Libby P, Mitchell RN (2001): Host bone-marrow cells are a source of donor intimal smooth- muscle-like cells in murine aortic transplant arteriopathy. Nat Med 7:738-741.

Sigler M, Jux C (2007): Biocompatibility of septal defect closure devices. Heart 93:444-449.

Sigler M, Handt S, Seghaye MC, von Bernuth G, Grabitz RG (2000): Evaluation of in vivo biocompatibility of different devices for interventional closure of the patent ductus arteriosus in an animal model. Heart 83:570-573.

Sigler M, Paul T, Grabitz RG (2005): Biocompatibility screening in cardiovascular implants. Z Kardiol 94:383-391.

Smith JD, Ogino H, Hunt D, Laylor RM, Rose ML, Yacoub MH (1995): Humoral immune response to human aortic valve homografts. Ann Thorac Surg 60:S127-S130.

Stanfill R, Nykanen DG, Osorio S, Whalen R, Burke RP, Zahn EM (2008): Stent implantation is effective treatment of vascular stenosis in young infants with congenital heart disease: acute implantation and longterm follow-up results. Catheter Cardiovasc Interv 71:831-841.

Stark J (1998): The use of valved conduits in pediatric cardiac surgery. Pediatr Cardiol 19:282-288.

Tamura K, Murakami M, Washizu M (2007): Healing of wound sutures on the mitral valve: an experimental study. Gen Thorac Cardiovasc Surg 55:98-104.

Tiete AR, Sachweh JS, Roemer U, Kozlik-Feldmann R, Reichart B, Daebritz SH (2004): Right ventricular outflow tract reconstruction with the Contegra bovine jugular vein conduit: a word of caution. Ann Thorac Surg $\underline{77}: 2151-2156$. 
Tweddell JS, Pelech AN, Frommelt PC, Mussatto KA, Wyman JD, Fedderly RT, Berger S, Frommelt MA, Lewis DA, Friedberg DZ, Thomas JP, Sachdeva R, Litwin SB (2000): Factors affecting longevity of homograft valves used in right ventricular outflow tract reconstruction for congenital heart disease. Circulation 102:III130-III135.

Valente M, Bortolotti U, Thiene G (1985): Ultrastructural substrates of dystrophic calcification in porcine bioprosthetic valve failure. Am J Pathol 119:12-21.

Valente M, Minarini M, Maizza AF, Bortolotti U, Thiene G (1992): Heart valve bioprosthesis durability: a challenge to the new generation of porcine valves. Eur J Cardiothorac Surg 6 Suppl 1:S82-S90.

Vivie de ER, Hellberg K, Kutzner DD, Rahlf G, Rupprath G, Beuren AJ (1981): Conduit repair for complex congenital heart disease with pulmonary atresia or right ventricular outflow tract obstruction. part I: surgical results. Thorac Cardiovasc Surg 29:329-336.

Vogt PR, Stallmach T, Niederhäuser U, Schneider J, Zünd G, Lachat M, Künzli A, Turina MI (1999): Explanted cryopreserved allografts: a morphological and immunohistochemical comparison between arterial allografts and allograft heart valves from infants and adults. Eur $J$ Cardiothorac Surg 15:639-44; discussion 644-5.

Webb CL, Nguyen NM, Schoen FJ, Levy RJ (1992): Calcification of allograft aortic wall in a rat subdermal model. Pathophysiology and inhibition by $\mathrm{Al} 3+$ and aminodiphosphonate preincubations. Am J Pathol 141:487-496.

Wilson W, Taubert KA, Gewitz M, Lockhart PB, Baddour LM, Levison M, Bolger A, Cabell CH, Takahashi M, Baltimore RS, Newburger JW, Strom BL, Tani LY, Gerber M, Bonow RO, Pallasch T, Shulman ST, Rowley AH, Burns JC, Ferrieri P, Gardner T, Goff D, Durack DT (2007): Prevention of infective endocarditis: guidelines from the American Heart Association: a guideline from the American Heart Association Rheumatic Fever, Endocarditis, and Kawasaki Disease Committee, Council on Cardiovascular Disease in the Young. Circulation 116:1736-1754.

Yankah AC, Alexi-Meskhishvili V,Weng Y, Berger F, Lange P, Hetzer R (1995a): Performance of aortic and pulmonary homografts in the right ventricular outflow tract in children. J Heart Valve Dis 4:392-395.

Yankah AC, Alexi-Meskhishvili V, Weng Y, Schorn K, Lange PE, Hetzer R (1995b): Accelerated degeneration of allografts in the first two years of life. Ann Thorac Surg 60:S71-6; discussion 576-7.

Yoshida T, Owens GK (2005): Molecular determinants of vascular smooth muscle cell diversity. Circ Res 96:280-291.

Yuan SM, Chang Q, Guo YR, Guo JQ (1998): In vitro pulsatile flow visualization on extracardiac conduits for the right ventricular outflow 
tract reconstruction: qualitative considerations. Kaohsiung J Med Sci $\underline{14}: 258-265$.

Zhang H, Williams GM (1992): Capillary and venule proliferation in the healing process of Dacron venous grafts in rats. Surgery 111:409-415. 